\title{
Carleman estimates for semi-discrete parabolic operators and application to the controllability of semi-linear semi-discrete parabolic equations
}

\author{
Franck Boyer ${ }^{\mathrm{a}}$, Jérôme Le Rousseau ${ }^{\mathrm{b}, *}$ \\ a Aix-Marseille Université, Laboratoire d'Analyse Topologie Probabilités (LATP), CNRS UMR 7353, 39 rue F. Joliot-Curie, \\ 13453 Marseille cedex 13, France \\ ${ }^{\mathrm{b}}$ Université d'Orléans, Laboratoire de Mathématiques - Analyse, Probabilités, Modélisation - Orléans (MAPMO), \\ CNRS UMR 7349, Fédération Denis Poisson, CNRS FR 2964, B.P. 6759, 45067 Orléans cedex 2, France
}

Received 22 August 2012; received in revised form 13 May 2013; accepted 29 July 2013

Available online 12 September 2013

\begin{abstract}
In arbitrary dimension, in the discrete setting of finite-differences we prove a Carleman estimate for a semi-discrete parabolic operator, in which the large parameter is connected to the mesh size. This estimate is applied for the derivation of a (relaxed) observability estimate, that yield some controlability results for semi-linear semi-discrete parabolic equations. Sub-linear and super-linear cases are considered.
\end{abstract}

(c) 2013 Elsevier Masson SAS. All rights reserved.

$M S C: 35 \mathrm{~K} 10 ; 35 \mathrm{~K} 58 ; 65 \mathrm{M} 06 ; 93 \mathrm{~B} 05 ; 93 \mathrm{~B} 07$

Keywords: Parabolic operator; Semi-discrete Carleman estimates; Observability; Null controllability; Semi-linear equations

\section{Introduction and notation}

Let $d \geqslant 1, L_{1}, \ldots, L_{d}$ be positive real numbers, and $\Omega=\prod_{1 \leqslant i \leqslant d}\left(0, L_{i}\right)$. We set $\boldsymbol{x}=\left(x_{1}, \ldots, x_{d}\right) \in \Omega$. With $\omega \Subset \Omega$ we consider the following parabolic problem in $(0, T) \times \Omega$, with $T>0$,

$$
\partial_{t} y-\nabla_{\boldsymbol{x}} \cdot\left(\Gamma \nabla_{\boldsymbol{x}} y\right)=\mathbf{1}_{\omega} v \quad \text { in }(0, T) \times \Omega, \quad y_{\mid \partial \Omega}=0, \quad \text { and } \quad y_{\mid t=0}=y_{0},
$$

where the diagonal diffusion tensor $\Gamma(\boldsymbol{x})=\operatorname{Diag}\left(\gamma_{1}(\boldsymbol{x}), \ldots, \gamma_{d}(\boldsymbol{x})\right)$ with $\gamma_{i}(\boldsymbol{x})>0$ satisfies

$$
\operatorname{reg}(\Gamma) \stackrel{\text { def }}{=} \operatorname{ess}_{\substack{\boldsymbol{x} \in \Omega \\ i=1, \ldots, d}}\left(\gamma_{i}(\boldsymbol{x})+\frac{1}{\gamma_{i}(\boldsymbol{x})}+\left|\nabla_{\boldsymbol{x}} \gamma_{i}(\boldsymbol{x})\right|\right)<+\infty
$$

\footnotetext{
* Corresponding author.

E-mail addresses: fboyer@latp.univ-mrs.fr (F. Boyer), jlr@univ-orleans.fr (J. Le Rousseau).
} 
The distributed null controllability problem consists in finding $v \in L^{2}((0, T) \times \Omega)$ such that $y(T)=0$. This problem was solved in the 90's by G. Lebeau and L. Robbiano [18] and A. Fursikov and O.Yu. Imanuvilov [12]. By a duality argument the null controllability result for (1.1) is equivalent to having the following observability inequality

$$
|q(0)|_{L^{2}(\Omega)}^{2} \leqslant C_{\mathrm{obs}}^{2}\|q\|_{L^{2}((0, T) \times \omega)}^{2},
$$

for any $q$ solution to $\left(\partial_{t}+\nabla_{\boldsymbol{x}} \cdot\left(\Gamma \nabla_{\boldsymbol{x}} y\right)\right) q=0$ and $q_{\mid \partial \Omega}=0$.

Let us consider the elliptic operator on $\Omega$ given by

$$
\mathcal{A}=-\nabla_{\boldsymbol{x}} \cdot\left(\Gamma \nabla_{\boldsymbol{x}}\right)=-\sum_{1 \leqslant i \leqslant d} \partial_{x_{i}}\left(\gamma_{i} \partial_{x_{i}}\right)
$$

with homogeneous Dirichlet boundary conditions on $\partial \Omega$. We shall introduce a finite-difference approximation of the operator $\mathcal{A}$. For a mesh $\mathfrak{M}$ that we shall describe below, associated with a discretization step $h$, the discrete operator will be denoted by $\mathcal{A}^{\mathfrak{M}}$. It will act on a finite dimensional space $\mathbb{R}^{\mathfrak{M}}$, of dimension $|\mathfrak{M}|$, and will be selfadjoint for the standard inner product in $\mathbb{R}^{\mathfrak{M}}$. Our main result is the derivation of a Carleman estimate for the operators $\partial_{t} \pm \mathcal{A}^{\mathfrak{M}}$, i.e., a weighted energy estimate with a localized observation term, which is uniform with respect to the discretization parameter $h$. The weight function is of exponential type.

There is a vast literature on Carleman estimates going back from the original work of T. Carleman [7] and the seminal work of L. Hörmander [13] (see also [14, Chapter 8] and [15, Chapter 28]). These estimates were first introduced for the purpose of proving and quantifying unique continuation (see [22] for manifold references). In more recent years, the field of applications of Carleman estimates has gone beyond the original domain they had been introduced for. They are also used in the study of inverse problems and control theory for PDEs. For an introduction to Carleman estimates and their applications to controllability of parabolic equations, as we shall use them here, we refer for instance to [9] and [17]. For applications of these estimates to inverse problems we refer to [20].

From the semi-discrete Carleman estimates we obtain, we deduce an observation inequality for the operator $\partial_{t}-\mathcal{A}^{\mathfrak{M}}+a$, where $a$ is a bounded potential function:

$$
\left|q_{h}(0)\right|_{L^{2}(\Omega)}^{2} \leqslant C_{\mathrm{obs}}^{2}\left(\left\|q_{h}\right\|_{L^{2}((0, T) \times \omega)}^{2}+e^{-C_{1} / h}\left|q_{h}(T)\right|_{L^{2}(\Omega)}^{2}\right),
$$

for $q_{h}$ (semi-discrete) solution to $\left(\partial_{t}-\mathcal{A}^{\mathfrak{M}}+a\right) q_{h}=0$. Special care is placed in the estimation of the observability constant $C_{\mathrm{obs}}$, in particular in its dependency upon $\|a\|_{\infty}$. It should be emphasized at this point that $C_{\mathrm{obs}}$ depends on the geometrical setting, i.e., on $\Omega, \omega$, the operator diffusion matrix $\Gamma$, and on the potential function $a$. Yet $C_{\mathrm{obs}}$ is not dependent on the discretization parameter $h$. The observability inequality (1.4) is weak as compared to that one can obtain in the continuous case; compare with (1.3). Here, there is an additional term in the right hand-side of the inequality. In fact, because of the presence of this term we shall speak of a relaxed observability inequality. Earlier work $[2,3]$ showed that this term cannot be removed and is connected to an obstruction to the null controllability of the semi-discrete problem in space dimension greater than two, as pointed out by a counter-example due to O. Kavian (see e.g. the review article [21]). Still, by duality, the relaxed observability estimate we derive is equivalent to a controllability result. Because of the aforementioned counter-example we do not exactly achieve null controllability at the discrete level, yet we reach a small target, which size goes to zero exponentially as the mesh size $h \rightarrow 0$. We speak of $h$-null controllability. This notion should not be confused with approximate controllability: the size of the neighborhood of zero reached by the solution of the parabolic equation at the final time $t=T$ is not fixed; it is a function of the discretization step. The study of relaxed observability estimates for dicretized parabolic equations was initiated by [19]. We refer to [6] for a review.

The dependency of the observability constant with respect to the norm $\|a\|_{\infty}$ allows one to tackle controllability questions for parabolic equations with semi-linear terms, in particular for some cases of super-linear terms. In the continuous case, this was achieved in [1,11]. To our knowledge, in the discrete case this question was only discussed in [10]. Here, we shall consider such questions in the case of semi-discretized equations and we shall be interested in proving $h$-null controllability results as well as associated relaxed observability inequalities. Some of the results we give are uniform with respect to the discretization parameter: $h$-null controllability is achieved with a (semi-discrete) control function whose $L^{2}$-norm is bounded uniformly in $h$. 


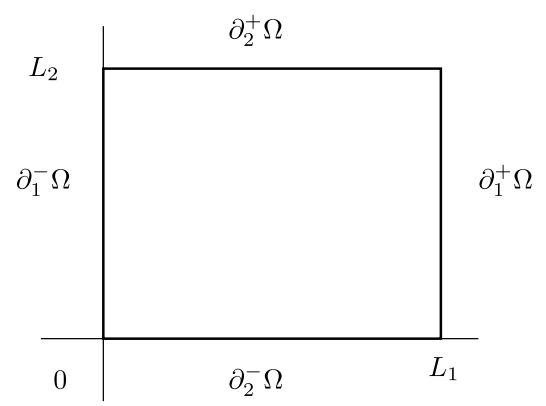

Fig. 1. Notation for the boundaries in the $2 \mathrm{D}$ case.

\subsection{Discrete settings and notation}

Precise statements of the results we obtain require the introduction of the settings we shall work with.

For $1 \leqslant i \leqslant d, i \in \mathbb{N}$, we set $\Omega_{i}=\prod_{\substack{1 \leqslant j \leqslant d \\ j \neq i}}\left(0, L_{j}\right)$. For $T>0$ we introduce

$$
Q=(0, T) \times \Omega, \quad Q_{i}=(0, T) \times \Omega_{i}, \quad 1 \leqslant i \leqslant d .
$$

We also set boundaries as (see Fig. 1)

$$
\begin{aligned}
& \partial_{i}^{-} \Omega=\prod_{1 \leqslant j<i}\left[0, L_{j}\right] \times\{0\} \times \prod_{i<j \leqslant d}\left[0, L_{j}\right], \quad \partial_{i}^{+} \Omega=\prod_{1 \leqslant j<i}\left[0, L_{j}\right] \times\left\{L_{i}\right\} \times \prod_{i<j \leqslant d}\left[0, L_{j}\right], \\
& \partial_{i} \Omega=\partial_{i}^{+} \Omega \cup \partial_{i}^{-} \Omega, \quad \partial \Omega=\bigcup_{1 \leqslant i \leqslant d} \partial_{i} \Omega .
\end{aligned}
$$

We shall use uniform meshes, i.e., meshes with constant discretization steps in each direction. The introduction of more general meshes is possible. We refer to [3] for some families of regular non-uniform meshes that one can consider.

The notation we introduce will allow us to use a formalism as close as possible to the continuous case, in particular for norms and integrations. Then most of the computations we carry out can be read in a very intuitive manner, which will ease the reading of the article. Most of the discrete formalism will then be hidden in the subsequent sections. The notation below is however necessary for a complete and precise reading of the proofs.

We shall use the notation $\llbracket a, b \rrbracket=[a, b] \cap \mathbb{N}$.

\subsubsection{Primal mesh}

For $i \in \llbracket 1, d \rrbracket$ and $N_{i} \in \mathbb{N}^{*}$, we set $h_{i}=L_{i} /\left(N_{i}+1\right)$ and $x_{i, j}=j h_{i}, j \in \llbracket 0, N_{i}+1 \rrbracket$, which gives

$$
0=x_{i, 0}<x_{i, 1}<\cdots<x_{i, N_{i}}<x_{i, N_{i}+1}=L_{i} .
$$

We introduce the following set of indices,

$$
\mathfrak{N}:=\left\{\boldsymbol{k}=\left(k_{1}, \ldots, k_{d}\right) ; k_{i} \in \llbracket 1, N_{i} \rrbracket, i \in \llbracket 1, d \rrbracket\right\} .
$$

For $\boldsymbol{k}=\left(k_{1}, \ldots, k_{d}\right) \in \mathfrak{N}$ we set $\boldsymbol{x}_{\boldsymbol{k}}=\left(x_{1, k_{1}}, \ldots, x_{d, k_{d}}\right) \in \Omega$. We refer to this discretization as to the primal mesh

$$
\mathfrak{M}:=\left\{\boldsymbol{x}_{\boldsymbol{k}} ; \boldsymbol{k} \in \mathfrak{N}\right\}, \quad \text { with }|\mathfrak{M}|:=\prod_{i \in \llbracket 1, d \rrbracket} N_{i} .
$$

We set $h=\max _{i \in \llbracket 1, d \rrbracket} h_{i}$ and we impose the following condition on the meshes that we consider: there exists $C>0$ such that

$$
C^{-1} h \leqslant h_{i} \leqslant C h, \quad i \in \llbracket 1, d \rrbracket .
$$




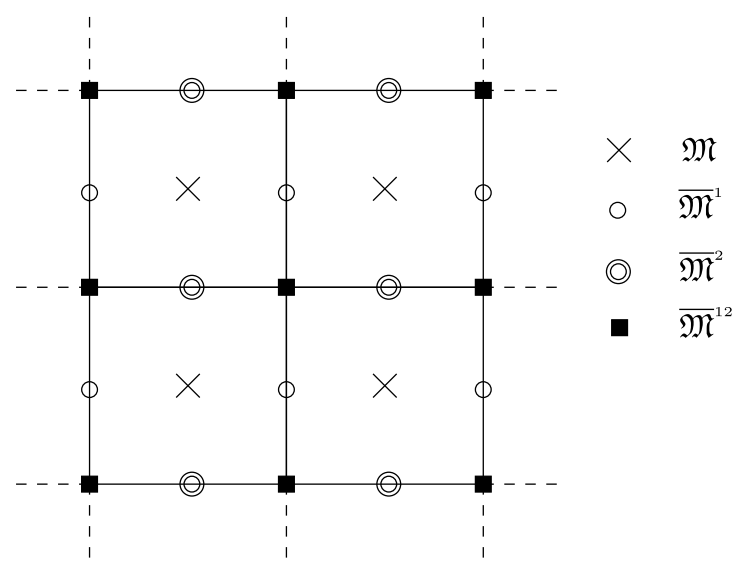

Fig. 2. Primal and dual meshes in the 2D case.

\subsubsection{Boundary of the primal mesh}

To introduce boundary conditions in the $i$ th direction and related trace operators (see Section 1.1.6) we set $\partial_{i} \mathfrak{N}=$ $\partial_{i}^{-} \mathfrak{N} \cup \partial_{i}^{+} \mathfrak{N}$ with

$$
\begin{aligned}
& \partial_{i}^{-} \mathfrak{N}=\left\{\boldsymbol{k}=\left(k_{1}, \ldots, k_{d}\right) ; k_{j} \in \llbracket 1, N_{j} \rrbracket, j \in \llbracket 1, d \rrbracket, j \neq i, k_{i}=0\right\}, \\
& \partial_{i}^{+} \mathfrak{N}=\left\{\boldsymbol{k}=\left(k_{1}, \ldots, k_{d}\right) ; k_{j} \in \llbracket 1, N_{j} \rrbracket, j \in \llbracket 1, d \rrbracket, j \neq i, k_{i}=N_{i}+1\right\},
\end{aligned}
$$

and

$$
\partial \mathfrak{N}=\bigcup_{i \in \llbracket 1, d \rrbracket} \partial_{i} \mathfrak{N}, \quad \partial \mathfrak{M}=\left\{\boldsymbol{x}_{\boldsymbol{k}} ; \boldsymbol{k} \in \partial \mathfrak{N}\right\}, \quad \partial_{i}^{ \pm} \mathfrak{M}=\left\{\boldsymbol{x}_{\boldsymbol{k}} ; \boldsymbol{k} \in \partial_{i}^{ \pm} \mathfrak{N}\right\}
$$

\subsubsection{Dual meshes}

We will need to operate discrete derivatives on functions defined on the primal mesh (see Section 1.1.5). It is easily seen that these derivatives are naturally associated to another set of staggered meshes, called dual meshes. In fact there will be two kinds of such meshes: the ones associated to a first-order discrete derivation and the ones associated to a second-order discrete derivation. Let us define precisely these new meshes (see Fig. 2).

For $i \in \llbracket 1, d \rrbracket$ and $N_{i} \in \mathbb{N}^{*}$, we set $x_{i, j}=j h_{i}$ for $j \in \llbracket 0, N_{i} \rrbracket+\frac{1}{2}$, which gives

$$
0=x_{i, 0}<x_{i, \frac{1}{2}}<x_{i, 1}<x_{i, 1+\frac{1}{2}}<\cdots<x_{i, N_{i}}<x_{i, N_{i}+\frac{1}{2}}<x_{i, N_{i}+1}=L_{i} .
$$

For $i \in \llbracket 1, d \rrbracket$, we introduce a second type of sets of indices

$$
\overline{\mathfrak{N}}^{i}:=\left\{\boldsymbol{k}=\left(k_{1}, \ldots, k_{d}\right) ; k_{j} \in \llbracket 1, N_{j} \rrbracket, j \in \llbracket 1, d \rrbracket, j \neq i \text {, and } k_{i} \in \llbracket 0, N_{i} \rrbracket+\frac{1}{2}\right\} .
$$

For $j \in \llbracket 1, d \rrbracket, j \neq i$, we also set $\partial_{j} \overline{\mathfrak{N}}^{i}=\partial_{j}^{-} \overline{\mathfrak{N}}^{i} \cup \partial_{j}^{+} \overline{\mathfrak{N}}^{i}$ with

$$
\begin{aligned}
& \partial_{j}^{-} \overline{\mathfrak{N}}^{i}=\left\{\boldsymbol{k}=\left(k_{1}, \ldots, k_{d}\right) ; k_{i^{\prime}} \in \llbracket 1, N_{i^{\prime}} \rrbracket, i^{\prime} \in \llbracket 1, d \rrbracket, i^{\prime} \neq i, i^{\prime} \neq j, k_{i} \in \llbracket 0, N_{i} \rrbracket+\frac{1}{2}, \text { and } k_{j}=0\right\}, \\
& \partial_{j}^{+} \overline{\mathfrak{N}}^{i}=\left\{\boldsymbol{k}=\left(k_{1}, \ldots, k_{d}\right) ; k_{i^{\prime}} \in \llbracket 1, N_{i^{\prime}} \rrbracket, i^{\prime} \in \llbracket 1, d \rrbracket, i^{\prime} \neq i, i^{\prime} \neq j, k_{i} \in \llbracket 0, N_{i} \rrbracket+\frac{1}{2}, \text { and } k_{j}=N_{j}+1\right\},
\end{aligned}
$$

and $\partial \overline{\mathfrak{N}}^{i}=\bigcup_{\substack{j \in \llbracket 1, d \rrbracket \\ j \neq i}} \partial_{j} \overline{\mathfrak{N}}^{i}$. We moreover introduce $\partial_{i} \overline{\mathfrak{N}}^{i}=\partial_{i}^{-} \overline{\mathfrak{N}}^{i} \cup \partial_{i}^{+} \overline{\mathfrak{N}}^{i}$ with

$$
\begin{aligned}
& \partial_{i}^{-} \overline{\mathfrak{N}}^{i}=\left\{\boldsymbol{k}=\left(k_{1}, \ldots, k_{d}\right) ; k_{j} \in \llbracket 1, N_{j} \rrbracket, j \in \llbracket 1, d \rrbracket, j \neq i, k_{i}=\frac{1}{2}\right\}, \\
& \partial_{i}^{+} \overline{\mathfrak{N}}^{i}=\left\{\boldsymbol{k}=\left(k_{1}, \ldots, k_{d}\right) ; k_{j} \in \llbracket 1, N_{j} \rrbracket, j \in \llbracket 1, d \rrbracket, j \neq i, k_{i}=N_{i}+\frac{1}{2}\right\} .
\end{aligned}
$$


Remark that $\partial_{i} \overline{\mathfrak{N}}^{i} \subset \overline{\mathfrak{N}}^{i}$ whereas $\partial_{j} \overline{\mathfrak{N}}^{i} \not \subset \overline{\mathfrak{N}}^{i}$ for $j \neq i$.

For $i, j \in \llbracket 1, d \rrbracket, i \neq j$, we introduce a third type of sets of indices

$$
\overline{\mathfrak{N}}^{i j}:=\left\{\boldsymbol{k}=\left(k_{1}, \ldots, k_{d}\right) ; k_{i^{\prime}} \in \llbracket 1, N_{i^{\prime}} \rrbracket, i^{\prime} \in \llbracket 1, d \rrbracket, i^{\prime} \neq i, i^{\prime} \neq j \text { and } k_{i} \in \llbracket 0, N_{i} \rrbracket+\frac{1}{2}, k_{j} \in \llbracket 0, N_{j} \rrbracket+\frac{1}{2}\right\} .
$$

For $l \in \llbracket 1, d \rrbracket, l \neq i, l \neq j$, we also set $\partial_{l} \overline{\mathfrak{N}}^{i j}=\partial_{l}^{-} \overline{\mathfrak{N}}^{i j} \cup \partial_{l}^{+} \overline{\mathfrak{N}}^{i j}$ with

$$
\begin{aligned}
\partial_{l}^{-} \overline{\mathfrak{N}}^{i j}= & \left\{\boldsymbol{k}=\left(k_{1}, \ldots, k_{d}\right) ; k_{i^{\prime}} \in \llbracket 1, N_{i^{\prime}} \rrbracket, i^{\prime} \in \llbracket 1, d \rrbracket, i^{\prime} \neq i, i^{\prime} \neq j, i^{\prime} \neq l,\right. \\
& \left.k_{i} \in \llbracket 0, N_{i} \rrbracket+\frac{1}{2}, k_{j} \in \llbracket 0, N_{j} \rrbracket+\frac{1}{2}, \text { and } k_{l}=0\right\}, \\
\partial_{l}^{+} \overline{\mathfrak{N}}^{i j}= & \left\{\boldsymbol{k}=\left(k_{1}, \ldots, k_{d}\right) ; k_{i^{\prime}} \in \llbracket 1, N_{i^{\prime}} \rrbracket, i^{\prime} \in \llbracket 1, d \rrbracket, i^{\prime} \neq i, i^{\prime} \neq j, i^{\prime} \neq l,\right. \\
& \left.k_{i} \in \llbracket 0, N_{i} \rrbracket+\frac{1}{2}, k_{j} \in \llbracket 0, N_{j} \rrbracket+\frac{1}{2}, \text { and } k_{l}=N_{l}+1\right\},
\end{aligned}
$$

and $\partial \overline{\mathfrak{N}}^{i j}=\bigcup_{\substack{l \in \llbracket 1, d \rrbracket \\ l \neq i, l \neq j}} \partial_{l} \overline{\mathfrak{N}}^{i j}$. Moreover we set $\partial_{i} \overline{\mathfrak{N}}^{i j}=\partial_{i}^{-} \overline{\mathfrak{N}}^{i j} \cup \partial_{i}^{+} \overline{\mathfrak{N}}^{i j}$ with

$$
\begin{aligned}
& \partial_{i}^{-} \overline{\mathfrak{N}}^{i j}=\left\{\boldsymbol{k}=\left(k_{1}, \ldots, k_{d}\right) ; k_{i^{\prime}} \in \llbracket 1, N_{i^{\prime}} \rrbracket, i^{\prime} \in \llbracket 1, d \rrbracket, i^{\prime} \neq i, i^{\prime} \neq j, k_{i}=\frac{1}{2}, k_{j} \in \llbracket 0, N_{j} \rrbracket+\frac{1}{2}\right\}, \\
& \partial_{i}^{+} \overline{\mathfrak{N}}^{i j}=\left\{\boldsymbol{k}=\left(k_{1}, \ldots, k_{d}\right) ; k_{i^{\prime}} \in \llbracket 1, N_{i^{\prime}} \rrbracket, i^{\prime} \in \llbracket 1, d \rrbracket, i^{\prime} \neq i, i^{\prime} \neq j, k_{i}=N_{i}+\frac{1}{2}, k_{j} \in \llbracket 0, N_{j} \rrbracket+\frac{1}{2}\right\} .
\end{aligned}
$$

For $\boldsymbol{k}=\left(k_{1}, \ldots, k_{d}\right) \in \overline{\mathfrak{N}}^{i}$ or $\partial \overline{\mathfrak{N}}^{i}$ (resp. $\overline{\mathfrak{N}}^{i j}$ or $\left.\partial \overline{\mathfrak{N}}^{i j}\right)$ we also set $\boldsymbol{x}_{\boldsymbol{k}}=\left(x_{1, k_{1}}, \ldots, x_{d, k_{d}}\right)$, which gives the following dual meshes

$$
\begin{aligned}
& \overline{\mathfrak{M}}^{i}:=\left\{\boldsymbol{x}_{\boldsymbol{k}} ; \boldsymbol{k} \in \overline{\mathfrak{N}}^{i}\right\}, \quad \partial \overline{\mathfrak{M}}^{i}:=\left\{\boldsymbol{x}_{\boldsymbol{k}} ; \boldsymbol{k} \in \partial \overline{\mathfrak{N}}^{i}\right\}, \quad \partial_{j}^{ \pm} \overline{\mathfrak{M}}^{i}:=\left\{\boldsymbol{x}_{\boldsymbol{k}} ; \boldsymbol{k} \in \partial_{j}^{ \pm} \overline{\mathfrak{N}}^{i}\right\}, \\
& \text { (resp. } \left.\overline{\mathfrak{M}}^{i j}:=\left\{\boldsymbol{x}_{\boldsymbol{k}} ; \boldsymbol{k} \in \overline{\mathfrak{N}}^{i j}\right\}, \partial \overline{\mathfrak{M}}^{i j}:=\left\{\boldsymbol{x}_{\boldsymbol{k}} ; \boldsymbol{k} \in \partial \overline{\mathfrak{N}}^{i j}\right\}, \partial_{l}^{ \pm} \overline{\mathfrak{M}}^{i j}:=\left\{\boldsymbol{x}_{\boldsymbol{k}} ; \boldsymbol{k} \in \partial_{l}^{ \pm} \overline{\mathfrak{N}}^{i j}\right\}\right) .
\end{aligned}
$$

\subsubsection{Discrete functions}

We denote by $\mathbb{R}^{\mathfrak{M}}$ (resp. $\mathbb{R}^{\overline{\mathfrak{M}}^{i}}$ or $\mathbb{R}^{\overline{\mathfrak{M}}^{i j}}$ ) the sets of discrete functions defined on $\mathfrak{M}$ (resp. $\overline{\mathfrak{M}}^{i}$ or $\overline{\mathfrak{M}}^{i j}$ ) respectively. If $u \in \mathbb{R}^{\mathfrak{M}}$ (resp. $\mathbb{R}^{\overline{\mathfrak{M}}^{i}}$ or $\mathbb{R}^{\bar{M}^{i j}}$ ), we denote by $u_{\boldsymbol{k}}$ its value corresponding to $\boldsymbol{x}_{\boldsymbol{k}}$ for $\boldsymbol{k} \in \mathfrak{N}$ (resp. $\boldsymbol{k} \in \overline{\mathfrak{N}}^{i}$ or $\boldsymbol{k} \in \overline{\mathfrak{N}}^{i j}$ ). For $u \in \mathbb{R}^{\mathfrak{M}}$ we define

$$
u^{\mathfrak{M}}=\sum_{\boldsymbol{k} \in \mathfrak{N}} \mathbf{1}_{b_{\boldsymbol{k}}} u_{\boldsymbol{k}} \in L^{\infty}(\Omega), \quad \text { with } b_{\boldsymbol{k}}=\prod_{i \in \llbracket 1, d \rrbracket}\left[x_{i, k_{i}-\frac{1}{2}}, x_{i, k_{i}+\frac{1}{2}}\right], \boldsymbol{k} \in \mathfrak{N} .
$$

Since no confusion is possible, by abuse of notation we shall often write $u$ in place of $u^{\mathfrak{M}}$. For $u \in \mathbb{R}^{\mathfrak{M}}$ we define

$$
\iint_{\Omega} u:=\iint_{\Omega} u^{\mathfrak{M}}(\boldsymbol{x}) d \boldsymbol{x}=\sum_{\boldsymbol{k} \in \mathfrak{N}}\left|b_{\boldsymbol{k}}\right| u_{\boldsymbol{k}}, \quad \text { where }\left|b_{\boldsymbol{k}}\right|=\prod_{i \in \llbracket 1, d \rrbracket} h_{i} .
$$

For some $u \in \mathbb{R}^{\mathfrak{M}}$, we shall need to associate boundary values

$$
u^{\partial \mathfrak{M}}=\left\{u_{\boldsymbol{k}} ; \boldsymbol{k} \in \partial \mathfrak{N}\right\},
$$

i.e., the values of $u$ at the point $\boldsymbol{x}_{\boldsymbol{k}} \in \partial \mathfrak{M}$. The set of such extended discrete functions is denoted by $\mathbb{R}^{\mathfrak{M} \cup \partial \mathfrak{M}}$. Homogeneous Dirichlet boundary conditions then consist in the choice $u_{\boldsymbol{k}}=0$ for $\boldsymbol{k} \in \partial \mathfrak{N}$, in short $u^{\partial \mathfrak{M}}=0$ or even $u_{\mid \partial \Omega}=0$ by abuse of notation (see also Section 1.1.6 below).

Similarly, for $u \in \mathbb{R}^{\overline{\mathfrak{M}}^{i}}$ (resp. $\mathbb{R}^{\overline{\mathfrak{M}}^{i j}}$ ) we shall associate the following boundary values

$$
u^{\partial \overline{\mathfrak{M}}^{i}}=\left\{u_{\boldsymbol{k}} ; \boldsymbol{k} \in \partial \overline{\mathfrak{N}}^{i}\right\} \quad\left(\text { resp. } u^{\partial \overline{\mathfrak{M}}^{i j}}=\left\{u_{\boldsymbol{k}} ; \boldsymbol{k} \in \partial \overline{\mathfrak{N}}^{i j}\right\}\right) .
$$

The set of such extended discrete functions is denoted by $\mathbb{R}^{\overline{\mathfrak{M}}^{i} \cup \partial \overline{\mathfrak{M}}^{i}}$ (resp. $\mathbb{R}^{\overline{\mathfrak{M}}^{i j} \cup \partial \overline{\mathfrak{M}}^{i j}}$ ). 
For $u \in \mathbb{R}^{\overline{\mathfrak{M}}^{i}}$ (resp. $\mathbb{R}^{\overline{\mathfrak{M}}^{i j}}$ ) we define

$$
\begin{aligned}
& u^{\overline{\mathfrak{M}}^{i}}=\sum_{\boldsymbol{k} \in \overline{\mathfrak{N}}^{i}} \mathbf{1}_{\bar{b}_{\boldsymbol{k}}^{i}} u_{\boldsymbol{k}} \in L^{\infty}(\Omega) \quad \text { with } \bar{b}_{\boldsymbol{k}}^{i}=\prod_{l \in \llbracket 1, d \rrbracket}\left[x_{l, k_{l}-\frac{1}{2}}, x_{l, k_{l}+\frac{1}{2}}\right], \boldsymbol{k} \in \overline{\mathfrak{N}}^{i}, \\
& \left(\text { resp. } u^{\overline{\mathfrak{M}}^{i j}}=\sum_{\boldsymbol{k} \in \overline{\mathfrak{N}}^{i j}} \mathbf{1}_{\bar{b}_{\boldsymbol{k}}^{i j}} u_{\boldsymbol{k}} \in L^{\infty}(\Omega) \quad \text { with } \bar{b}_{\boldsymbol{k}}^{i j}=\prod_{l \in \llbracket 1, d \rrbracket}\left[x_{l, k_{l}-\frac{1}{2}}, x_{l, k_{l}+\frac{1}{2}}\right], \boldsymbol{k} \in \overline{\mathfrak{N}}^{i j}\right) .
\end{aligned}
$$

As above, for $u \in \mathbb{R}^{\overline{\mathfrak{M}}^{i}}$ (resp. $\mathbb{R}^{\overline{\mathfrak{M}}^{i j}}$ ), we define

$$
\begin{aligned}
& \iint_{\Omega} u:=\iint_{\Omega} u^{\overline{\mathfrak{M}}^{i}}(\boldsymbol{x}) d \boldsymbol{x}=\sum_{\boldsymbol{k} \in \overline{\mathfrak{N}}^{i}}\left|\bar{b}_{\boldsymbol{k}}^{i}\right| u_{\boldsymbol{k}}, \quad \text { where }\left|\bar{b}_{\boldsymbol{k}}^{i}\right|=\prod_{l \in \llbracket 1, d \rrbracket} h_{l}, \\
& \text { (resp. } \left.\iint_{\Omega} u:=\iint_{\Omega} u^{i j}(\boldsymbol{x}) d \boldsymbol{x}=\sum_{\boldsymbol{k} \in \overline{\mathfrak{M}}^{i j}}\left|\bar{b}_{\boldsymbol{k}}^{i j}\right| u_{\boldsymbol{k}}, \quad \text { where }\left|\bar{b}_{\boldsymbol{k}}^{i j}\right|=\prod_{l \in \llbracket 1, d \rrbracket} h_{l}\right) .
\end{aligned}
$$

Remark 1.1. Above, the definitions of $b_{\boldsymbol{k}}, \bar{b}_{\boldsymbol{k}}^{i}$, and $\bar{b}_{\boldsymbol{k}}^{i j}$ look similar. They are however different as each time the multi-index $\boldsymbol{k}=\left(k_{1}, \ldots, k_{d}\right)$ is chosen in a different set: $\mathfrak{N}, \overline{\mathfrak{N}}^{i}$ and $\overline{\mathfrak{N}}^{i j}$ respectively.

In particular we define the following $L^{2}$-inner product on $\mathbb{R}^{\mathfrak{M}}$ (resp. $\mathbb{R}^{\overline{\mathfrak{M}}^{i}}$ or $\mathbb{R}^{\overline{\mathfrak{M}}^{i j}}$ )

$$
\begin{aligned}
& \langle u, v\rangle_{L^{2}(\Omega)}=\iint_{\Omega} u v=\iint_{\Omega} u^{\mathfrak{M}}(\boldsymbol{x}) v^{\mathfrak{M}}(\boldsymbol{x}) d \boldsymbol{x}, \\
& \left(\operatorname{resp} .\langle u, v\rangle_{L^{2}(\Omega)}=\iint_{\Omega} u v=\iint_{\Omega} u^{\overline{\mathfrak{M}}^{i}}(\boldsymbol{x}) v^{\overline{\mathfrak{M}}^{i}}(\boldsymbol{x}) d \boldsymbol{x},\right. \\
& \left.\quad \operatorname{or}\langle u, v\rangle_{L^{2}(\Omega)}=\iint_{\Omega} u v=\iint_{\Omega} u^{\overline{\mathfrak{M}}^{i j}}(\boldsymbol{x}) v^{\overline{\mathfrak{M}}^{i j}}(\boldsymbol{x}) d \boldsymbol{x}\right) .
\end{aligned}
$$

The associated norms will be denoted by $|u|_{L^{2}(\Omega)}$.

For semi-discrete function $u(t)$ in $\mathbb{R}^{\mathfrak{M}}$ (resp. $\mathbb{R}^{\overline{\mathfrak{M}}^{i}}$ or $\left.\mathbb{R}^{\overline{\mathfrak{M}}^{i j}}\right), t \in(0, T)$, we shall write $\iiint_{Q} u d t=\int_{0}^{T} \iint_{\Omega} u(t) d t$, and we define the following $L^{2}$-norm

$$
\|u(t)\|_{L^{2}(Q)}^{2}=\int_{0}^{T} \iint_{\Omega}(u(t))^{2} d t .
$$

Endowing the space of semi-discrete functions $L^{2}\left(0, T ; \mathbb{R}^{\mathfrak{M}}\right)\left(\right.$ resp. $L^{2}\left(0, T ; \mathbb{R}^{\overline{\mathfrak{M}}^{i}}\right)$ or $\left.L^{2}\left(0, T ; \mathbb{R}^{\overline{\mathfrak{M}}^{i j}}\right)\right)$ with this norm yields a Hilbert space.

Definition of a space of semi-discrete functions like $L^{\infty}\left(0, T, \mathbb{R}^{\mathfrak{M}}\right)\left(\right.$ resp. $L^{\infty}\left(0, T ; \mathbb{R}^{\overline{\mathfrak{M}}^{i}}\right)$ or $\left.L^{\infty}\left(0, T ; \mathbb{R}^{\overline{\mathfrak{M}}^{i j}}\right)\right)$ can be done similarly with the norm

$$
\|u(t)\|_{L^{\infty}(Q)}=\underset{t \in(0, T)}{\operatorname{ess} \sup }\left(\sup _{\boldsymbol{k} \in \mathfrak{N}}\left|u_{\boldsymbol{k}}(t)\right|\right) .
$$

We shall also use mixed norms of the form

$$
\|u(t)\|_{L^{\infty}\left(0, T ; L^{2}(\Omega)\right)}=\underset{t \in(0, T)}{\operatorname{ess} \sup }|u(t)|_{L^{2}(\Omega)} .
$$

Similarly we shall use such norms for spaces of semi-discrete functions defined on (or restricted to) $(0, T) \times \omega$. 


\subsubsection{Translation, difference and average operators}

Let $i, j \in \llbracket 1, d \rrbracket, j \neq i$. We define the following translations for indices:

$$
\begin{aligned}
\tau_{i}^{ \pm}: \overline{\mathfrak{N}}^{i}\left(\text { resp. } \overline{\mathfrak{N}}^{i j}\right) & \rightarrow \mathfrak{N} \cup \partial_{i}^{ \pm} \mathfrak{N}\left(\text { resp. } \overline{\mathfrak{N}}^{j} \cup \partial_{i}^{ \pm} \overline{\mathfrak{N}}^{j}\right), \\
\boldsymbol{k} & \mapsto \tau_{i}^{ \pm} \boldsymbol{k},
\end{aligned}
$$

with

$$
\left(\tau_{i}^{ \pm} \boldsymbol{k}\right)_{l}= \begin{cases}k_{l} & \text { if } l \neq i, \\ k_{l} \pm \frac{1}{2} & \text { if } l=i .\end{cases}
$$

Translations operators mapping $\mathbb{R}^{\mathfrak{M} \cup \partial \mathfrak{M}} \rightarrow \mathbb{R}^{\overline{\mathfrak{M}}^{i}}$ and $\mathbb{R}^{\overline{\mathfrak{M}}^{j} \cup \partial \overline{\mathfrak{M}}^{j}} \rightarrow \mathbb{R}^{\overline{\mathfrak{M}}^{i j}}$ are then given by

$$
\left(\tau_{i}^{ \pm} u\right)_{\boldsymbol{k}}=u_{\left(\tau_{i}^{ \pm} \boldsymbol{k}\right)}, \quad \boldsymbol{k} \in \overline{\mathfrak{N}}^{i}\left(\operatorname{resp} . \overline{\mathfrak{N}}^{i j}\right)
$$

A first-order difference operator $D_{i}$ and an averaging operator $A_{i}$ are then given by

$$
\begin{array}{ll}
\left(D_{i} u\right)_{k}=\left(h_{i}\right)^{-1}\left(\left(\tau_{i}^{+} u\right)_{k}-\left(\tau_{i}^{-} u\right)_{k}\right), & \boldsymbol{k} \in \overline{\mathfrak{N}}^{i}\left(\text { resp. } \overline{\mathfrak{N}}^{i j}\right), \\
\left(A_{i} u\right)_{k}=\tilde{u}_{\boldsymbol{k}}^{i}=\frac{1}{2}\left(\left(\tau_{i}^{+} u\right)_{\boldsymbol{k}}+\left(\tau_{i}^{-} u\right)_{k}\right), & \boldsymbol{k} \in \overline{\mathfrak{N}}^{i}\left(\text { resp. } \overline{\mathfrak{N}}^{i j}\right) .
\end{array}
$$

Both map $\mathbb{R}^{\mathfrak{M} \cup \partial \mathfrak{M}}$ into $\mathbb{R}^{\overline{\mathfrak{M}}^{i}}$ and $\mathbb{R}^{\overline{\mathfrak{M}}^{j} \cup \partial \overline{\mathfrak{M}}^{j}}$ into $\mathbb{R}^{\overline{\mathfrak{M}}^{i j}}$.

We also define the following translations for indices:

$$
\begin{aligned}
\bar{\tau}_{i}^{ \pm}: \mathfrak{N}\left(\text { resp. } \overline{\mathfrak{N}}^{j}\right) & \rightarrow \overline{\mathfrak{N}}^{i}\left(\operatorname{resp} . \overline{\mathfrak{N}}^{i j}\right), \\
\boldsymbol{k} & \mapsto \bar{\tau}_{i}^{ \pm} \boldsymbol{k},
\end{aligned}
$$

with

$$
\left(\bar{\tau}_{i}^{ \pm} \boldsymbol{k}\right)_{l}= \begin{cases}k_{l} & \text { if } l \neq i \\ k_{l} \pm \frac{1}{2} & \text { if } l=i\end{cases}
$$

Translations operators mapping $\mathbb{R}^{\overline{\mathfrak{M}}^{i}} \rightarrow \mathbb{R}^{\mathfrak{M}}$ and $\mathbb{R}^{\overline{\mathfrak{M}}^{i j}} \rightarrow \mathbb{R}^{\overline{\mathfrak{M}}^{j}}$ are then given by

$$
\left(\bar{\tau}_{i}^{ \pm} v\right)_{\boldsymbol{k}}=v_{\left(\bar{\tau}_{i}^{ \pm} \boldsymbol{k}\right)}, \quad \boldsymbol{k} \in \mathfrak{N}\left(\operatorname{resp} . \overline{\mathfrak{N}}^{j}\right) .
$$

A first-order difference operator $\bar{D}_{i}$ and an averaging operator $\bar{A}_{i}$ are then given by

$$
\begin{array}{ll}
\left(\bar{D}_{i} v\right)_{\boldsymbol{k}}=\left(h_{i}\right)^{-1}\left(\left(\bar{\tau}_{i}^{+} v\right)_{\boldsymbol{k}}-\left(\bar{\tau}_{i}^{-} v\right)_{\boldsymbol{k}}\right), & \boldsymbol{k} \in \mathfrak{N}\left(\operatorname{resp} . \overline{\mathfrak{N}}^{j}\right), \\
\left(\bar{A}_{i} v\right)_{\boldsymbol{k}}=\bar{v}_{\boldsymbol{k}}^{i}=\frac{1}{2}\left(\left(\bar{\tau}_{i}^{+} v\right)_{\boldsymbol{k}}+\left(\bar{\tau}_{i}^{-} v\right)_{\boldsymbol{k}}\right), & \boldsymbol{k} \in \mathfrak{N}\left(\operatorname{resp} . \overline{\mathfrak{N}}^{j}\right) .
\end{array}
$$

Both map $\mathbb{R}^{\overline{\mathfrak{M}}^{i}}$ into $\mathbb{R}^{\mathfrak{M}}$ and $\mathbb{R}^{\overline{\mathfrak{M}}^{i j}}$ into $\mathbb{R}^{\overline{\mathfrak{M}}^{j}}$.

\subsubsection{Traces}

Let $i \in \llbracket 1, d \rrbracket$. For $u \in \mathbb{R}^{\mathfrak{M} \cup \partial \mathfrak{M}}$ (resp. $\mathbb{R}^{\overline{\mathfrak{M}}^{j} \cup \partial \overline{\mathfrak{M}}^{j}}, j \neq i$ ), its trace on $\partial_{i}^{+} \Omega$, corresponds to $\boldsymbol{k} \in \partial_{i}^{+} \mathfrak{N}$ (resp. $\partial_{i}^{+} \overline{\mathfrak{N}}^{j}$ ), i.e., $k_{i}=N_{i}+1$ in our discretization and will be denoted by $u_{\mid k_{i}=N_{i}+1}$ or simply $u_{N_{i}+1}$. Similarly its trace on $\partial_{i}^{-} \Omega$, corresponds to $\boldsymbol{k} \in \partial_{i}^{-} \mathfrak{N}$ (resp. $\partial_{i}^{-} \overline{\mathfrak{N}}^{j}$ ), i.e., $k_{i}=0$ and will be denoted by $u_{\mid k_{i}=0}$ or simply $u_{0}$. The latter notation will be used if no confusion is possible, that is if the context indicates that the trace is taken on $\partial_{i}^{-} \Omega$.

By abuse of notation, we shall also use $\partial_{i} \Omega, i \in \llbracket 1, d \rrbracket$, to denote the boundaries of $\Omega$ in the discrete setting. For homogeneous Dirichlet boundary condition we shall write

$$
\begin{aligned}
v_{\mid \partial \Omega}=0 & \Leftrightarrow \quad v_{\mid \partial_{i} \Omega}=0, i \in \llbracket 1, d \rrbracket \\
& \Leftrightarrow \quad v_{\mid k_{i}=0}=v_{\mid k_{i}=N_{i}+1}=0, i \in \llbracket 1, d \rrbracket .
\end{aligned}
$$


We set

$$
\mathbb{R}^{\mathfrak{M} \cup \partial \mathfrak{M}}{ }_{0}=\left\{v \in \mathbb{R}^{\mathfrak{M} \cup \partial \mathfrak{M}} ; v_{\mid k_{i}=0}=v_{\mid k_{i}=N_{i}+1}=0, i \in \llbracket 1, d \rrbracket\right\} .
$$

For $v \in \mathbb{R}^{\overline{\mathfrak{M}}^{i} \cup \partial \overline{\mathfrak{M}}^{i}}$ (resp. $\mathbb{R}^{\overline{\mathfrak{M}}^{i j} \cup \partial \overline{\mathfrak{M}}^{i j}}, j \neq i$ ), its trace on $\partial_{i}^{+} \Omega$, corresponds to $\boldsymbol{k} \in \partial_{i}^{+} \overline{\mathfrak{N}}^{i}$ (resp. $\partial_{i}^{+} \overline{\mathfrak{N}}^{i j}$ ), i.e., $k_{i}=$ $N_{i}+\frac{1}{2}$ in our discretization and will be denoted by $v_{\mid k_{i}=N_{i}+\frac{1}{2}}$ or simply $v_{N_{i}+\frac{1}{2}}$. Similarly its trace on $\partial_{i}^{-} \Omega$, corresponds to $\boldsymbol{k} \in \partial_{i}^{-} \overline{\mathfrak{N}}^{i}$ (resp. $\partial_{i}^{-} \overline{\mathfrak{N}}^{i j}$ ), i.e., $k_{i}=\frac{1}{2}$ and will be denoted by $v_{\mid k_{i}=\frac{1}{2}}$ or simply $v_{\frac{1}{2}}$. The latter notation will be used if no confusion is possible, if the context indicates that the trace is taken on $\partial_{i}^{-} \Omega$.

For such functions $u \in \mathbb{R}^{\mathfrak{M} \cup \partial \mathfrak{M}}$ (resp. $\mathbb{R}^{\overline{\mathfrak{M}}^{j} \cup \partial \overline{\mathfrak{M}}^{j}}, j \neq i$ ) we can then define surface integrals of the type

$$
\int_{\partial_{i}^{+} \Omega} u_{\mid \partial_{i}^{+} \Omega}=\int_{\Omega_{i}} u_{\mid k_{i}=N_{i}+1}=\sum_{\substack{\left.\boldsymbol{k} \in \partial_{i}^{+} \mathfrak{N} \\ \text { (resp. } \boldsymbol{k} \in \partial_{i}^{+} \overline{\mathfrak{N}}^{j}\right)}}\left|\partial_{i} b_{\boldsymbol{k}}\right| u_{\boldsymbol{k}}, \quad \text { where }\left|\partial_{i} b_{\boldsymbol{k}}\right|=\prod_{\substack{l \in \llbracket 1, d \rrbracket \\ l \neq i}} h_{l}, \boldsymbol{k} \in \partial_{i}^{+} \mathfrak{N}\left(\text { resp. } \partial_{i}^{+} \overline{\mathfrak{N}}^{j}\right),
$$

and for $v \in \mathbb{R}^{\overline{\mathfrak{M}}^{i} \cup \partial \overline{\mathfrak{M}}^{i}}$ (resp. $\mathbb{R}^{\overline{\mathfrak{M}}^{i j} \cup \partial \overline{\mathfrak{M}}^{i j}}, j \neq i$ )

$$
\int_{\partial_{i}^{+} \Omega} v_{\mid \partial_{i}^{+} \Omega}=\int_{\Omega_{i}} v_{\mid k_{i}=N_{i}+\frac{1}{2}}=\sum_{\substack{\left.\boldsymbol{k} \in \partial_{i}^{+} \overline{\mathfrak{N}}^{i} \\ \text { (resp. } \boldsymbol{k} \in \partial_{i}^{+} \overline{\mathfrak{N}}^{i j}\right)}}\left|\partial_{i} \bar{b}_{\boldsymbol{k}}^{i}\right| v_{\boldsymbol{k}}, \quad \text { where }\left|\partial_{i} \bar{b}_{\boldsymbol{k}}^{i}\right|=\prod_{\substack{l \in \llbracket 1, d \rrbracket \\ l \neq i}} h_{l}, \boldsymbol{k} \in \partial_{i}^{+} \overline{\mathfrak{N}}^{i}\left(\text { resp. } \partial_{i}^{+} \overline{\mathfrak{N}}^{i j}\right) .
$$

Observe that if $\boldsymbol{k} \in \partial_{i}^{+} \mathfrak{N}$ (resp. $\partial_{i}^{+} \overline{\mathfrak{N}}^{j}$ ) and $\boldsymbol{k}^{\prime} \in \partial_{i}^{+} \overline{\mathfrak{N}}^{i}$ (resp. $\partial_{i}^{+} \overline{\mathfrak{N}}^{i j}$ ) with $k_{l}=k_{l}^{\prime}$ for $l \neq i$ then $\left|\partial_{i} b_{\boldsymbol{k}}\right|=\mid \partial_{i} \bar{b}_{\boldsymbol{k}^{\prime}}^{i}$. We thus have

$$
\int_{\partial_{i}^{+} \Omega} v_{\mid \partial_{i}^{+} \Omega}=\int_{\Omega_{i}} v_{\mid k_{i}=N_{i}+\frac{1}{2}}=\int_{\Omega_{i}}\left(\bar{\tau}_{i}^{-} v\right)_{\mid k_{i}=N_{i}+1}=\int_{\partial_{i}^{+} \Omega}\left(\bar{\tau}_{i}^{-} v\right)_{\mid \partial_{i}^{+} \Omega}
$$

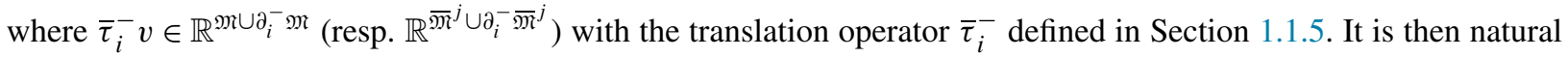
to define the following integrals

$$
\int_{\Omega_{i}} u_{N_{i}+1} v_{N_{i}+\frac{1}{2}}=\int_{\Omega_{i}} u_{\mid k_{i}=N_{i}+1} v_{\mid k_{i}=N_{i}+\frac{1}{2}}=\int_{\Omega_{i}}\left(u \bar{\tau}_{i}^{-} v\right)_{\mid k_{i}=N_{i}+1}=\int_{\partial_{i}^{+} \Omega} u\left(\bar{\tau}_{i}^{-} v\right)_{\mid \partial_{i}^{+} \Omega} .
$$

Such trace integrals will appear when applying discrete integrations by parts in the following sections.

Similar definitions and considerations can be made for integrals over $\partial_{i}^{-} \Omega$.

For $u \in \mathbb{R}^{\mathfrak{M} \cup \partial \mathfrak{M}}$ (resp. $\mathbb{R}^{\overline{\mathfrak{M}}^{j} \cup \partial \overline{\mathfrak{M}}^{j}}, j \neq i$ ) we can then introduce the following $L^{2}$-norm for the trace on $\partial_{i} \Omega$ :

$$
|u|_{L^{2}\left(\partial_{i} \Omega\right)}^{2}=\left|u_{\mid \partial_{i} \Omega}\right|_{L^{2}\left(\partial_{i} \Omega\right)}^{2}=\int_{\Omega_{i}}\left(u_{\mid k_{i}=N_{i}+1}\right)^{2}+\int_{\Omega_{i}}\left(u_{\mid k_{i}=0}\right)^{2} .
$$

For $v \in \mathbb{R}^{\overline{\mathfrak{M}}^{i} \cup \partial \overline{\mathfrak{M}}^{i}}$ (resp. $\mathbb{R}^{\overline{\mathfrak{M}}^{i j} \cup \partial \overline{\mathfrak{M}}^{i j}}, j \neq i$ ) we can then introduce the following $L^{2}$-norm for the trace on $\partial_{i} \Omega$ :

$$
|v|_{L^{2}\left(\partial_{i} \Omega\right)}^{2}=\left|v_{\mid \partial_{i} \Omega}\right|_{L^{2}\left(\partial_{i} \Omega\right)}^{2}=\int_{\Omega_{i}}\left(u_{\mid k_{i}=N_{i}+\frac{1}{2}}\right)^{2}+\int_{\Omega_{i}}\left(u_{\mid k_{i}=\frac{1}{2}}\right)^{2} .
$$

\subsubsection{Sampling of continuous functions}

A continuous function $f$ defined on $\bar{\Omega}$ can be sampled on the primal mesh $f^{\mathfrak{M}}=\left\{f\left(\boldsymbol{x}_{\boldsymbol{k}}\right) ; \boldsymbol{k} \in \mathfrak{N}\right\}$, which we identify to

$$
f^{\mathfrak{M}}=\sum_{k \in \mathfrak{N}} \mathbf{1}_{b_{k}} f_{k}, \quad f_{k}=f\left(x_{k}\right), k \in \mathfrak{N}
$$


with $b_{\boldsymbol{k}}$ as defined in (1.6). We also set

$$
f^{\partial \mathfrak{M}}=\left\{f\left(\boldsymbol{x}_{\boldsymbol{k}}\right) ; \boldsymbol{k} \in \partial \mathfrak{N}\right\}, \quad f^{\mathfrak{M} \cup \partial \mathfrak{M}}=\left\{f\left(\boldsymbol{x}_{\boldsymbol{k}}\right) ; \boldsymbol{k} \in \mathfrak{N} \cup \partial \mathfrak{N}\right\} .
$$

The function $f$ can also be sampled on the dual meshes, e.g. $\overline{\mathfrak{M}}^{i}, f^{i}=\left\{f\left(\boldsymbol{x}_{\boldsymbol{k}}\right) ; \boldsymbol{k} \in \overline{\mathfrak{M}}^{i}\right\}$ which we identify to

$$
f^{\overline{\mathfrak{M}}^{i}}=\sum_{\boldsymbol{k} \in \overline{\mathfrak{N}}^{i}} \mathbf{1}_{\bar{b}_{\boldsymbol{k}}^{i}} f_{\boldsymbol{k}}, \quad f_{\boldsymbol{k}}=f\left(\boldsymbol{x}_{\boldsymbol{k}}\right), \boldsymbol{k} \in \overline{\mathfrak{N}}^{i}
$$

with similar definitions for $f^{\partial \overline{\mathfrak{M}}^{i}}, f^{\overline{\mathfrak{M}}^{i} \cup \partial \overline{\mathfrak{M}}^{i}}$ and sampling on the meshes $\overline{\mathfrak{M}}^{i j}, \overline{\mathfrak{M}}^{i j} \cup \partial \overline{\mathfrak{M}}^{i j}$.

In the sequel, we shall use the symbol $f$ for both the continuous function and its sampling on the primal or dual meshes. In fact, from the context, one will be able to deduce the appropriate sampling. For example, with $u$ defined on the primal mesh, $\mathfrak{M}$, in the following expression, $\bar{D}_{i}\left(\gamma D_{i} u\right)$, it is clear that the function $\gamma$ is sampled on the dual mesh $\overline{\mathfrak{M}}^{i}$ as $D_{i} u$ is defined on this mesh and the operator $\bar{D}_{i}$ acts on functions defined on this mesh.

To evaluate the action of multiple iterations of discrete operators, e.g. $D_{i}, \bar{D}_{i}, A_{i}, \bar{A}_{i}$ on a continuous function we may require the function to be defined in a neighborhood of $\bar{\Omega}$. This will be the case here of the diffusion coefficients in the elliptic operator and the Carleman weight function we shall introduce. For a function $f$ defined on a neighborhood of $\bar{\Omega}$ we set

$$
\begin{aligned}
& \tau_{i}^{ \pm} f(\boldsymbol{x}):=f\left(\boldsymbol{x} \pm \frac{h_{i}}{2} \boldsymbol{e}_{i}\right), \quad \boldsymbol{e}_{i}=\left(\delta_{i 1}, \ldots, \delta_{i d}\right), \\
& \mathrm{D}_{i} f:=\left(h_{i}\right)^{-1}\left(\tau_{i}^{+}-\tau_{i}^{-}\right) f, \quad \mathrm{~A}_{i} f=\hat{f}^{i}=\frac{1}{2}\left(\tau_{i}^{+}+\tau_{i}^{-}\right) f .
\end{aligned}
$$

For a function $f$ continuously defined in a neighborhood of $\bar{\Omega}$, the discrete function $D_{i} f$ is in fact equal to $D_{i} f$ sampled on the dual mesh, $\overline{\mathfrak{M}}^{i}$, and $\bar{D}_{i} f$ is equal to $\mathrm{D}_{i} f$ sampled on the primal mesh, $\mathfrak{M}$. We shall use similar meanings for averaging symbols, $\tilde{f}, \bar{f}$, and for more general combinations: for instance, if $i \neq j, \widetilde{D_{j}} f^{i},{\overline{D_{i}} \bar{D}_{j} f}^{i}$, ${\overline{D_{i} D_{j} f}}^{i}$ will be respectively the functions ${\widehat{\mathrm{D}_{j} f}}^{i}$ sampled on $\overline{\mathfrak{M}}^{i j},{\widehat{\mathrm{D}_{i} \mathrm{D}_{j} f}}^{i}$ sampled on $\mathfrak{M}$, and ${\widehat{\mathrm{D}_{i} \mathrm{D}_{j} f}}^{i}$ sampled on $\overline{\mathfrak{M}}^{j}$.

\subsection{Statement of the main results}

With the notation we have introduced, the usual consistent finite-difference approximation of the elliptic operator $\mathcal{A}$ with homogeneous Dirichlet boundary conditions is

$$
\mathcal{A}^{\mathfrak{M}} u=-\sum_{i \in \llbracket 1, d \rrbracket} \bar{D}_{i}\left(\gamma_{i} D_{i} u\right)
$$

for $u \in \mathbb{R}^{\mathfrak{M} \cup \partial \mathfrak{M}}$ satisfying $u_{\mid \partial \Omega}=u^{\partial \mathfrak{M}}=0$. Recall that, in each term, $\gamma_{i}$ is the sampling of the given continuous diffusion coefficient $\gamma_{i}$ on the dual mesh $\overline{\mathfrak{M}}^{i}$, so that for any $u \in \mathbb{R}^{\mathfrak{M} \cup \partial \mathfrak{M}}$ and $\boldsymbol{k} \in \mathfrak{N}$, we have

$$
\left(\mathcal{A}^{\mathfrak{M}} u\right)_{(\boldsymbol{k})}=-\sum_{i \in \llbracket 1, d \rrbracket} h_{i}^{-2}\left(\gamma_{i}\left(\boldsymbol{x}_{\bar{\tau}_{i}^{+}(\boldsymbol{k})}\right)\left(\left(\bar{\tau}_{i}^{+} \tau_{i}^{+} u\right)_{(\boldsymbol{k})}-u_{(\boldsymbol{k})}\right)-\gamma_{i}\left(\boldsymbol{x}_{\bar{\tau}_{i}^{-}(\boldsymbol{k})}\right)\left(u_{(\boldsymbol{k})}-\left(\bar{\tau}_{i}^{-} \tau_{i}^{-} u\right)_{(\boldsymbol{k})}\right)\right) \text {. }
$$

In $2 \mathrm{D}$, this operator is nothing but the standard 5-point discretization. Note however that other consistent choices of discretization of $\gamma_{i}$ on the dual meshes are possible, such as the averaging on the dual mesh $\overline{\mathfrak{M}}^{i}$ of the sampling of $\gamma_{i}$ on the primal mesh.

The semi-discrete forward and backward parabolic operators are then given by $P_{ \pm}^{\mathfrak{M}}=\partial_{t} \pm \mathcal{A}^{\mathfrak{M}}$.

\subsubsection{Carleman estimate}

For the Carleman estimate and observation and control results we choose here to treat the case of a distributed observation in $\omega \Subset \Omega$. The weight function is of the form $r=e^{s \varphi}$ with $\varphi=e^{\lambda \psi}$, with $\psi$ fulfilling the following assumption. Construction of such a weight function is classical (see e.g. [12]). 
Assumption 1.2. Let $\omega_{0} \Subset \omega$ be an open set. Let $\tilde{\Omega}$ be a smooth open and connected neighborhood of $\bar{\Omega}$ in $\mathbb{R}^{d}$. The function $\boldsymbol{x} \mapsto \psi(\boldsymbol{x})$ is in $\mathscr{C}^{p}(\overline{\tilde{\Omega}}, \mathbb{R}), p$ sufficiently large, and satisfies, for some $c>0$,

$$
\psi>0 \quad \text { in } \tilde{\Omega}, \quad|\nabla \psi| \geqslant c \quad \text { in } \tilde{\Omega} \backslash \omega_{0}, \quad \text { and } \quad \partial_{n_{i}} \psi(x) \leqslant-c<0, \quad \text { for } \boldsymbol{x} \in V_{\partial_{i}} \Omega,
$$

where $V_{\partial_{i}} \Omega$ is a sufficiently small neighborhood of $\partial_{i} \Omega$ in $\tilde{\Omega}$, in which the outward unit normal $n_{i}$ to $\Omega$ is extended from $\partial_{i} \Omega$.

\section{Remark 1.3.}

1. In the continuous case the regularity of the weight function can be chosen as low as $\mathscr{C}^{2}$. Here estimates of discrete derivations of the weight function require knowledge of higher-order derivatives through Taylor formulae.

2. In [3] the following addtional requirement was made: $\partial_{x_{i}}^{2} \psi(\boldsymbol{x}) \geqslant 0$ in $V_{\partial_{i}} \Omega$. This additional assumption is in fact not needed. This property was solely used in the proof of Lemma C.4 within the proof of Lemma 3.3 of [3]. Yet estimate (C.7) therein simply follows from the earlier estimates in Lemma C.4 without the need of this addtional property. We shall invoke the result of Lemma 3.3 of [3] here.

Let $K>\|\psi\|_{\infty}$ and set

$$
\begin{aligned}
& \varphi(\boldsymbol{x})=e^{\lambda \psi(\boldsymbol{x})}-e^{\lambda K}<0, \quad \phi(\boldsymbol{x})=e^{\lambda \psi(\boldsymbol{x})}, \\
& r(t, \boldsymbol{x})=e^{s(t) \varphi(\boldsymbol{x})}, \quad \rho(t, \boldsymbol{x})=(r(t, \boldsymbol{x}))^{-1},
\end{aligned}
$$

with

$$
s(t)=\tau \theta(t), \quad \tau>0, \theta(t)=((t+\delta T)(T+\delta T-t))^{-1},
$$

for $0<\delta<\frac{1}{2}$. The parameter $\delta$ is introduced to avoid singularities at time $t=0$ and $t=T$. Further comments are provided in Remark 1.5 below.

We now state our first result, a uniform Carleman estimate for the semi-discrete parabolic operators $P_{ \pm}^{\mathfrak{M}}=\partial_{t} \pm \mathcal{A}^{\mathfrak{M}}$.

Theorem 1.4. Let $\mathrm{reg}^{0}>0$ be given and let a function $\psi$ satisfy Assumption 1.2. We then define the function $\varphi$ according to (1.9). For the parameter $\lambda \geqslant 1$ sufficiently large, there exist $C, \tau_{0} \geqslant 1, h_{0}>0, \varepsilon_{0}>0$, depending on $\omega$, $\omega_{0}, T, \mathrm{reg}^{0}$, and $\lambda$, such that for any $\Gamma$, with $\operatorname{reg}(\Gamma) \leqslant \operatorname{reg}^{0}$ we have

$$
\begin{aligned}
\tau^{-1}\left\|\theta^{-\frac{1}{2}} e^{\tau \theta \varphi} \partial_{t} u\right\|_{L^{2}(Q)}^{2}+\tau \sum_{i \in \llbracket 1, d \rrbracket}\left(\left\|\theta e^{\tau \theta \varphi} D_{i} u\right\|_{L^{2}(Q)}^{2}+\left\|\theta e^{\tau \theta \varphi}{\overline{D_{i} u}}^{i}\right\|_{L^{2}(Q)}^{2}\right)+\tau^{3}\left\|\theta^{\frac{3}{2}} e^{\tau \theta \varphi} u\right\|_{L^{2}(Q)}^{2} \\
\leqslant C\left(\left\|e^{\tau \theta \varphi} P_{ \pm}^{\mathfrak{M}} u\right\|_{L^{2}(Q)}^{2}+\tau^{3}\left\|\theta^{\frac{3}{2}} e^{\tau \theta \varphi} u\right\|_{L^{2}((0, T) \times())}^{2}\right) \\
\quad+C h^{-2}\left(\left|e^{\tau \theta \varphi} u_{\mid t=0}\right|_{L^{2}(\Omega)}^{2}+\left|e^{\tau \theta \varphi} u_{\mid t=T}\right|_{L^{2}(\Omega)}^{2}\right),
\end{aligned}
$$

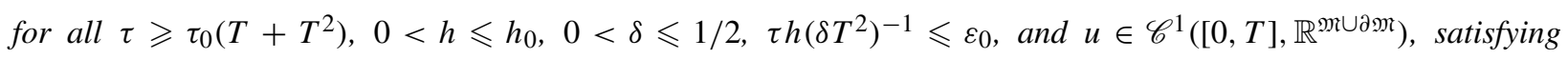
$u_{\mid(0, T) \times \partial \Omega}=0$.

Remark 1.5 (Choice of the parameter $\delta$ ). In the present Carleman estimate the parameter $\delta$ is introduced to avoid the singularity of the weight function at times $t=0$ and $t=T$. Such singularities, corresponding to the case $\delta=0$, are exploited in the continuous case as originally introduced in [12]. Here the parameter $\delta$ is taken different from 0 and yet connected to the other parameters: $\tau h\left(\delta T^{2}\right)^{-1} \leqslant \varepsilon_{0}$. Many choices are possible for $\delta$. For the controllability results we shall choose $\delta$ proportional to the discretization parameter $h$.

Remark 1.6. It should be emphasized that the geometrical domains considered here are rectangular parallelepipeds and moreover the diffussion matrix is assumed diagonal. This is very much related to the discretization with finite differences that allows us to use discrete differential calculus results, as exposed in Section 2. 
Remark 1.7. An inspection of the proof of Theorem 1.4 in Section 3 shows that for $P_{-}^{\mathfrak{M}}$ the term

$$
h^{-2}\left(\left|e^{\tau \theta \varphi} u_{\mid t=0}\right|_{L^{2}(\Omega)}^{2}+\left|e^{\tau \theta \varphi} u_{\mid t=T}\right|_{L^{2}(\Omega)}^{2}\right)
$$

in the r.h.s. of estimate (1.10) can be replaced by

$$
\tau^{2}\left(\left|e^{\tau \theta \varphi} \theta u_{\mid t=0}\right|_{L^{2}(\Omega)}^{2}+\left|e^{\tau \theta \varphi} \theta u_{\mid t=T}\right|_{L^{2}(\Omega)}^{2}\right)+\sum_{i \in \llbracket 1, d \rrbracket}\left|e^{\tau \theta \varphi} D_{i} u_{\mid t=T}\right|_{L^{2}(\Omega)}^{2} .
$$

\subsubsection{Relaxed observability estimate}

The adjoint system associated with the controlled system with potential

$$
\partial_{t} y+\mathcal{A}^{\mathfrak{M}} y+a y=\mathbf{1}_{\omega} v, \quad t \in(0, T), \quad y_{\mid \partial \Omega}=0,
$$

is given by

$$
-\partial_{t} q+\mathcal{A}^{\mathfrak{M}} q+a q=0, \quad t \in(0, T), \quad q_{\mid \partial \Omega}=0 .
$$

With the Carleman estimate we proved in Theorem 1.4 we have the following relaxed observability estimate for the solutions to (1.12):

$$
|q(0)|_{L^{2}(\Omega)}^{2} \leqslant C_{\mathrm{obs}}^{2}\left(\|q\|_{L^{2}((0, T) \times \omega)}^{2}+e^{-\frac{C_{1}}{h}}|q(T)|_{L^{2}(\Omega)}^{2}\right),
$$

with $C_{\mathrm{obs}}=e^{C_{2}\left(1+\frac{1}{T}+T\|a\|_{\infty}+\|a\|_{\infty}^{\frac{2}{3}}\right)}$, if the discretization parameter is chosen sufficiently small. A precise statement and a proof are given in Section 4.1. Note that $C_{\mathrm{obs}}$ is not function of the discretization parameter $h$.

\subsubsection{Controllability results}

From the relaxed observability estimate given above we obtain a $h$-null controllability result for the linear operator $P_{-}^{\mathfrak{M}}$. This result can be extended to classes of semi-linear equations:

$$
\left(\partial_{t}+\mathcal{A}^{\mathfrak{M}}\right) y+\mathcal{G}(y)=\mathbf{1}_{\omega} v, \quad t \in(0, T), \quad y_{\mid \partial \Omega}=0, \quad y(0)=y_{0},
$$

with $\mathcal{G}(y)=y g(y)$. The equation is linearized yielding a bounded potential and a control can be built. Then a fixedpoint argument allows one to obtain a control function for the non-linear equation.

First we consider the sub-linear case, i.e., we assume that $g$ is bounded. We then prove a $h$-null controllability result with a control that satisfies

$$
\|v\|_{L^{2}(Q)} \leqslant C\left|y_{0}\right|_{L^{2}(\Omega)},
$$

were the constant $C$ is uniform with respect to the discretization parameter $h$; see Section 5.1 for a precise statement and a proof.

Second we consider classes of super-linear equations. Following [11] we assume that we have

$$
|g(y)| \leqslant K \ln ^{r}(e+|y|), \quad y \in \mathbb{R}, \quad \text { with } 0 \leqslant r<\frac{3}{2} .
$$

Here the precise dependency of the observability constant upon the norm of the potential $\|a\|_{\infty}$ allows one to tackle such nonlinearities.

In arbitrary dimension we obtain a $h$-null controllability result; see Section 5.2.2 for a precise statement and a proof. However, the size of the control function is not proven uniform with respect to the discretization parameter $h$ :

$$
\|v\|_{L^{2}(Q)} \leqslant C_{h}\left|y_{0}\right|_{L^{2}(\Omega)} .
$$

In fact a boundedness argument is needed and here we exploit the finite dimensional structure to achieve it. The constants are however not uniform. A refined treatment of this question require further analysis of the semi-discrete heat kernel; see Remark 5.6. In one space dimension, this difficulty can be circumvented and the uniformity of the control function is recovered; see Section 5.2.3 


\subsection{Outline}

In Section 2 we present discrete calculus results and estimates for the Carleman weight function in preparation for the proof of Theorem 1.4. Section 3 is devoted to the proof of Theorem 1.4. In Section 4 we prove the relaxed observability estimate and a $h$-null controllability results in the linear case. In Section 5 we study $h$-null controllability in the semi-linear case. Some technical proofs are gathered in Appendix A.

\section{Some preliminary discrete calculus results}

This section aims to provide calculus rules for discrete operators such as $D_{i}, \bar{D}_{i}$ and also to provide estimates for the successive applications of such operators on the weight functions.

\subsection{Discrete calculus formulae}

Proofs are similar to that given in the one dimension case in [2].

Lemma 2.1. Let the functions $f_{1}$ and $f_{2}$ be continuously defined in a neighborhood of $\bar{\Omega}$. For $i \in \llbracket 1, d \rrbracket$, we have

$$
\mathrm{D}_{i}\left(f_{1} f_{2}\right)=\mathrm{D}_{i}\left(f_{1}\right) \hat{f}_{2}^{i}+\hat{f}_{1}^{i} \mathrm{D}_{i}\left(f_{2}\right) .
$$

Note that the immediate translation of the proposition to discrete functions $f_{1}, f_{2} \in \mathbb{R}^{\mathfrak{M}}$ (resp. $\mathbb{R}^{\overline{\mathfrak{M}}^{j}}, j \neq i$ ), and $g_{1}, g_{2} \in \mathbb{R}^{\overline{\mathfrak{M}}^{i}}$ (resp. $\mathbb{R}^{\overline{\mathfrak{M}}^{i j}}, j \neq i$ )

$$
D_{i}\left(f_{1} f_{2}\right)=D_{i}\left(f_{1}\right) \tilde{f}_{2}^{i}+\tilde{f}_{1}^{i} D_{i}\left(f_{2}\right), \quad \bar{D}_{i}\left(g_{1} g_{2}\right)=\bar{D}_{i}\left(g_{1}\right) \bar{g}_{2}^{i}+\bar{g}_{1}^{i} \bar{D}_{i}\left(g_{2}\right) .
$$

Lemma 2.2. Let the functions $f_{1}$ and $f_{2}$ be continuously defined in a neighborhood of $\bar{\Omega}$. For $i \in \llbracket 1, d \rrbracket$, we have

$$
{\widehat{f_{1} f_{2}}}^{i}=\hat{f}_{1}^{i} \hat{f}_{2}^{i}+\frac{h_{i}^{2}}{4} \mathrm{D}_{i}\left(f_{1}\right) \mathrm{D}_{i}\left(f_{2}\right) \text {. }
$$

Note that the immediate translation of the proposition to discrete functions $f_{1}, f_{2} \in \mathbb{R}^{\mathfrak{M}}$ (resp. $\mathbb{R}^{\overline{\mathfrak{M}}^{j}}, j \neq i$ ), and $g_{1}, g_{2} \in \mathbb{R}^{\overline{\mathfrak{M}}^{i}}$ (resp. $\mathbb{R}^{\overline{\mathfrak{M}}^{i j}}, j \neq i$ )

$$
{\widetilde{f_{1} f_{2}}}^{i}=\tilde{f}_{1}^{i} \tilde{f}_{2}^{i}+\frac{h_{i}^{2}}{4} D_{i}\left(f_{1}\right) D_{i}\left(f_{2}\right), \quad{\overline{g_{1} g_{2}}}^{i}=\bar{g}_{1}^{i} \bar{g}_{2}^{i}+\frac{h_{i}^{2}}{4} \bar{D}_{i}\left(g_{1}\right) \bar{D}_{i}\left(g_{2}\right)
$$

Some of the following properties can be extended in such a manner to discrete functions. We shall not always write it explicitly.

Averaging a function twice gives the following formula.

Lemma 2.3. Let the function $f$ be continuously defined over $\mathbb{R}$. For $i \in \llbracket 1, d \rrbracket$ we have

$$
\mathrm{A}_{i}^{2} f:=\widehat{\hat{f}}^{i}=f+\frac{h_{i}^{2}}{4} \mathrm{D}_{i} \mathrm{D}_{i} f
$$

The following proposition covers discrete integrations by parts and related formulae.

Proposition 2.4. Let $f \in \mathbb{R}^{\mathfrak{M} \cup 2 \mathfrak{M}}$ and $g \in \mathbb{R}^{\overline{\mathfrak{M}}^{i}}$. For $i \in \llbracket 1, d \rrbracket$ we have

$$
\begin{aligned}
& \iint_{\Omega} f\left(\bar{D}_{i} g\right)=-\iint_{\Omega}\left(D_{i} f\right) g+\int_{\Omega_{i}}\left(f_{N_{i}+1} g_{N_{i}+\frac{1}{2}}-f_{0} g_{\frac{1}{2}}\right), \\
& \iint_{\Omega} f \bar{g}^{i}=\iint_{\Omega} \tilde{f}^{i} g-\frac{h_{i}}{2} \int_{\Omega_{i}}\left(f_{N_{i}+1} g_{N_{i}+\frac{1}{2}}+f_{0} g_{\frac{1}{2}}\right) .
\end{aligned}
$$


Lemma 2.5. Let $i \in \llbracket 1, d \rrbracket$ and $v \in \mathbb{R}^{\mathfrak{M} \cup \partial \mathfrak{M}}$ (resp. $\mathbb{R}^{\overline{\mathfrak{M}}^{j} \cup \partial \overline{\mathfrak{M}}^{j}}$ for $j \neq i$ ) be such that $v_{\mid \partial_{i} \Omega}=0$. Then $\iint_{\Omega} v=\iint_{\Omega} \tilde{v}^{i}$.

Lemma 2.6. Let $f$ be a smooth function defined in a neighborhood of $\bar{\Omega}$. For $i \in \llbracket 1, d \rrbracket$ we have

$$
\begin{aligned}
& \tau_{i}^{ \pm} f=f \pm \frac{h_{i}}{2} \int_{0}^{1} \partial_{i} f\left(. \pm \sigma \boldsymbol{h}_{i} / 2\right) d \sigma, \quad \mathrm{A}_{i}^{\ell} f=f+C_{\ell} h_{i}^{2} \int_{-1}^{1}(1-|\sigma|) \partial_{i}^{2} f\left(.+l_{\ell} \sigma \boldsymbol{h}_{i}\right) d \sigma \\
& \mathrm{D}_{i}^{\ell} f=\partial_{i}^{\ell} f+C_{\ell}^{\prime} h_{i}^{2} \int_{-1}^{1}(1-|\sigma|)^{\ell+1} \partial_{i}^{\ell+2} f\left(.+l_{\ell} \sigma \boldsymbol{h}_{i}\right) d \sigma, \quad \ell=1,2, l_{1}=\frac{1}{2}, l_{2}=1,
\end{aligned}
$$

with $\boldsymbol{h}_{i}=h_{i} \boldsymbol{e}_{i}$.

For $i, j \in \llbracket 1, d \rrbracket, i \neq j$, we have

$$
\begin{aligned}
\mathrm{D}_{i} \mathrm{D}_{j} f= & \partial_{i j}^{2} f+C^{\prime \prime} \frac{\left|\boldsymbol{h}_{i j}^{+}\right|^{4}}{h_{i} h_{j}} \int_{-1}^{1}(1-|\sigma|)^{3} f^{(4)}\left(.+\sigma \boldsymbol{h}_{i j}^{+} / 2 ; \boldsymbol{\eta}^{+}, \ldots, \boldsymbol{\eta}^{+}\right) d \sigma \\
& +C^{\prime \prime \prime} \frac{\left|\boldsymbol{h}_{i j}^{+}\right|^{4}}{h_{i} h_{j}} \int_{-1}^{1}(1-|\sigma|)^{3} f^{(4)}\left(\boldsymbol{x}+\sigma \boldsymbol{h}_{i j}^{-} / 2 ; \boldsymbol{\eta}^{-}, \ldots, \boldsymbol{\eta}^{-}\right) d \sigma,
\end{aligned}
$$

with $\boldsymbol{h}_{i j}^{ \pm}=h_{i} \boldsymbol{e}_{i} \pm h_{j} \boldsymbol{e}_{j}$ and $\boldsymbol{\eta}^{ \pm}=\frac{1}{\left|\boldsymbol{h}_{i j}^{ \pm}\right|}\left(\boldsymbol{h}_{i j}^{ \pm}\right)$

Note that $\frac{\left|\boldsymbol{h}_{i j}^{+}\right|^{4}}{h_{i} h_{j}}=\mathcal{O}\left(h^{2}\right)$ by (1.5), for $i, j \in \llbracket 1, d \rrbracket, j \neq i$.

\subsection{Calculus results related to the weight functions}

We now present some technical lemmata related to discrete operations performed on the Carleman weight functions $\rho$ and $r=\rho^{-1}$, as defined in Section 1.2.1. The positive parameters $\tau$ and $h$ will be large and small respectively and we are particularly interested in the dependence on $\tau, h$ and $\lambda$ in the following basic estimates.

We assume $\tau \geqslant 1$ and $\lambda \geqslant 1$.

Lemma 2.7. Let $\alpha$ and $\beta$ be multi-indices in the $\boldsymbol{x}$ variable. We have

$$
\begin{aligned}
\partial^{\beta}\left(r \partial^{\alpha} \rho\right) & =|\alpha|^{|\beta|}(-s \phi)^{|\alpha|} \lambda^{|\alpha+\beta|}(\nabla \psi)^{\alpha+\beta}+|\alpha||\beta|(s \phi)^{|\alpha|} \lambda^{|\alpha+\beta|-1} \mathcal{O}(1)+s^{|\alpha|-1}|\alpha|(|\alpha|-1) \mathcal{O}_{\lambda}(1) \\
& =\mathcal{O}_{\lambda}\left(s^{|\alpha|}\right) .
\end{aligned}
$$

Let $\sigma \in[-1,1]$ and $i \in \llbracket 1, d \rrbracket$. We have

$$
\partial^{\beta}\left(r(t, .)\left(\partial^{\alpha} \rho\right)\left(t, .+\sigma \boldsymbol{h}_{i}\right)\right)=\mathcal{O}_{\lambda}\left(s^{|\alpha|}\left(1+(s h)^{|\beta|}\right)\right) e^{\mathcal{O}_{\lambda}(s h)} .
$$

Provided $\tau h\left(\max _{[0, T]} \theta\right) \leqslant \mathfrak{K}$ we have $\partial^{\beta}\left(r(t,).\left(\partial^{\alpha} \rho\right)\left(t, .+\sigma \boldsymbol{h}_{i}\right)\right)=\mathcal{O}_{\lambda, \mathfrak{K}}\left(s^{|\alpha|}\right)$. The same expressions hold with $r$ and $\rho$ interchanged and with s changed into $-s$.

A proof is given in [2, proof of Lemma 3.7] in the time independent case. This proof applies to the time-dependent case by noting that the condition $\tau h\left(\max _{[0, T]} \theta\right) \leqslant \mathfrak{K}$ implies that $s(t) h \leqslant \mathfrak{K}$ for all $t \in[0, T]$.

Lemma 2.8. Let $\alpha$ be a multi-index in the $\boldsymbol{x}$ variable. We have

$$
\partial_{t}\left(r \partial^{\alpha} \rho\right)=s^{|\alpha|} T \theta \mathcal{O}_{\lambda}(1) .
$$


Proof. We proceed by induction on $|\alpha|$. The result holds for $|\alpha|=0$, and we assume it also holds in the case $|\alpha|=n$. In the case $|\alpha|=n+1$, with $|\alpha| \geqslant 1$, we write $\alpha=\alpha^{\prime}+\alpha^{\prime \prime}$ with $\left|\alpha^{\prime \prime}\right|=1$ and we have

$$
r \partial^{\alpha} \rho=-s r \partial^{\alpha^{\prime}}\left(\left(\partial^{\alpha^{\prime \prime}} \varphi\right) \rho\right)=-\left(\sum_{\delta^{\prime}+\delta^{\prime \prime}=\alpha^{\prime}}\left(\begin{array}{l}
\alpha^{\prime} \\
\delta^{\prime}
\end{array}\right)\left(\partial^{\delta^{\prime \prime}+\alpha^{\prime \prime}} \varphi\right) s r \partial^{\delta^{\prime}} \rho\right) \text {. }
$$

Next we write

$$
\left|\partial_{t}\left(s r \partial^{\delta^{\prime}} \rho\right)\right| \leqslant\left|\left(\partial_{t} s\right) r \partial^{\delta^{\prime}} \rho\right|+\left|s \partial_{t}\left(r \partial^{\delta^{\prime}} \rho\right)\right| \leqslant s T \theta s^{\left|\delta^{\prime}\right|}
$$

by (3.1) and Lemma 2.7 for the estimation of the first term and by the inductive hypothesis for the second term. We then conclude as $\left|\delta^{\prime}\right|+1 \leqslant\left|\alpha^{\prime}\right|+1=|\alpha|=n+1$.

With the Leibniz formula we have the following estimate.

Corollary 2.9. Let $\alpha, \alpha^{\prime}$, and $\beta$ be multi-indices in the $\boldsymbol{x}$ variable. We have

$$
\begin{aligned}
\partial^{\beta}\left(r^{2}\left(\partial^{\alpha} \rho\right) \partial^{\alpha^{\prime}} \rho\right)= & \left|\alpha+\alpha^{\prime}\right|^{|\beta|}(-s \phi)^{\left|\alpha+\alpha^{\prime}\right|} \lambda^{\left|\alpha+\alpha^{\prime}+\beta\right|}(\nabla \psi)^{\alpha+\alpha^{\prime}+\beta} \\
& +|\beta|\left|\alpha+\alpha^{\prime}\right|(s \phi)^{\left|\alpha+\alpha^{\prime}\right|} \lambda^{\left|\alpha+\alpha^{\prime}+\beta\right|-1} \mathcal{O}(1) \\
& +s^{\left|\alpha+\alpha^{\prime}\right|-1}\left(|\alpha|(|\alpha|-1)+\left|\alpha^{\prime}\right|\left(\left|\alpha^{\prime}\right|-1\right)\right) \mathcal{O}_{\lambda}(1) \\
= & \mathcal{O}_{\lambda}\left(s^{\left|\alpha+\alpha^{\prime}\right|}\right) .
\end{aligned}
$$

The proofs of the following properties can be found in Appendix A of [3] (except that of Proposition 2.14 which is specific to the parabolic case).

Proposition 2.10. Let $\alpha$ be a multi-index in the $\boldsymbol{x}$ variable. Let $i, j \in \llbracket 1, d \rrbracket$, provided $\tau$ h $\max _{[0, T]} \theta \leqslant \mathfrak{K}$, we have

$$
\begin{aligned}
& r \tau_{i}^{ \pm} \partial^{\alpha} \rho=r \partial^{\alpha} \rho+s^{|\alpha|} \mathcal{O}_{\lambda, \mathfrak{K}}(s h)=s^{|\alpha|} \mathcal{O}_{\lambda, \mathfrak{K}}(1), \\
& r \mathrm{~A}_{i}{ }^{k} \partial^{\alpha} \rho=r \partial^{\alpha} \rho+s^{|\alpha|} \mathcal{O}_{\lambda, \mathfrak{K}}\left((s h)^{2}\right)=s^{|\alpha|} \mathcal{O}_{\lambda, \mathfrak{K}}(1), \quad k=1,2, \\
& r \mathrm{~A}_{i}{ }^{k} \mathrm{D}_{i} \rho=r \partial_{x} \rho+s \mathcal{O}_{\lambda, \mathfrak{K}}\left((s h)^{2}\right)=s \mathcal{O}_{\lambda, \mathfrak{K}}(1), \quad k=0,1, \\
& r \mathrm{D}_{i}{ }^{k_{i}} \mathrm{D}_{j}{ }^{k_{j}} \rho=r \partial_{i}^{k_{i}} \partial_{j}^{k_{j}} \rho+s^{2} \mathcal{O}_{\lambda, \mathfrak{K}}\left((s h)^{2}\right)=s^{2} \mathcal{O}_{\lambda, \mathfrak{K}}(1), \quad k_{i}+k_{j} \leqslant 2 .
\end{aligned}
$$

The same estimates hold with $\rho$ and $r$ interchanged.

Lemma 2.11. Let $\alpha$ and $\beta$ be multi-indices in the $\boldsymbol{x}$ variable and $k \in \mathbb{N}$. Let $i, j \in \llbracket 1, d \rrbracket$, provided $\tau$ h $\max _{[0, T]} \theta \leqslant \mathfrak{K}$, we have

$$
\begin{aligned}
& \mathrm{D}_{i}{ }^{k_{i}} \mathrm{D}_{j}{ }^{k_{j}}\left(\partial^{\beta}\left(r \partial^{\alpha} \rho\right)\right)=\partial_{i}^{k_{i}} \partial_{j}^{k_{j}} \partial^{\beta}\left(r \partial^{\alpha} \rho\right)+h^{2} \mathcal{O}_{\lambda, \mathfrak{K}}\left(s^{|\alpha|}\right), \quad k_{i}+k_{j} \leqslant 2, \\
& \mathrm{~A}_{i}{ }^{k} \partial^{\beta}\left(r \partial^{\alpha} \rho\right)=\partial^{\beta}\left(r \partial^{\alpha} \rho\right)+h^{2} \mathcal{O}_{\lambda, \mathfrak{K}}\left(s^{|\alpha|}\right) .
\end{aligned}
$$

Let $\sigma \in[-1,1]$, we have $\mathrm{D}_{i} k_{i} \mathrm{D}_{j} k_{j} \partial^{\beta}\left(r(t,.) \partial^{\alpha} \rho\left(t, .+\sigma \boldsymbol{h}_{i}\right)\right)=\mathcal{O}_{\lambda, \mathfrak{K}}\left(s^{|\alpha|}\right)$, for $k_{i}+k_{j} \leqslant 2$. The same estimates hold with $r$ and $\rho$ interchanged.

Lemma 2.12. Let $\alpha, \alpha^{\prime}$ and $\beta$ be multi-indices in the $\boldsymbol{x}$ variable and $k \in \mathbb{N}$. Let $i, j \in \llbracket 1, d \rrbracket$, provided $\tau$ h $\max _{[0, T]} \theta \leqslant$ $\mathfrak{K}$, we have

$$
\begin{gathered}
\mathrm{A}_{i}{ }^{k} \partial^{\beta}\left(r^{2}\left(\partial^{\alpha} \rho\right) \partial^{\alpha^{\prime}} \rho\right)=\partial^{\beta}\left(r^{2}\left(\partial^{\alpha} \rho\right) \partial^{\alpha^{\prime}} \rho\right)+h^{2} \mathcal{O}_{\lambda, \mathfrak{K}}\left(s^{|\alpha|+\left|\alpha^{\prime}\right|}\right)=\mathcal{O}_{\lambda, \mathfrak{K}}\left(s^{|\alpha|+\left|\alpha^{\prime}\right|}\right), \\
\mathrm{D}_{i}{ }^{k_{i}} \mathrm{D}_{j}{ }^{k_{j}} \partial^{\beta}\left(r^{2}\left(\partial^{\alpha} \rho\right) \partial^{\alpha^{\prime}} \rho\right) \\
=\partial_{i}^{k_{i}} \partial_{j}^{k_{j}}\left(\partial^{\beta}\left(r^{2}\left(\partial^{\alpha} \rho\right) \partial^{\alpha^{\prime}} \rho\right)\right)+h^{2} \mathcal{O}_{\lambda, \mathfrak{K}}\left(s^{|\alpha|+\left|\alpha^{\prime}\right|}\right) \\
=\mathcal{O}_{\lambda, \mathfrak{K}}\left(s^{|\alpha|+\left|\alpha^{\prime}\right|}\right), \quad k_{i}+k_{j} \leqslant 2 .
\end{gathered}
$$


Let $\sigma, \sigma^{\prime} \in[-1,1]$. We have

$$
\begin{aligned}
& \mathrm{A}_{i}{ }^{k} \partial^{\beta}\left(r(t, .)^{2}\left(\partial^{\alpha} \rho\left(t, .+\sigma \boldsymbol{h}_{i}\right)\right) \partial^{\alpha^{\prime}} \rho\left(t, .+\sigma^{\prime} \boldsymbol{h}_{j}\right)\right)=\mathcal{O}_{\lambda, \mathfrak{K}}\left(s^{|\alpha|+\left|\alpha^{\prime}\right|}\right), \\
& \mathrm{D}_{i}{ }^{k_{i}} \mathrm{D}_{j} k_{j} \partial^{\beta}\left(r(t, .)^{2}\left(\partial^{\alpha} \rho\left(t, .+\sigma \boldsymbol{h}_{i}\right)\right) \partial^{\alpha^{\prime}} \rho\left(t, .+\sigma^{\prime} \boldsymbol{h}_{j}\right)\right)=\mathcal{O}_{\lambda, \mathfrak{K}}\left(s^{|\alpha|+\left|\alpha^{\prime}\right|}\right), \quad k_{i}+k_{j} \leqslant 2 .
\end{aligned}
$$

The same estimates hold with $r$ and $\rho$ interchanged.

Proposition 2.13. Let $\alpha$ be a multi-index in the $\boldsymbol{x}$ variable and $k \in \mathbb{N}$. Let $i, j \in \llbracket 1, d \rrbracket$, provided $s h \leqslant \mathfrak{K}$, we have

$$
\begin{aligned}
& \mathrm{D}_{i}^{k_{i}} \mathrm{D}_{j}{ }^{k_{j}} \mathrm{~A}_{i}{ }^{k} \partial^{\alpha}\left(r{\widehat{\mathrm{D}_{i} \rho}}^{i}\right)=\partial_{i}^{k_{i}} \partial_{j}^{k_{j}} \partial^{\alpha}\left(r \partial_{x} \rho\right)+s \mathcal{O}_{\lambda, \mathfrak{K}}\left((s h)^{2}\right)=s \mathcal{O}_{\lambda, \mathfrak{K}}(1), \\
& \mathrm{D}_{i}^{k_{i}} \mathrm{D}_{j}{ }^{k}{ }_{j}\left(r \mathrm{D}_{i}^{2} \rho\right)=\partial_{i}^{k_{i}} \partial_{j}^{k_{j}}\left(r \partial_{i}^{2} \rho\right)+s^{2} \mathcal{O}_{\lambda, \mathfrak{K}}\left((s h)^{2}\right)=s^{2} \mathcal{O}_{\lambda, \mathfrak{K}}(1), \\
& r \mathrm{~A}_{i}^{2} \rho=1+\mathcal{O}_{\lambda, \mathfrak{K}}\left((s h)^{2}\right), \quad \mathrm{D}_{i}^{k_{i}} \mathrm{D}_{j}{ }^{k_{j}}\left(r \mathrm{~A}_{i}{ }^{2} \rho\right)=\mathcal{O}_{\lambda, \mathfrak{K}}\left((s h)^{2}\right) .
\end{aligned}
$$

The same estimates hold with $r$ and $\rho$ interchanged.

Proposition 2.14. Provided $\tau h \max _{[0, T]} \theta \leqslant \mathfrak{K}$, and $\sigma$ is bounded, we have

$$
\begin{aligned}
& \partial_{t}\left(r(., \boldsymbol{x})\left(\partial^{\alpha} \rho\right)\left(., \boldsymbol{x}+\sigma \boldsymbol{h}_{i}\right)\right)=T s^{|\alpha|} \theta(t) \mathcal{O}_{\lambda, \mathfrak{K}}(1), \\
& \partial_{t}\left(r \mathrm{~A}_{i}^{2} \rho\right)=T(s h)^{2} \theta(t) \mathcal{O}_{\lambda, \mathfrak{K}}(1), \\
& \partial_{t}\left(r \mathrm{D}_{i}^{2} \rho\right)=T s^{2} \theta(t) \mathcal{O}_{\lambda, \mathfrak{K}}(1) .
\end{aligned}
$$

The same estimates hold with $r$ and $\rho$ interchanged.

Proof. We set $v\left(t, \boldsymbol{x}, \sigma h_{i}\right):=r(t, \boldsymbol{x}) \rho\left(t, \boldsymbol{x}+\sigma \boldsymbol{h}_{i}\right)$ and simply have $v\left(t, \boldsymbol{x}, \sigma h_{i}\right)=e^{s(t)\left(\varphi(\boldsymbol{x})-\varphi\left(\boldsymbol{x}+\sigma \boldsymbol{h}_{i}\right)\right)}=e^{\mathcal{O}_{\lambda}(s(t) h)}=$ $\mathcal{O}_{\lambda, \mathfrak{K}}(1)$, by a first-order Taylor formula. We have

$$
\partial_{t} v\left(t, \boldsymbol{x}, \sigma h_{i}\right)=\left(\partial_{t} s\right)\left(\varphi(\boldsymbol{x})-\varphi\left(\boldsymbol{x}+\sigma \boldsymbol{h}_{i}\right)\right) v\left(t, \boldsymbol{x}, \sigma h_{i}\right)=\tau \theta^{\prime}(t) h_{i} \mathcal{O}_{\lambda, \mathfrak{K}}(1)=T \theta(t) \mathcal{O}_{\lambda, \mathfrak{K}}(s(t) h),
$$

by (3.1).

Next, we write $r(t, \boldsymbol{x})\left(\partial^{\alpha} \rho\right)\left(t, \boldsymbol{x}+\sigma \boldsymbol{h}_{i}\right)=v\left(t, \boldsymbol{x}, \sigma h_{i}\right) \mu_{\alpha}\left(t, \boldsymbol{x}+\sigma \boldsymbol{h}_{i}\right)$, where we have set $\mu_{\alpha}=r \partial_{i}^{\alpha} \rho$. We have

$$
\partial_{t} \mu_{\alpha}=T s^{|\alpha|} \theta \mathcal{O}_{\lambda, \mathfrak{K}}(1),
$$

by Lemma 2.8. This yields

$$
\begin{aligned}
\partial_{t}\left(r(t, \boldsymbol{x})\left(\partial^{\alpha} \rho\right)\left(t, \boldsymbol{x}+\sigma \boldsymbol{h}_{i}\right)\right) & =\left(\partial_{t} v\left(t, \boldsymbol{x}, \sigma h_{i}\right)\right) \mu_{\alpha}\left(t, \boldsymbol{x}+\sigma \boldsymbol{h}_{i}\right)+v\left(t, \boldsymbol{x}, \sigma h_{i}\right) \partial_{t} \mu_{\alpha}\left(t, \boldsymbol{x}+\sigma \boldsymbol{h}_{i}\right) \\
& =T s^{|\alpha|} \theta \mathcal{O}_{\lambda, \mathfrak{K}}(1) .
\end{aligned}
$$

Next we write

$$
r(t, \boldsymbol{x}) \mathrm{A}_{i}^{2} \rho(t, \boldsymbol{x})=1+C h_{i}^{2} \int_{-1}^{1}(1-|\sigma|) r(t, \boldsymbol{x}) \partial_{i}^{2} \rho\left(t, \boldsymbol{x}+\sigma \boldsymbol{h}_{i}\right) d \sigma,
$$

which gives

$$
\partial_{t}\left(r(t, \boldsymbol{x}) \mathrm{A}_{i}^{2} \rho(t, \boldsymbol{x})\right)=C h_{i}^{2} \int_{-1}^{1}(1-|\sigma|) \partial_{t}\left(r(t, \boldsymbol{x}) \partial_{i}^{2} \rho\left(t, \boldsymbol{x}+\sigma \boldsymbol{h}_{i}\right)\right) d \sigma
$$

and the second result follows. Similarly, we write

$$
r(t, \boldsymbol{x}) \mathrm{D}_{i}^{2} \rho(t, \boldsymbol{x})=r(t, \boldsymbol{x}) \partial_{i}^{2} \rho(t, \boldsymbol{x})+C h_{i}^{4} \int_{-1}^{1}(1-|\sigma|)^{3} r(t, \boldsymbol{x}) \partial_{i}^{4} \rho\left(t, \boldsymbol{x}+\sigma \boldsymbol{h}_{i}\right) d \sigma,
$$


which gives

$$
\partial_{t}\left(r(t, \boldsymbol{x}) \mathrm{D}_{i}{ }^{2} \rho(t, \boldsymbol{x})\right)=\partial_{t}\left(r(t, \boldsymbol{x}) \partial_{i}^{2} \rho(t, \boldsymbol{x})\right)+C h_{i}^{4} \int_{-1}^{1}(1-|\sigma|)^{3} \partial_{t}\left(r(t, \boldsymbol{x}) \partial_{i}^{4} \rho\left(t, \boldsymbol{x}+\sigma \boldsymbol{h}_{i}\right)\right) d \sigma,
$$

and the third estimate follows by using Lemma 2.8 and the first result of the present proposition.

Proposition 2.15. Let $\alpha$, $\beta$ be multi-indices in the $\boldsymbol{x}$ variable, $i, j \in \llbracket 1, d \rrbracket$ and $k_{i}, k_{i}^{\prime}, k_{j}, k_{j}^{\prime} \in \mathbb{N}$. For $k_{i}+k_{j} \leqslant 2$, provided sh $\leqslant \mathfrak{K}$ we have

$$
\begin{aligned}
& \mathrm{A}_{i}{ }_{i}^{\prime} \mathrm{A}_{j}{ }^{\prime}{ }_{j}^{\prime} \mathrm{D}_{i}{ }^{k_{i}} \mathrm{D}_{j}{ }^{k_{j}} \partial^{\beta}\left(r^{2}\left(\partial^{\alpha} \rho\right){\widehat{\mathrm{D}_{i} \rho}}^{i}\right)=\partial_{i}^{k_{i}} \partial_{j}^{k_{j}} \partial^{\beta}\left(r^{2}\left(\partial^{\alpha} \rho\right) \partial_{i} \rho\right)+s^{|\alpha|+1} \mathcal{O}_{\lambda, \mathfrak{K}}\left((s h)^{2}\right) \\
& =s^{|\alpha|+1} \mathcal{O}_{\lambda, \mathfrak{K}}(1), \\
& \mathrm{A}_{i}{ }^{k_{i}^{\prime}} \mathrm{A}_{j}{ }^{k_{j}^{\prime}} \mathrm{D}_{i}{ }^{k_{i}} \mathrm{D}_{j}{ }^{k_{j}} \partial^{\beta}\left(r^{2}\left(\partial^{\alpha} \rho\right) \mathrm{A}_{i}{ }^{2} \rho\right)=\partial_{i}^{k_{i}} \partial_{j}^{k_{j}} \partial^{\beta}\left(r\left(\partial^{\alpha} \rho\right)\right)+s^{|\alpha|} \mathcal{O}_{\lambda, \mathfrak{K}}\left((s h)^{2}\right) \\
& =s^{|\alpha|} \mathcal{O}_{\lambda, \mathfrak{K}}(1), \\
& \mathrm{A}_{i}{ }^{k_{i}^{\prime}} \mathrm{A}_{j}{ }^{k_{j}^{\prime}} \mathrm{D}_{i}{ }^{k_{i}} \mathrm{D}_{j}{ }^{k_{j}} \partial^{\beta}\left(r^{2}\left(\partial^{\alpha} \rho\right) \mathrm{D}_{i}{ }^{2} \rho\right)=\partial_{i}^{k_{i}} \partial_{j}^{k_{j}} \partial^{\beta}\left(r^{2}\left(\partial^{\alpha} \rho\right) \partial_{i}^{2} \rho\right)+s^{|\alpha|+2} \mathcal{O}_{\lambda, \mathfrak{K}}\left((s h)^{2}\right) \\
& =s^{|\alpha|+2} \mathcal{O}_{\lambda, \mathfrak{K}}(1) \text {, }
\end{aligned}
$$

and we have

$$
\begin{aligned}
& \mathrm{A}_{i}{ }_{i}^{\prime} \mathrm{A}_{j}{ }^{\prime}{ }_{j}^{\prime} \mathrm{D}_{i}{ }^{k_{i}} \mathrm{D}_{j}{ }^{k_{j}} \partial^{\alpha}\left(r^{2}{\widehat{\mathrm{D}_{i} \rho}}^{i} \mathrm{D}_{j}^{2} \rho\right)=\partial_{i}^{k_{i}} \partial_{j}^{k_{j}} \partial^{\alpha}\left(r^{2}\left(\partial_{i} \rho\right) \partial_{j}^{2} \rho\right)+s^{3} \mathcal{O}_{\lambda, \mathfrak{K}}\left((s h)^{2}\right)=s^{3} \mathcal{O}_{\lambda, \mathfrak{K}}(1), \\
& \mathrm{A}_{i} k_{i}^{\prime} \mathrm{A}_{j}{ }^{\prime}{ }_{j}^{\prime} \mathrm{D}_{i}{ }^{k_{i}} \mathrm{D}_{j}{ }^{k_{j}} \partial^{\alpha}\left(r^{2}{\widehat{\mathrm{D}_{i} \rho}}^{i} \mathrm{~A}_{j}^{2} \rho\right)=\partial_{i}^{k_{i}} \partial_{j}^{k_{j}} \partial^{\alpha}\left(r \partial_{i} \rho\right)+s \mathcal{O}_{\lambda, \mathfrak{K}}\left((s h)^{2}\right)=s \mathcal{O}_{\lambda, \mathfrak{K}}(1)
\end{aligned}
$$

\section{Proof of the Carleman estimate}

Here we prove the result of Theorem 1.4. We shall carry out the proof for the operator $P^{\mathfrak{M}}=P_{-}^{\mathfrak{M}}=\partial_{t}-\mathcal{A}^{\mathfrak{M}}$. The proof is the same for $P_{+}^{\mathfrak{M}}=\partial_{t}+\mathcal{A}^{\mathfrak{M}}$ (change $t$ in to $T-t$ ). We start with some preliminary observations and notation.

Recalling the form of weight function given in (1.9) we observe that we have

$$
T^{-2} \leqslant \min _{[0, T]} \theta, \quad \frac{1}{T^{2} \delta} \underset{\delta \rightarrow 0}{\sim} \max _{[0, T]} \theta=\theta(0)=\theta(T)=\frac{1}{T^{2} \delta(1+\delta)} \leqslant \frac{1}{T^{2} \delta} .
$$

We note that

$$
\partial_{t} \theta=(2 t-T) \theta^{2} .
$$

In the proof of the Carleman estimate we shall use the following discrete gradient $\nabla=\left(D_{1}, \ldots, D_{d}\right)^{t}$ and the following notation

$$
\nabla_{\Gamma} f=\left(\sqrt{\gamma_{1}} \partial_{x_{1}} f, \ldots, \sqrt{\gamma_{d}} \partial_{x_{d}} f\right)^{t}, \quad \Delta_{\Gamma} f=\sum_{i \in \llbracket 1, d \rrbracket} \gamma_{i} \partial_{x_{i}}^{2} f .
$$

In the discrete setting we also introduce $D_{i, \Gamma} f=\sqrt{\gamma_{i}} D_{i} f, i \in \llbracket 1, d \rrbracket$, and

$$
\nabla_{\Gamma} f=\left(\sqrt{\gamma_{1}} D_{1} f, \ldots, \sqrt{\gamma_{d}} D_{d} f\right)^{t}=\left(D_{1, \Gamma} f, \ldots, D_{d, \Gamma} f\right)^{t} .
$$

The enlarged neighborhood $\tilde{\Omega}$ of $\Omega$ introduced in Assumption 1.2 allows us to apply multiple discrete operators such as $D_{i}$ and $A_{i}$ on the weight functions. In particular, this assumption yields on $\partial_{i} \Omega$

$$
\left(r{\overline{D_{i}}}^{i}\right)_{\mid k_{i}=0} \leqslant 0, \quad\left(r{\overline{D_{i} \rho}}^{i}\right)_{\mid k_{i}=N_{i}+1} \geqslant 0, \quad i \in \llbracket 1, d \rrbracket .
$$

We set $f:=P^{\mathfrak{M}} u$. At first, we shall work with the function $v=r u$, i.e., $u=\rho v$, that satisfies

$$
r\left(\partial_{t}(\rho v)+\sum_{i \in \llbracket 1, d \rrbracket} \bar{D}_{i} \gamma_{i} D_{i}(\rho v)\right)=r f .
$$


We have

$$
r \partial_{t}(\rho v)=\partial_{t} v+r\left(\partial_{t} \rho\right) v=\partial_{t} v-\tau\left(\partial_{t} \theta\right) \varphi v .
$$

Following [12], we write

$$
g=A v+B v,
$$

where $A v=A_{1} v+A_{2} v+A_{3} v, B v=B_{1} v+B_{2} v+B_{3} v$ with

$$
\begin{aligned}
g= & r f-\sum_{i \in \llbracket 1, d \rrbracket} \frac{h_{i}}{4} r{\overline{D_{i} \rho}}^{i}\left(\bar{D}_{i} \gamma_{i}\right)\left(\bar{\tau}_{i}^{+} D_{i} v-\bar{\tau}_{i}^{-} D_{i} v\right) \\
& -\sum_{i \in \llbracket 1, d \rrbracket} \frac{h_{i}^{2}}{4}\left(\bar{D}_{i} \gamma_{i}\right) r\left(\bar{D}_{i} D_{i} \rho\right){\overline{D_{i} v}}^{i}-h_{i} \sum_{i \in \llbracket 1, d \rrbracket} \mathcal{O}(1) r{\overline{D_{i} \rho}}^{i}{\overline{D_{i} v}}^{i} \\
& -\sum_{i \in \llbracket 1, d \rrbracket}\left(r\left(\bar{D}_{i} \gamma_{i}\right){\overline{D_{i} \rho}}^{i}+h_{i} \mathcal{O}(1) r\left(\bar{D}_{i} D_{i} \rho\right)\right) \overline{\tilde{v}}^{i}-2 s\left(\Delta_{\Gamma} \phi\right) v,
\end{aligned}
$$

and

$$
\begin{aligned}
& A_{1} v=\sum_{i \in \llbracket 1, d \rrbracket} r \overline{\tilde{\rho}}^{i} \bar{D}_{i}\left(\gamma_{i} D_{i} v\right), \quad A_{2} v=\sum_{i \in \llbracket 1, d \rrbracket} \gamma_{i} r\left(\bar{D}_{i} D_{i} \rho\right) \overline{\tilde{v}}^{i}, \\
& A_{3} v=-\tau\left(\partial_{t} \theta\right) \varphi v \text {, } \\
& B_{1} v=2 \sum_{i \in \llbracket 1, d \rrbracket} \gamma_{i} r{\overline{D_{i} \rho}}^{i}{\overline{D_{i} v}}^{i}, \quad B_{2} v=-2 s\left(\Delta_{\Gamma} \phi\right) v, \quad B_{3} v=\partial_{t} v .
\end{aligned}
$$

An explanation for the introduction of this additional term $B_{2} v$ is provided in [17].

Eq. (3.3) now reads $A v+B v=g$ and we write

$$
\|A v\|_{L^{2}(Q)}^{2}+\|B v\|_{L^{2}(Q)}^{2}+2(A v, B v)_{L^{2}(Q)}=\|g\|_{L^{2}(Q)}^{2}
$$

We shall need the following estimation of $\|g\|_{L^{2}(Q)}$. The proof can be adapted from Lemma 4.2 and its proof in [2] (the time dependency of the present weight function does not affect the argument and computations of the proof).

Lemma 3.1 (Estimate of the r.h.s.). For $\tau h\left(\max _{[0, T]} \theta\right) \leqslant \mathfrak{K}$ we have

$$
\|g\|_{L^{2}(Q)}^{2} \leqslant C_{\lambda, \mathfrak{K}}\left(\|r f\|_{L^{2}(Q)}^{2}+\|s v\|_{L^{2}(Q)}^{2}+h^{2}\|s \curlyvee v\|_{L^{2}(Q)}^{2}\right) .
$$

Most of the remaining of the proof will be dedicated to computing the inner-product $(A v, B v)_{L^{2}(Q)}$. Developing the inner-product $(A v, B v)_{L^{2}(Q)}$, we set $I_{i j}=\left(A_{i} v, B_{j} v\right)_{L^{2}(Q)}$.

Lemma 3.2 (Estimate of $\left.I_{11}\right)$. For $\tau h\left(\max _{[0, T]} \theta\right) \leqslant \mathfrak{K}$, the term $I_{11}$ can be estimated from below in the following way

$$
I_{11} \geqslant-\lambda^{2}\left\|(s \phi)^{\frac{1}{2}}\left|\nabla_{\Gamma} \psi\right| \nabla_{\Gamma} v\right\|_{L^{2}(\Omega)}^{2}+Y_{11}-X_{11}-W_{11}-J_{11},
$$

with

$$
Y_{11}=\sum_{i \in \llbracket 1, d \rrbracket} \iint_{Q_{i}}\left(\left(\left(\gamma_{i}^{2}+\mathcal{O}_{\lambda, \mathfrak{K}}\left((s h)^{2}\right)\right) r{\overline{D_{i} \rho}}^{i}\right)_{N_{i}+1}\left(D_{i} v\right)_{N_{i}+\frac{1}{2}}^{2}-\left(\left(\gamma_{i}^{2}+\mathcal{O}_{\lambda, \mathfrak{K}}\left((s h)^{2}\right)\right) r{\overline{D_{i} \rho}}^{i}\right)_{0}\left(D_{i} v\right)_{\frac{1}{2}}^{2}\right) d t,
$$

and

$$
X_{11}=\sum_{i \in \llbracket 1, d \rrbracket} \iint_{Q} v_{11, i}\left(D_{i} v\right)^{2} d t+\sum_{i \in \llbracket 1, d \rrbracket} \iint_{Q} \bar{v}_{11, i}\left({\overline{D_{i}}}^{i}\right)^{2} d t
$$

with $v_{11, i}$ and $\bar{v}_{11, i}$ of the form $s \lambda \phi \mathcal{O}(1)+s \mathcal{O}_{\lambda, \mathfrak{K}}(s h)$ and 


$$
W_{11}=\sum_{\substack{i, j \in \llbracket 1, d \rrbracket \\ i \neq j}} \iint_{Q} \gamma_{11, i j}\left(D_{i} D_{j} v\right)^{2} d t+\sum_{i \in \llbracket 1, d \rrbracket} \iint_{Q} \gamma_{11, i i}\left(\bar{D}_{i} D_{i} v\right)^{2} d t,
$$

with $\gamma_{11, i j}$ and $\gamma_{11, i i}$ of the form $h^{2}\left(s \lambda \phi \mathcal{O}(1)+s \mathcal{O}_{\lambda, \mathfrak{K}}(s h)\right)$ and

$$
J_{11}=\sum_{i \in \llbracket 1, d \rrbracket} \iint_{Q_{i}}\left(\left(\delta_{11, i}^{(2)}\right)_{N_{i}+\frac{1}{2}}\left(D_{i} v\right)_{N_{i}+\frac{1}{2}}^{2}+\left(\delta_{11, i}^{(2)}\right)_{\frac{1}{2}}\left(D_{i} v\right)_{\frac{1}{2}}^{2}\right) d t,
$$

with $\delta_{11, i}^{(2)}=s h_{i} \lambda \phi \mathcal{O}(1)+s h_{i} \mathcal{O}_{\lambda, \mathfrak{K}}(s h)$.

The proof follows that of Lemma 3.3 in [3]. As explained in Remark 1.3 a simplification of one of the arguments allows us not to assume convexity of $\psi$ near the boundary.

Lemma 3.3 (Estimate of $\left.I_{12}\right)$. (See Lemma 3.4 in [3].) For $\tau h\left(\max _{[0, T]} \theta\right) \leqslant \mathfrak{K}$, the term $I_{12}$ can be estimated from below in the following way

$$
I_{12} \geqslant 2 \lambda^{2}\left\|(s \phi)^{\frac{1}{2}}\left|\nabla_{\Gamma} \psi\right| \nabla_{\Gamma} v\right\|_{L^{2}(Q)}^{2}-X_{12},
$$

with

$$
X_{12}=\sum_{i \in \llbracket 1, d \rrbracket} \iint_{Q} v_{12, i}\left(D_{i} v\right)^{2} d t+\iint_{Q} \mu_{12} v^{2} d t,
$$

where $\mu_{12}=s^{2} \mathcal{O}_{\lambda, \mathfrak{K}}(1)$, and $\nu_{12, i}=s \lambda \phi \mathcal{O}(1)+s \mathcal{O}_{\lambda, \mathfrak{K}}(s h)$.

Lemma 3.4. There exists $\varepsilon_{1}(\lambda)>0$ such that, for $0<\tau h\left(\max _{[0, T]} \theta\right) \leqslant \varepsilon_{1}(\lambda)$, the term $I_{13}$ can be estimated from below in the following way

$$
I_{13} \geqslant-C_{\lambda, \mathfrak{K}} \int_{\Omega}|\nabla v(T)|^{2}-X_{13}
$$

with $C>0$ and

$$
X_{13}=\iint_{Q}\left(s(s h)+T(s h)^{2} \theta\right) \mathcal{O}_{\lambda, \mathfrak{K}}(1)|\nabla v|^{2} d t+\iint_{Q} s^{-1} \mathcal{O}_{\lambda, \mathfrak{K}}(s h)\left(\partial_{t} v\right)^{2} d t .
$$

For a proof see Appendix A.

Lemma 3.5 (Estimate of $\left.I_{21}\right)$. (See Lemma 3.5 in [3].) For $\tau h\left(\max _{[0, T]} \theta\right) \leqslant \mathfrak{K}$, the term $I_{21}$ can be estimated from below in the following way

$$
I_{21} \geqslant 3 \lambda^{4}\left\|(s \phi)^{\frac{3}{2}}\left(\nabla_{\Gamma} \psi\right)^{2} v\right\|_{L^{2}(Q)}^{2}+Y_{21}-W_{21}-X_{21},
$$

with

$$
\begin{aligned}
Y_{21} & =\sum_{i \in \llbracket 1, d \rrbracket} \int_{Q_{i}} \mathcal{O}_{\lambda, \mathfrak{K}}\left((s h)^{2}\right)\left(r{\overline{D_{i} \rho}}^{i}\right)_{0}\left(D_{i} v\right)_{\frac{1}{2}}^{2} d t+\sum_{i \in \llbracket 1, d \rrbracket} \int_{Q_{i}} \mathcal{O}_{\lambda, \mathfrak{K}}\left((s h)^{2}\right)\left(r{\overline{D_{i}} \rho}^{i}\right)_{N_{x}+1}\left(D_{i} v\right)_{N_{x}+\frac{1}{2}}^{2} d t, \\
W_{21} & =\sum_{\substack{i, j \in \llbracket 1, d \rrbracket \\
i \neq j}} \iint_{Q} \gamma_{21, i j}\left(D_{i} D_{j} v\right)^{2} d t, \quad X_{21}=\iint_{Q} \mu_{21} v^{2} d t+\sum_{i \in \llbracket 1, d \rrbracket} \iint_{Q} v_{21, i}\left(D_{i} v\right)^{2} d t,
\end{aligned}
$$

where

$$
\begin{aligned}
& \gamma_{21, i j}=h \mathcal{O}_{\lambda, \mathfrak{K}}\left((s h)^{2}\right), \quad \mu_{21}=(s \lambda \phi)^{3} \mathcal{O}(1)+s^{2} \mathcal{O}_{\lambda, \mathfrak{K}}(1)+s^{3} \mathcal{O}_{\lambda, \mathfrak{K}}(s h), \\
& v_{21, i}=s \mathcal{O}_{\lambda, \mathfrak{K}}\left((s h)^{2}\right) .
\end{aligned}
$$


Lemma 3.6 (Estimate of $\left.I_{22}\right)$. (See Lemma 3.6 in [3].) For $\tau h\left(\max _{[0, T]} \theta\right) \leqslant \mathfrak{K}$, the term $I_{22}$ is of the following form $I_{22}=-2 \lambda^{4}\left\|(s \phi)^{\frac{3}{2}}\left|\nabla_{\Gamma} \psi\right|^{2} v\right\|_{L^{2}(Q)}^{2}-X_{22}$,

with

$$
X_{22}=\iint_{Q} \mu_{22} v^{2} d t+\sum_{i \in \llbracket 1, d \rrbracket} \iint_{Q} v_{22, i}\left(D_{i} v\right)^{2} d t
$$

where $\mu_{22}=(s \lambda \phi)^{3} \mathcal{O}(1)+s^{2} \mathcal{O}_{\lambda, \mathfrak{K}}(1)+s^{3} \mathcal{O}_{\lambda, \mathfrak{K}}(s h)$, and $\nu_{22, i}=s \mathcal{O}_{\lambda, \mathfrak{K}}(s h)$.

Lemma 3.7. For $\tau h\left(\max _{[0, T]} \theta\right) \leqslant \mathfrak{K}$, the term $I_{23}$ can be estimated from below in the following way

$$
I_{23} \geqslant \int_{\Omega} s^{2}\left(\mathcal{O}_{\lambda, \mathfrak{K}}(1) v_{\mid t=T}^{2}+\mathcal{O}_{\lambda, \mathfrak{K}}(1) v_{\mid t=0}^{2}\right)-X_{23},
$$

with

$$
\begin{aligned}
X_{23}= & \iint_{Q} T s^{2} \theta \mathcal{O}_{\lambda, \mathfrak{K}}(1) v^{2} d t+\iint_{Q}(s h)^{2} s^{-1} \mathcal{O}_{\lambda, \mathfrak{K}}(1)\left(\partial_{t} v\right)^{2} d t \\
& +\sum_{i \in \llbracket 1, d \rrbracket} \iint_{Q}(s h)^{2} s \mathcal{O}_{\lambda, \mathfrak{K}}(1)\left(D_{i} v\right)^{2} d t
\end{aligned}
$$

For a proof see Appendix A.

Lemma 3.8. For $\tau h\left(\max _{[0, T]} \theta\right) \leqslant \mathfrak{K}$, the term $I_{31}$ is of the following form

$$
I_{31}=-X_{31}=\iint_{Q} T s^{2} \theta \mathcal{O}_{\lambda, \mathfrak{K}}(1) v^{2} d t+\sum_{i \in \llbracket 1, d \rrbracket} \iint_{Q} T(s h)^{2} \theta \mathcal{O}_{\lambda, \mathfrak{K}}(1)\left(D_{i} v\right)^{2} d t .
$$

For a proof see Appendix A.

With (3.1) we may write

$$
I_{32}=-X_{32}=2 \iint_{Q} \tau s\left(\partial_{t} \theta\right) \varphi\left(\Delta_{\Gamma} \phi\right) v^{2}=\iint_{Q} T s^{2} \theta \mathcal{O}_{\lambda, \mathfrak{K}}(1) v^{2} .
$$

Lemma 3.9. For $\tau h\left(\max _{[0, T]} \theta\right) \leqslant \mathfrak{K}$, the term $I_{33}$ can be estimated from below in the following way

$$
I_{33} \geqslant-X_{33}=\frac{1}{2} \tau \iint_{Q} \varphi\left(\partial_{t}^{2} \theta\right) v^{2} d t .
$$

For a proof see Appendix A.

Continuation of the proof of Theorem 1.4. Collecting the inequalities we have obtained in the previous lemmata, from (3.4) we obtain, for $0<\tau h\left(\max _{[0, T]} \theta\right) \leqslant \varepsilon_{1}(\lambda)$,

$$
\begin{aligned}
& \|A v\|_{L^{2}(Q)}^{2}+\|B v\|_{L^{2}(Q)}^{2}+2 \lambda^{4}\left\|(s \phi)^{\frac{3}{2}}\left|\nabla_{\Gamma} \psi\right|^{2} v\right\|_{L^{2}(Q)}^{2}+2 \lambda^{2}\left\|(s \phi)^{\frac{1}{2}}\left|\nabla_{\Gamma} \psi\right| \nabla_{\Gamma} v\right\|_{L^{2}(Q)}^{2}+2 Y \\
& \leqslant C_{\lambda, \mathfrak{K}}\left(\|r f\|_{L^{2}(Q)}^{2}+\int_{\Omega} s^{2}\left(v_{\mid t=T}^{2}+v_{\mid t=0}^{2}\right)+\int_{\Omega}|\nabla v|_{\mid t=T}^{2}\right)+2 X+2 W+2 J,
\end{aligned}
$$

where $C>0, Y=Y_{11}+Y_{21}$, 


$$
\begin{aligned}
X= & X_{11}+X_{12}+X_{13}+X_{21}+X_{22}+X_{23}+X_{31}+X_{32}+X_{33} \\
& +C_{\lambda, \mathfrak{K}}\left(\|s v\|_{L^{2}(Q)}^{2}+h^{2} \sum_{i \in \llbracket 1, d \rrbracket}\left\|s D_{i} v\right\|_{L^{2}(Q)}^{2}\right),
\end{aligned}
$$

$W=W_{11}+W_{21}$, and $J=J_{11}$. With the following lemma, we may in fact ignore the term $Y$. This uses the property (3.2) of the weight functions.

Lemma 3.10. (See Lemma 3.7 in [3].) For all $\lambda$ there exists $0<\varepsilon_{2}(\lambda)<\varepsilon_{1}(\lambda)$ such that for $0<\tau h\left(\max _{[0, T]} \theta\right) \leqslant$ $\varepsilon_{2}(\lambda)$, we have $Y \geqslant 0$.

Recalling that $\left|\nabla_{\Gamma} \psi\right| \geqslant C>0$ in $\Omega \backslash \omega_{0}$ we may thus write

$$
\begin{aligned}
& \|A v\|_{L^{2}(Q)}^{2}+\|B v\|_{L^{2}(Q)}^{2}+\lambda^{4}\left\|(s \phi)^{\frac{3}{2}} v\right\|_{L^{2}(Q)}^{2}+\lambda^{2}\left\|(s \phi)^{\frac{1}{2}} \nabla_{\Gamma} v\right\|_{L^{2}(Q)}^{2} \\
& \leqslant C_{\lambda, \mathfrak{K}}\left(\|r f\|_{L^{2}(Q)}^{2}+\lambda^{4}\left\|(s \phi)^{\frac{3}{2}} v\right\|_{L^{2}\left((0, T) \times \omega_{0}\right)}^{2}+\lambda^{2}\left\|(s \phi)^{\frac{1}{2}} \nabla_{\Gamma} v\right\|_{L^{2}\left((0, T) \times \omega_{0}\right)}^{2}\right. \\
& \left.\quad+\int_{\Omega} s^{2}\left(v_{\mid t=T}^{2}+v_{\mid t=0}^{2}\right)+\int_{\Omega}|\nabla v|_{\mid t=T}^{2}\right)+2 X+2 W+2 J .
\end{aligned}
$$

Lemma 3.11. We have

$$
\lambda^{2}\left\|(s \phi)^{\frac{1}{2}} \nabla_{\Gamma} v\right\|_{L^{2}(Q)}^{2} \geqslant C H-\tilde{X}-\tilde{W},
$$

for $0<h \leqslant h_{1}(\lambda)$ for some $h_{1}(\lambda)$ sufficiently small and

$$
\begin{aligned}
& H=\lambda^{2} \sum_{i \in \llbracket 1, d \rrbracket} \iint s \phi\left({\overline{D_{i}}}^{i}\right)^{2} d t+\lambda^{2} h^{2} \sum_{i \in \llbracket 1, d \rrbracket} \iint_{Q} s \phi\left(\bar{D}_{i} D_{i} v\right)^{2} d t+\lambda^{2} h^{2} \sum_{\substack{i, j \in \llbracket 1, d \rrbracket \\
i \neq j}} \iint_{Q} s \phi\left(D_{i} D_{j} v\right)^{2} d t, \\
& \tilde{X}=h^{2}\left(\sum_{i \in \llbracket 1, d \rrbracket} \iint s \mathcal{O}_{\lambda}(1)\left(D_{i} v\right)^{2} d t+\sum_{i \in \llbracket 1, d \rrbracket} \iint s \mathcal{O}_{Q}(1)\left({\overline{D_{i}}}^{i}\right)^{2} d t\right),
\end{aligned}
$$

and

$$
\tilde{W}=h^{4}\left(\sum_{\substack{i, j \in \llbracket 1, d \rrbracket \\ i \neq j}} \iint_{Q} s \mathcal{O}_{\lambda}(1)\left(D_{i} D_{j} v\right)^{2} d t+\sum_{i \in \llbracket 1, d \rrbracket} \iint_{Q} s \mathcal{O}_{\lambda}(1)\left(\bar{D}_{i} D_{i} v\right)^{2} d t\right) .
$$

For a proof see Appendix A.

If we choose $\lambda_{1} \geqslant 1$ sufficiently large, then for $\lambda=\lambda_{1}$ (fixed for the rest of the proof) and $0<\tau h\left(\max _{[0, T]} \theta\right) \leqslant$ $\varepsilon_{3}(\lambda)=\min \left(\varepsilon_{1}\left(\lambda_{1}\right), \varepsilon_{2}\left(\lambda_{1}\right)\right)$ and $0<h \leqslant h_{1}\left(\lambda_{1}\right)$, from (3.8) and Lemma 3.11, we obtain

$$
\begin{aligned}
& \|A v\|_{L^{2}(Q)}^{2}+\|B v\|_{L^{2}(Q)}^{2}+\left\|s^{\frac{3}{2}} v\right\|_{L^{2}(Q)}^{2}+\left\|s^{\frac{1}{2}} \nabla v\right\|_{L^{2}(Q)}^{2}+\underline{H} \\
& \leqslant C_{\lambda_{1}, \mathfrak{K}}\left(\|r f\|_{L^{2}(Q)}^{2}+\left\|s^{\frac{3}{2}} v\right\|_{L^{2}\left((0, T) \times \omega_{0}\right)}^{2}+\left\|s^{\frac{1}{2}} \nabla v\right\|_{L^{2}\left((0, T) \times \omega_{0}\right)}^{2}\right. \\
& \left.\quad+\int_{\Omega} s^{2}\left(v_{\mid t=T}^{2}+v_{\mid t=0}^{2}\right)+\int_{\Omega}|\nabla v|_{\mid t=T}^{2}\right)+\underline{X}+\underline{W}+\underline{J},
\end{aligned}
$$

where

$$
\underline{H}=\sum_{i \in \llbracket 1, d \rrbracket}\left\|s^{\frac{1}{2}}{\overline{D_{i}} v}^{i}\right\|_{L^{2}(Q)}^{2}+h^{2}\left(\sum_{\substack{i, j \in \llbracket 1, d \rrbracket \\ i \neq j}}\left\|s^{\frac{1}{2}} D_{i} D_{j} v\right\|_{L^{2}(Q)}^{2}+\sum_{i \in \llbracket 1, d \rrbracket}\left\|s^{\frac{1}{2}} \bar{D}_{i} D_{i} v\right\|_{L^{2}(Q)}^{2}\right),
$$




$$
\begin{aligned}
\underline{X}= & \iint_{Q} \mu_{1} v^{2} d t+\sum_{i \in \llbracket 1, d \rrbracket} \iint_{Q} v_{1, i}\left(D_{i} v\right)^{2} d t+\sum_{i \in \llbracket 1, d \rrbracket} \iint_{Q} \bar{v}_{1, i}\left({\overline{D_{i}}}^{i}\right)^{2} d t \\
& +X_{13}+X_{23}+X_{31}+X_{32}+X_{33},
\end{aligned}
$$

with $\mu_{1}=s^{2} \mathcal{O}_{\lambda_{1}, \mathfrak{K}}(1)+s^{3} \mathcal{O}_{\lambda_{1}, \mathfrak{K}}(s h)$ and $\nu_{1, i}, \bar{v}_{1, i}$, of the form $s \mathcal{O}_{\lambda_{1}, \mathfrak{K}}(s h)$, and where

$$
\underline{W}=\sum_{\substack{i, j \in \llbracket 1, d \rrbracket \\ i \neq j}} \iint_{Q} \gamma_{1, i j}\left(D_{i} D_{j} v\right)^{2} d t+\sum_{i \in \llbracket 1, d \rrbracket} \iint_{Q} \gamma_{1, i i}\left(\bar{D}_{i} D_{i} v\right)^{2} d t,
$$

where $\gamma_{1, i j}$ and $\gamma_{1, i i}$ are of the form $s h^{2} \mathcal{O}_{\lambda_{1}, \mathfrak{K}}(s h)$, and where

$$
\underline{J}=\sum_{i \in \llbracket 1, d \rrbracket} \iint_{Q_{i}}\left(\left(\delta_{1, i}\right)_{N_{i}+\frac{1}{2}}\left(D_{i} v\right)_{N_{i}+\frac{1}{2}}^{2}+\left(\delta_{1, i}\right)_{\frac{1}{2}}\left(D_{i} v\right)_{\frac{1}{2}}^{2}\right) d t,
$$

with $\delta_{1, i}=s h_{i} \mathcal{O}_{\lambda, \mathfrak{K}}(s h)$. The last term in $\underline{J}$ was obtained by "absorbing" the following term in $J_{11}$

$$
\lambda \sum_{i \in \llbracket 1, d \rrbracket} \iint_{Q_{i}} s h_{i}\left((\phi)_{N_{i}+\frac{1}{2}} \mathcal{O}(1)\left(D_{i} v\right)_{N_{i}+\frac{1}{2}}^{2}+(\phi)_{\frac{1}{2}} \mathcal{O}(1)\left(D_{i} v\right)_{\frac{1}{2}}^{2}\right) d t,
$$

by the volume term

$$
\lambda^{2} \sum_{i \in \llbracket 1, d \rrbracket} \iint_{Q} s \phi\left(D_{i} v\right)^{2} d t,
$$

for $\lambda$ large.

Observe that

$$
1 \leqslant T^{2} \theta \quad \text { and } \quad\left|\partial_{t}^{2} \theta\right| \leqslant C T^{2} \theta^{3} .
$$

We can now choose $\varepsilon_{4}$ and $h_{0}$ sufficiently small, with $0<\varepsilon_{4} \leqslant \varepsilon_{3}\left(\lambda_{1}\right), 0<h_{0} \leqslant h_{1}\left(\lambda_{1}\right)$, and $\tau_{1} \geqslant 1$ sufficiently large, such that for $\tau \geqslant \tau_{1}\left(T+T^{2}\right.$ ) (meaning in particular that $s(t)$ is taken sufficiently large), $0<h \leqslant h_{0}$, and $\tau h\left(\max _{[0, T]} \theta\right) \leqslant \varepsilon_{4}$, we obtain

$$
\begin{gathered}
\|A v\|_{L^{2}(Q)}^{2}+\|B v\|_{L^{2}(Q)}^{2}+\left\|s^{\frac{3}{2}} v\right\|_{L^{2}(Q)}^{2}+\left\|s^{\frac{1}{2}} \nabla v\right\|_{L^{2}(Q)}^{2}+\underline{H} \\
\leqslant C_{\lambda_{1}, \mathfrak{K}}\left(\|r f\|_{L^{2}(Q)}^{2}+\left\|s^{\frac{3}{2}} v\right\|_{L^{2}\left((0, T) \times \omega_{0}\right)}^{2}+\left\|s^{\frac{1}{2}} \nabla v\right\|_{L^{2}\left((0, T) \times \omega_{0}\right)}^{2}\right. \\
\left.\quad+\iint_{Q} s^{-1}(s h)\left(\partial_{t} v\right)^{2} d t+h^{-2}\left(\int_{\Omega} v_{\mid t=0}^{2}+\int v_{\Omega} v_{\mid t=T}^{2}\right)\right),
\end{gathered}
$$

where we have used that $\left(D_{i} v\right)^{2} \leqslant C h^{-2}\left(\left(\tau_{i}^{+} v\right)^{2}+\left(\tau_{i}^{-} v\right)^{2}\right)$.

Since $\tau \geqslant \tau_{1}\left(T+T^{2}\right)$ then $s(t) \geqslant \tau_{1}>0$ for any $t$, we then observe that

$$
\begin{aligned}
\left\|s^{-\frac{1}{2}} \partial_{t} v\right\|_{L^{2}(Q)}^{2} & \leqslant C_{\lambda_{1}, \mathfrak{K}}\left(\left\|s^{-\frac{1}{2}} B v\right\|_{L^{2}(Q)}^{2}+\left\|s^{\frac{1}{2}} v\right\|_{L^{2}(Q)}^{2}+\left\|s^{\frac{1}{2}} \nabla v\right\|_{L^{2}(Q)}^{2}\right) \\
& \leqslant C_{\lambda_{1}, \tau_{1}, \mathfrak{K}}\left(\|B v\|_{L^{2}(Q)}^{2}+\left\|s^{\frac{3}{2}} v\right\|_{L^{2}(Q)}^{2}+\left\|s^{\frac{1}{2}} \nabla v\right\|_{L^{2}(Q)}^{2}\right) .
\end{aligned}
$$

With $0<\varepsilon_{0} \leqslant \varepsilon_{4}$ sufficiently small and $0<\tau h\left(\max _{[0, T]} \theta\right) \leqslant \varepsilon_{0}$ we thus obtain

$$
\begin{aligned}
& \left\|s^{-\frac{1}{2}} \partial_{t} v\right\|_{L^{2}(Q)}^{2}+\left\|s^{\frac{3}{2}} v\right\|_{L^{2}(Q)}^{2}+\left\|s^{\frac{1}{2}} \nabla v\right\|_{L^{2}(Q)}^{2}+\underline{H} \\
& \quad \leqslant C_{\lambda_{1}, \tau_{1}, \mathfrak{K}}\left(\|r f\|_{L^{2}(Q)}^{2}+\left\|s^{\frac{3}{2}} v\right\|_{L^{2}\left((0, T) \times \omega_{0}\right)}^{2}+\left\|s^{\frac{1}{2}} \nabla v\right\|_{L^{2}\left((0, T) \times \omega_{0}\right)}^{2}+h^{-2}\left(\int_{\Omega} v_{\mid t=0}^{2}+\int_{\Omega} v_{\mid t=T}^{2}\right)\right) .
\end{aligned}
$$


Arguing as at the end of the proof of Theorem 4.1 in [2] (using Lemma 4.9 therein) for the spatial discrete derivative and as in [12] for the time derivative, we obtain

$$
\begin{aligned}
& \tau^{3}\left\|\theta^{\frac{3}{2}} e^{\tau \theta \varphi} u\right\|_{L^{2}(Q)}^{2}+\tau \sum_{i \in \llbracket 1, d \rrbracket}\left\|\theta^{\frac{1}{2}} e^{\tau \theta \varphi} D_{i} u\right\|_{L^{2}(Q)}^{2}+\tau^{-1}\left\|\theta^{-\frac{1}{2}} e^{\tau \theta \varphi} \partial_{t} u\right\|_{L^{2}(Q)}^{2}+\underline{H} \\
& \leqslant C_{\lambda_{1}, \tau_{1}, \mathfrak{K}}\left(\left\|e^{\tau \theta \varphi} P^{\mathfrak{M}} u\right\|_{L^{2}(Q)}^{2}+\tau^{3}\left\|\theta^{\frac{3}{2}} e^{\tau \theta \varphi} u\right\|_{L^{2}\left((0, T) \times \omega_{1}\right)}^{2}+\tau \sum_{i \in \llbracket 1, d \rrbracket}\left\|\theta^{\frac{1}{2}} e^{\tau \theta \varphi} D_{i} u\right\|_{L^{2}\left((0, T) \times \omega_{1}\right)}^{2}\right. \\
& \left.\quad+h^{-2}\left(\left|e^{\tau \theta \varphi} u_{\mid t=0}\right|_{L^{2}(\Omega)}^{2}+\left|e^{\tau \theta \varphi} u_{\mid t=T}\right|_{L^{2}(\Omega)}^{2}\right)\right),
\end{aligned}
$$

with $\omega_{0} \Subset \omega_{1} \Subset \omega$.

We next remove the volume norms $\tau\left\|\theta^{\frac{1}{2}} e^{\tau \theta \varphi} D_{i} u\right\|_{L^{2}\left((0, T) \times \omega_{1}\right)}^{2}$ in the r.h.s. through the following estimate.

Lemma 3.12. We have

$$
\begin{gathered}
\tau \sum_{i \in \llbracket 1, d \rrbracket}\left\|\theta^{\frac{1}{2}} e^{\tau \theta \varphi} D_{i} u\right\|_{L^{2}\left((0, T) \times \omega_{1}\right)}^{2} \\
\leqslant C\left(\left\|e^{\tau \theta \varphi} f\right\|_{L^{2}(Q)}^{2}+\tau^{3}\left\|\theta^{\frac{3}{2}} e^{\tau \theta \varphi} u\right\|_{L^{2}((0, T) \times \omega)}^{2}+\sum_{i \in \llbracket 1, d \rrbracket}\left\|e^{\tau \theta \varphi} D_{i} u\right\|_{L^{2}(Q)}^{2}\right. \\
\left.+h^{-1}\left|e^{\tau \theta \varphi} u_{\mid t=0}\right|_{L^{2}(\Omega)}^{2}+h^{-1}\left|e^{\tau \theta \varphi} u_{\mid t=T}\right|_{L^{2}(\Omega)}^{2}\right) .
\end{gathered}
$$

For a proof see Appendix A.

For $\tau_{2} \geqslant \tau_{1}$ sufficiently large, for $\tau \geqslant \tau_{2}\left(T+T^{2}\right)$, we thus obtain

$$
\begin{aligned}
& \tau^{3}\left\|\theta^{\frac{3}{2}} e^{\tau \theta \varphi} u\right\|_{L^{2}(Q)}^{2}+\tau \sum_{i \in \llbracket 1, d \rrbracket}\left\|\theta^{\frac{1}{2}} e^{\tau \theta \varphi} D_{i} u\right\|_{L^{2}(Q)}^{2}+\tau^{-1}\left\|\theta^{-\frac{1}{2}} e^{\tau \theta \varphi} \partial_{t} u\right\|_{L^{2}(Q)}^{2}+\underline{H} \\
& \leqslant C_{\lambda_{1}, \tau_{1}, \mathfrak{K}}\left(\left\|e^{\tau \theta \varphi} P^{\mathfrak{M}} u\right\|_{L^{2}(Q)}^{2}+\tau^{3}\left\|\theta^{\frac{3}{2}} e^{\tau \theta \varphi} u\right\|_{L^{2}((0, T) \times \omega)}^{2}\right. \\
&\left.\quad+h^{-2}\left(\left|e^{\tau \theta \varphi} u_{\mid t=0}\right|_{L^{2}(\Omega)}^{2}+\left|e^{\tau \theta \varphi} u_{\mid t=T}\right|_{L^{2}(\Omega)}^{2}\right)\right) .
\end{aligned}
$$

With Lemmata 2.2 and 2.3 and we now write

$$
\begin{aligned}
{\overline{D_{i} u}}^{i} & ={\overline{D_{i}(\rho v)}}^{i}={\overline{\left(D_{i} \rho\right) \tilde{v}^{i}}}^{i}+\overline{\tilde{\rho}}^{i} D_{i} v \\
& ={\overline{D_{i} \rho}}^{i}{\overline{v^{i}}}^{i}+\overline{\tilde{\rho}}^{i}{\overline{D_{i} v}}^{i}+\frac{h_{i}^{2}}{4}\left(\left(\bar{D}_{i} D_{i} \rho\right){\overline{D_{i} v}}^{i}+{\overline{D_{i} \rho}}^{i} \bar{D}_{i} D_{i} v\right) \\
& ={\overline{D_{i} \rho}}^{i} v+\overline{\tilde{\rho}}^{i}{\overline{D_{i} v}}^{i}+\frac{h_{i}^{2}}{4}\left(\left(\bar{D}_{i} D_{i} \rho\right){\overline{D_{i} v}}^{i}+2{\overline{D_{i} \rho}}^{i} \bar{D}_{i} D_{i} v\right)
\end{aligned}
$$

With Proposition 2.10 we then find

$$
r{\overline{D_{i} u}}^{i}=s v \mathcal{O}_{\lambda, \mathfrak{K}}(1)+{\overline{D_{i}}}^{i} \mathcal{O}_{\lambda, \mathfrak{K}}(1)+s h_{i}^{2}\left(\bar{D}_{i} D_{i} v\right) \mathcal{O}_{\lambda, \mathfrak{K}}(1) .
$$

With (3.12) and (3.13) and the expression (3.10) of $\underline{H}$ we then obtain

$$
\begin{aligned}
& \tau^{3}\left\|\theta^{\frac{3}{2}} e^{\tau \theta \varphi} u\right\|_{L^{2}(Q)}^{2}+\tau \sum_{i \in \llbracket 1, d \rrbracket}\left(\left\|\theta^{\frac{1}{2}} e^{\tau \theta \varphi} D_{i} u\right\|_{L^{2}(Q)}^{2}+\left\|\theta^{\frac{1}{2}} e^{\tau \theta \varphi}{\overline{D_{i} u}}^{i}\right\|_{L^{2}(Q)}^{2}\right)+\tau^{-1}\left\|\theta^{-\frac{1}{2}} e^{\tau \theta \varphi} \partial_{t} u\right\|_{L^{2}(Q)}^{2} \\
& \quad \leqslant C_{\lambda_{1}, \tau_{1}, \mathfrak{K}}\left(\left\|e^{\tau \theta \varphi} P^{\mathfrak{M}} u\right\|_{L^{2}(Q)}^{2}+\tau^{3}\left\|\theta^{\frac{3}{2}} e^{\tau \theta \varphi} u\right\|_{L^{2}((0, T) \times())}^{2}+h^{-2}\left(\left|e^{\tau \theta \varphi} u_{\mid t=0}\right|_{L^{2}(\Omega)}^{2}+\left|e^{\tau \theta \varphi} u_{\mid t=T}\right|_{L^{2}(\Omega)}^{2}\right)\right) .
\end{aligned}
$$

Finally, we observe that since $\max _{[0, T]} \theta \leqslant \frac{1}{\delta T^{2}(1+\delta)} \leqslant \frac{1}{T^{2} \delta}$, a sufficient condition for $\tau h\left(\max _{[0, T]} \theta\right) \leqslant \varepsilon_{0}$ becomes $\tau h\left(T^{2} \delta\right)^{-1} \leqslant \varepsilon_{0}$. This concludes the proof of Theorem 1.4. 


\section{4. $h$-null controllability: the linear case}

We consider the following semi-discrete parabolic problem with potential

$$
\partial_{t} y+\mathcal{A}^{\mathfrak{M}} y+a y=\mathbf{1}_{\omega} v, \quad t \in(0, T), \quad y_{\mid \partial \Omega}=0 .
$$

To achieve a $h$-null controllability result for (4.1) we start by proving a relaxed observability estimate.

\subsection{A relaxed observability estimate}

The adjoint system associated with the controlled system with potential (4.1) is given by

$$
-\partial_{t} q+\mathcal{A}^{\mathfrak{M}} q+a q=0, \quad t \in(0, T), \quad q \mid \partial \Omega=0 .
$$

With the Carleman estimate we proved in Theorem 1.4 we have the following relaxed observability estimate.

Proposition 4.1. There exists positive constants $C_{0}, C_{1}$ and $C_{2}$ such that for all $T>0$ and all potential function a, under the condition $h \leqslant \min \left(h_{0}, h_{1}\right)$ with

$$
h_{1}=C_{0}\left(1+\frac{1}{T}+\|a\|_{\infty}^{\frac{2}{3}}\right)^{-1}
$$

any solution of (1.12) satisfies

$$
|q(0)|_{L^{2}(\Omega)}^{2} \leqslant C_{\mathrm{obs}}^{2}\left(\|q\|_{L^{2}((0, T) \times \omega)}^{2}+e^{-\frac{C_{1}}{h}}|q(T)|_{L^{2}(\Omega)}^{2}\right),
$$

with $C_{\mathrm{obs}}=e^{C_{2}\left(1+\frac{1}{T}+T\|a\|_{\infty}+\|a\|_{\infty}^{\frac{2}{3}}\right)}$.

Remark 4.2. The power $2 / 3$ in $C_{\text {obs }}$ above is essential in the limitation $r<3 / 2$ for the semi-linearities we consider. See Section 1.2.3; see also the sections below. The question of the optimality of this power is then natural. In [8] it was proven to be optimal in the case of a system of parabolic equations. For a single parabolic equation this question remains open.

Proof. The change of variable

$$
\tilde{q}=e^{\|a\|_{\infty}(t-T)} q,
$$

allows us to consider the potential $a$ to be non-negative.

With the Carleman estimate we proved in Theorem 1.4 we have

$$
\left\|s^{\frac{3}{2}} e^{s \varphi} q\right\|_{L^{2}(Q)}^{2} \leqslant C\left(\left\|e^{s \varphi} a q\right\|_{L^{2}(Q)}^{2}+\left\|s^{\frac{3}{2}} e^{s \varphi} q\right\|_{L^{2}((0, T) \times \omega)}^{2}+h^{-2}\left(\left|e^{s \varphi} q_{\mid t=0}\right|_{L^{2}(\Omega)}^{2}+\left|e^{s \varphi} q_{\mid t=T}\right|_{L^{2}(\Omega)}^{2}\right)\right),
$$

with $s=\tau \theta$ for $\tau \geqslant \tau_{0}\left(T+T^{2}\right), 0<h \leqslant h_{0}$ and $\tau h\left(\delta T^{2}\right)^{-1} \leqslant \varepsilon_{0}$.

As $1 \leqslant C \theta T^{2}$ it suffices to have

$$
\tau \geqslant C T^{2}\|a\|_{\infty}^{\frac{2}{3}}
$$

to obtain

$$
\left\|s^{\frac{3}{2}} e^{s \varphi} q\right\|_{L^{2}(Q)}^{2} \leqslant C\left(\left\|s^{\frac{3}{2}} e^{s \varphi} q\right\|_{L^{2}((0, T) \times \omega)}^{2}+h^{-2}\left(\left|e^{s \varphi} q_{\mid t=0}\right|_{L^{2}(\Omega)}^{2}+\left|e^{s \varphi} q_{\mid t=T}\right|_{L^{2}(\Omega)}^{2}\right)\right) .
$$

We thus choose $\tau_{1} \geqslant \tau_{0}$ sufficiently large to have (4.5) for

$$
\tau \geqslant \tau_{1}\left(T+T^{2}+T^{2}\|a\|_{\infty}^{\frac{2}{3}}\right) .
$$

The positivity of $\mathcal{A}^{\mathfrak{M}}+a$ yields

$$
|q(0)|_{L^{2}} \leqslant|q(t)|_{L^{2}}, \quad t \in(0, T) .
$$


Recalling that $\varphi$ is negative, and independent of time $t$, we observe that we have

$$
\begin{aligned}
\int_{0}^{T} \int_{\Omega} s^{3} e^{2 s \varphi} q(t)^{2} d t & \geqslant \int_{\frac{T}{4}}^{\frac{3 T}{4}} \int_{\Omega} s^{3} e^{2 s \varphi} q(t)^{2} d t \\
& \geqslant \int_{\frac{T}{4}}^{\frac{3 T}{4}} \tau^{3} \theta\left(\frac{T}{2}\right)^{3} e^{2 \tau \theta\left(\frac{T}{4}\right) \inf \varphi}|q(0)|_{L^{2}(\Omega)}^{2} d t \\
& =\frac{1}{2} T \tau^{3} \theta\left(\frac{T}{2}\right)^{3} e^{2 \tau \theta\left(\frac{T}{4}\right) \inf \varphi}|q(0)|_{L^{2}(\Omega)}^{2} \\
& \geqslant C T e^{-C^{\prime} \frac{\tau}{T^{2}}|q(0)|_{L^{2}(\Omega)}^{2},}
\end{aligned}
$$

as $\tau \geqslant \tau_{0} T^{2}$.

As $\theta(T)=\theta(0)=\left(T^{2}(1+\delta) \delta\right)^{-1}$, we have $e^{s \varphi_{\mid t=0}}=e^{s \varphi_{\mid t=T}} \leqslant e^{C \frac{\tau}{\delta T^{2}}(\sup \varphi)}$ and we find

$$
\begin{aligned}
\left|e^{s \varphi} q_{\mid t=0}\right|_{L^{2}(\Omega)}^{2}+\left|e^{s \varphi} q_{\mid t=T}\right|_{L^{2}(\Omega)}^{2} & \leqslant C e^{-\frac{C \tau}{\delta T^{2}}}\left(|q(0)|_{L^{2}(\Omega)}^{2}+|q(T)|_{L^{2}(\Omega)}^{2}\right) \\
& \leqslant C^{\prime} e^{-\frac{C \tau}{\delta T^{2}}}|q(T)|_{L^{2}(\Omega)}^{2},
\end{aligned}
$$

as $\sup \varphi<0$, and using (4.8). We now write

$$
\left\|s^{\frac{3}{2}} e^{s \varphi} q\right\|_{L^{2}((0, T) \times \omega)}^{2} \leqslant C e^{\tau \theta\left(\frac{T}{2}\right) \sup \varphi}\|q\|_{L^{2}((0, T) \times \omega)}^{2} .
$$

Consequently we obtain

$$
T|q(0)|_{L^{2}(\Omega)}^{2} \leqslant C e^{C \frac{\tau}{T^{2}}}\|q\|_{L^{2}((0, T) \times \omega)}^{2}+h^{-2} e^{\frac{\tau}{T^{2}}\left(C-\frac{C^{\prime}}{\delta}\right)}|q(T)|_{L^{2}(\Omega)}^{2} .
$$

For $0<\delta \leqslant \delta_{1} \leqslant \delta_{0}$, with $\delta_{1}$ sufficiently small, we obtain

$$
T|q(0)|_{L^{2}(\Omega)}^{2} \leqslant C e^{C \frac{\tau}{T^{2}}}\|q\|_{L^{2}((0, T) \times \omega)}^{2}+h^{-2} e^{-C^{\prime} \frac{\tau}{\delta T^{2}}}|q(T)|_{L^{2}(\Omega)}^{2} .
$$

We recall the conditions of Theorem 1.4:

$$
\frac{\tau h}{\delta T^{2}} \leqslant \varepsilon_{0} \quad \text { and } \quad h \leqslant h_{0} .
$$

They need to be fulfilled along with $\delta \leqslant \delta_{1}$.

We fix $\tau=\tau_{0}\left(T+T^{2}+T^{2}\|a\|_{\infty}^{\frac{2}{3}}\right)$ with $\tau_{0}$ as chosen in Theorem 1.4. We define $h_{1}$ through

$$
h_{1}=\frac{\varepsilon_{0}}{\tau_{0}} \delta_{1}\left(1+\frac{1}{T}+\|a\|_{\infty}^{\frac{2}{3}}\right)^{-1},
$$

which gives

$$
\frac{\tau h_{1}}{\delta_{1} T^{2}}=\varepsilon_{0} .
$$

We choose $h \leqslant \min \left(h_{0}, h_{1}\right)$, and $\delta=h \delta_{1} / h_{1} \leqslant \delta_{1}$ we then find $\frac{\tau h}{\delta T^{2}}=\varepsilon_{0}$.

As $\tau /\left(T^{2} \delta\right)=\varepsilon_{0} / h$, we obtain from (4.10)

$$
|q(0)|_{L^{2}(\Omega)}^{2} \leqslant C e^{C\left(1+\frac{1}{T}+\|a\|_{\infty}^{\frac{2}{3}}\right)}\|q\|_{L^{2}((0, T) \times \omega)}^{2}+h^{-2} e^{-\frac{C^{\prime} \varepsilon_{0}}{h}}|q(T)|_{L^{2}(\Omega)}^{2},
$$

which gives

$$
|q(0)|_{L^{2}(\Omega)}^{2} \leqslant e^{C^{\prime \prime}\left(1+\frac{1}{T}+\|a\|_{\infty}^{\frac{2}{3}}\right)}\|q\|_{L^{2}((0, T) \times \omega)}^{2}+e^{-\frac{C^{\prime \prime \prime}}{h}}|q(T)|_{L^{2}(\Omega)}^{2} .
$$

Recalling that we made the change of variable (4.4) we conclude the proof. 


\section{2. h-null controllability}

With the result of Proposition 4.1 we deduce the following $h$-null controllability result for system (4.1).

Proposition 4.3. There exist positive constants $C_{1}, C_{2}, C_{3}$ and for $T>0$ a map $L_{T, a}: \mathbb{R}^{\mathfrak{M}} \rightarrow L^{2}\left(0, T ; \mathbb{R}^{\mathfrak{M}}\right)$, such that if $h \leqslant \min \left(h_{0}, h_{1}\right)$, with $h_{1}$ as given in Proposition 4.1 , for all initial data $y_{0} \in \mathbb{R}^{\mathfrak{M}}$, there exists a semi-discrete control function $v$ given by $v=L_{T, a}\left(y_{0}\right)$ such that the solution to (4.1) satisfies

$$
|y(T)|_{L^{2}(\Omega)} \leqslant C_{\mathrm{obs}} e^{-C_{2} / h}\left|y_{0}\right|_{L^{2}(\Omega)}, \quad \text { and } \quad\|v\|_{L^{2}(Q)} \leqslant C_{\mathrm{obs}}\left|y_{0}\right|_{L^{2}(\Omega)},
$$

with $C_{\mathrm{obs}}$ as given in Proposition 4.1.

Remark 4.4. Note that the final state is of size $e^{-C / h}\left|y_{0}\right|_{L^{2}(\Omega)}$. In comparison the result obtained in [2,3] based on a Lebeau-Robbiano-type spectral inequality yields a final state of size $e^{-C / h^{2}}\left|y_{0}\right|_{L^{2}(\Omega)}$. The method in [2,3] does not yield however a precise observability constant as in Proposition 4.1 which is crucial in the study of semi-linear equation as we do below. Questions regarding differences in size of the final state when comparing this two method are of theoretical interest: can one improve the estimate given above? Yet for practical purposes there are very little differences: in fact one is rather interested in a target of size $h^{p}\left|y_{0}\right|_{L^{2}(\Omega)}$ in connexion with the consistency of the numerical scheme. We refer to $[4,6]$ where such questions appear.

Proof. We use a dual formulation; we consider the adjoint parabolic equation

$$
\left(-\partial_{t}+\mathcal{A}^{\mathfrak{M}}\right) q+a q=0, \quad q(T)=q_{T} .
$$

The relaxed observability inequality of Proposition 4.1 gives

$$
|q(0)|_{L^{2}(\Omega)}^{2} \leqslant C_{\mathrm{obs}}^{2}\left(\|q\|_{L^{2}((0, T) \times \omega)}^{2}+\varepsilon\left|q_{T}\right|_{L^{2}(\Omega)}^{2}\right),
$$

with $C_{\mathrm{obs}}=e^{C\left(1+\frac{1}{T}+T\|a\|_{\infty}+\|a\|_{\infty}^{\frac{2}{3}}\right)}$ and $\varepsilon=e^{-\frac{C^{\prime}}{h}}$. We introduce the functional

$$
J\left(q_{T}\right)=\frac{1}{2} \int_{0}^{T}|q(t)|_{L^{2}(\omega)}^{2} d t+\frac{\varepsilon}{2}\left|q_{T}\right|_{L^{2}(\Omega)}^{2}+\left\langle y_{0}, q(0)\right\rangle .
$$

The functional $J$ is smooth, strictly convex, and coercive on a finite dimensional space, thus it admits a unique minimizer $q_{T}=q_{T}^{o p t}$. We denote by $q^{o p t}(t)$ the associated solution of the adjoint problem (4.11). The Euler-Lagrange equation associated with this minimization problem reads

$$
\int_{0}^{T}\left\langle q^{o p t}(t), q(t)\right\rangle_{L^{2}(\omega)} d t+\varepsilon\left\langle q_{T}^{o p t}, q_{T}\right\rangle_{L^{2}(\Omega)}=-\left\langle y_{0}, q(0)\right\rangle_{L^{2}(\Omega)},
$$

for any $q_{T} \in \mathbb{R}^{\mathfrak{M}}$, with the associated solution $q(t)$ of the adjoint problem (4.11). We set the control to $v=L_{T, a}\left(y_{0}\right)=$ $\mathbf{1}_{\omega} q^{\text {opt }}(t)$. We consider now the solution $y$ to the controlled problem

$$
\partial_{t} y+\mathcal{A}^{\mathfrak{M}} y+a y=\mathbf{1}_{\omega} q^{o p t}(t), \quad t \in(0, T), \quad y_{\mid \partial \Omega}=0, \quad y(0)=y_{0} .
$$

By multiplying this equation by $q$ and integrating by parts, we deduce

$$
\int_{0}^{T}\left\langle q^{o p t}(t), q(t)\right\rangle_{L^{2}(\omega)} d t=\left\langle y(T), q_{T}\right\rangle_{L^{2}(\Omega)}-\left\langle y_{0}, q(0)\right\rangle_{L^{2}(\Omega)},
$$

for any $q_{T} \in \mathbb{R}^{\mathfrak{M}}$. With (4.14) we conclude that

$$
y(T)=-\varepsilon q_{T}^{o p t}
$$


We now take $q_{T}=q_{T}^{\text {opt }}$ in (4.14) to obtain

$$
\left\|q^{o p t}\right\|_{L^{2}((0, T) \times \omega)}^{2}+\varepsilon\left|q_{T}^{o p t}\right|_{L^{2}(\Omega)}^{2}=-\left\langle y_{0},\left.q^{o p t}(0)\right|_{L^{2}(\Omega)} \leqslant\left|y_{0}\right|_{L^{2}(\Omega)}\left|q^{o p t}(0)\right|_{L^{2}(\Omega)} .\right.
$$

With the observability inequality (4.12) we have

$$
\left|q^{o p t}(0)\right|_{L^{2}(\Omega)}^{2} \leqslant C_{\mathrm{obs}}^{2}\left(\left\|q^{o p t}\right\|_{L^{2}((0, T) \times \omega)}^{2}+\varepsilon\left|q_{T}^{o p t}\right|_{L^{2}(\Omega)}^{2}\right),
$$

leading to

$$
\varepsilon^{\frac{1}{2}}\left|q_{T}^{o p t}\right|_{L^{2}(\Omega)} \leqslant C_{\text {obs }}\left|y_{0}\right|_{L^{2}(\Omega)},
$$

and

$$
\|v\|_{L^{2}((0, T) \times \omega)}=\left\|q^{o p t}\right\|_{L^{2}((0, T) \times \omega)} \leqslant C_{\mathrm{obs}}\left|y_{0}\right|_{L^{2}(\Omega)} .
$$

Hence the linear map

$$
\begin{aligned}
L_{T, a}: L^{2}(\Omega) & \rightarrow L^{2}((0, T) \times \omega), \\
y_{0} & \mapsto v,
\end{aligned}
$$

is well defined and continuous.

With (4.15) and (4.16) we obtain

$$
|y(T)|_{L^{2}(\Omega)} \leqslant C_{\text {obs }} e^{-C / h}\left|y_{0}\right|_{L^{2}(\Omega)},
$$

which concludes the proof.

\section{5. $h$-null controllability: the semi-linear case}

We start this section by stating a classical regularity result for the linear equation

$$
\partial_{t} y+\mathcal{A}^{\mathfrak{M}} y+a y=f \in L^{2}\left(0, T ; \mathbb{R}^{\mathfrak{M}}\right), \quad y_{\mid \partial \Omega}=0, \quad y(0)=y_{0} \in \mathbb{R}^{\mathfrak{M}} .
$$

Proposition 5.1. For any $a \in L^{\infty}$ the solution to (5.1) satisfies

$$
\begin{aligned}
& \|y\|_{L^{\infty}\left(0, T, L^{2}(\Omega)\right)}+\|y\|_{L^{2}(Q)}+\sum_{i \in \llbracket 1, d \rrbracket}\left\|D_{i} y\right\|_{L^{2}(Q)} \leqslant K_{0}\left(\left|y_{0}\right|_{L^{2}(\Omega)}+\|f\|_{L^{2}(Q)}\right), \\
& \left\|\partial_{t} y\right\|_{L^{2}(Q)}+\sum_{i \in \llbracket 1, d \rrbracket}\left\|D_{i} y\right\|_{L^{\infty}\left(0, T, L^{2}(\Omega)\right)} \leqslant K_{1}\left(\sum_{i \in \llbracket 1, d \rrbracket}\left|D_{i} y_{0}\right|_{L^{2}(\Omega)}+\|f\|_{L^{2}(Q)}\right),
\end{aligned}
$$

with $K_{0}=e^{C\left(1+T+T\|a\|_{L} \infty\right)}$ and $K_{1}=e^{C\left(1+T+\left(T^{\frac{1}{2}}+T\right)\|a\|_{L} \infty\right)}$.

We now consider the semi-linear equation

$$
\left(\partial_{t}+\mathcal{A}^{\mathfrak{M}}\right) y+\mathcal{G}(y)=f, \quad t \in(0, T), \quad y_{\mid \partial \Omega}=0, \quad y(0)=y_{0},
$$

with $\mathcal{G}$ Lipschitz continuous. Since $\mathbb{R}^{\mathfrak{M}}$ is finite dimensional, the Cauchy-Lipschitz theorem applies. For each initial data $y_{0}$ and r.h.s. $f L^{1}$ w.r.t. $t$, this yields the existence and uniqueness of maximal solution in $\mathscr{C}^{1}\left(\left[0, t_{0}\right) ; \mathbb{R}^{\mathfrak{M}}\right)$ with $0<t_{0} \leqslant T$. If $t_{0}<T$ the solution ceases to exist at $t=t_{0}$ because of a blow-up: $\lim _{t \rightarrow t_{0}^{-}}|y(t)|_{L^{\infty}}=+\infty$.

We shall consider the following semi-linear semi-discrete control problem

$$
\left(\partial_{t}+\mathcal{A}^{\mathfrak{M}}\right) y+\mathcal{G}(y)=\mathbf{1}_{\omega} v, \quad t \in(0, T), \quad y_{\mid \partial \Omega}=0, \quad y(0)=y_{0},
$$

where $\omega \subset \Omega$. The function $\mathcal{G}: \mathbb{R} \rightarrow \mathbb{R}$ is assumed ${ }^{1}$ of the form

$$
\mathcal{G}(y)=y g(y), \quad y \in \mathbb{R},
$$

\footnotetext{
1 Regularity as low as locally Lipschitz can be considered. For results with lower regularity in the continuous case we refer to [11].
} 
with $g$ Lipschitz continuous. In Section 5.1 we shall assume that $g \in L^{\infty}(\mathbb{R})$, i.e., the semi-linearity is sub-linear. In Section 5.2, following [11], we shall consider the more general case of a possibly super-linear semi-linearity (see (5.9)).

\subsection{The sub-linear case}

In the present section we assume that $g \in L^{\infty}(\mathbb{R})$. The sublinearity of the function $\mathcal{G}$ prevents any blow-up as can be observed by the Gronwall inequality. Solutions to (5.2) are thus defined on $[0, T]$.

We prove the following $h$-null controllability result.

Theorem 5.2. There exist positive constants $C_{0}, C_{1}$ such that for all $T>0$ and $h$ chosen sufficiently small, for all initial data $y_{0} \in \mathbb{R}^{\mathfrak{M}}$, there exists a semi-discrete control function $v$ with

$$
\|v\|_{L^{2}(Q)} \leqslant C\left|y_{0}\right|_{L^{2}(\Omega)},
$$

such that the solution to the semi-linear parabolic equation (5.3) satisfies

$$
|y(T)|_{L^{2}(\Omega)} \leqslant C e^{-C_{0} / h}\left|y_{0}\right|_{L^{2}(\Omega)},
$$

with $C=e^{C_{1}\left(1+\frac{1}{T}+T\|g\|_{\infty}+\|g\|_{\infty}^{\frac{2}{3}}\right)}$.

Observe that the constants are uniform with respect to the discretization parameter $h$. In particular the $L^{2}$-norm of the control function $v$ remains bounded as $h$ varies. Then, up to a subsequence, the semi-discrete controls weakly converge towards a function $v \in L^{2}((0, T) \times \omega)$. Moreover, since we consider here the sub-linear case, the corresponding subsequence of solutions $y$ can be shown to be compact in $L^{2}((0, T) \times \Omega)$ for instance (this follows from a suitable discrete $L^{2}\left(0, T, H^{1}(\Omega)\right)$-estimate and from a time-translation estimate). Therefore, it is possible to show that, at the limit, $v$ is a null-control for the parabolic problem under study. However, since such a null-control is certainly not unique, we are not able to prove convergence of the whole sequence of semi-discrete controls. Additionally, even for linear problems, it is not known in general whether this convergence is strong or not (see for instance the discussion in $[19,6])$.

Proof. The proof follows that given by [16] with some particularities due to the discrete case. We set $Z=$ $L^{2}\left(0, T ; \mathbb{R}^{\mathfrak{M}}\right)$. For $z \in Z$ we consider the linear control problem

$$
\left(\partial_{t}+\mathcal{A}^{\mathfrak{M}}\right) y+y g(z)=\mathbf{1}_{\omega} v, \quad y_{\mid \partial \Omega}=0, \quad y(0)=y_{0} .
$$

We set $a_{z}=g(z)$. We have $\left\|a_{z}\right\|_{L^{\infty}(Q)} \leqslant\|g\|_{\infty}$. If we apply Proposition 4.3, with $h$ chosen sufficiently small, i.e., $h \leqslant h_{0}$ and

$$
h \leqslant C_{0}\left(1+\frac{1}{T}+\|g\|_{\infty}^{\frac{2}{3}}\right)^{-1}
$$

we denote by $v_{z}=L_{T, a_{z}}\left(y_{0}\right) \in L^{2}\left(0, T ; \mathbb{R}^{\mathfrak{M}}\right)$ and $y_{z}$ the associated control function and controlled solution. We have

$$
\left|y_{z}(T)\right|_{L^{2}(\Omega)} \leqslant C e^{-C_{0} / h}\left|y_{0}\right|_{L^{2}(\Omega)}, \quad\left\|v_{z}\right\|_{L^{2}(Q)} \leqslant C\left|y_{0}\right|_{L^{2}(\Omega)},
$$

for $C_{0}>0$ and $C=e^{C_{1}\left(1+\frac{1}{T}+T\|g\|_{\infty}+\|g\|_{\infty}^{\frac{2}{3}}\right)}$, uniform with respect to $z$ and the discretization parameter $h$.

With the regularity result of Proposition 5.1 we can define the map

$$
\begin{aligned}
\Lambda: Z & \rightarrow Z, \\
z & \mapsto y_{z},
\end{aligned}
$$

and, as $T$ is fixed and $\left\|a_{z}\right\|_{L^{\infty}(Q)} \leqslant\|g\|_{L^{\infty}}$ we have

$$
\|\Lambda z\|_{L^{2}(Q)}=\left\|y_{z}\right\|_{L^{2}(Q)} \leqslant C\left(\left|y_{0}\right|_{L^{2}(\Omega)}+\left\|v_{z}\right\|_{L^{2}(Q)}\right) \leqslant C^{\prime}\left|y_{0}\right|_{L^{2}(\Omega)},
$$

where the constants only depend on $T$ and $\|g\|_{L^{\infty}}$ (the sublinearity property of $\mathcal{G}$ is used here). 
Hence, $\Lambda$ maps the closed ball $B$ of $Z$ of radius $R=C^{\prime}\left|y_{0}\right|_{L^{2}(\Omega)}$ into itself.

Lemma 5.3. The map $\Lambda$ is continuous on $Z$.

The proof of Lemma 5.3 is given below.

Recalling the form of the difference operator $D$ we find

$$
\left|D y_{0}\right|_{L^{2}(\Omega)} \leqslant C h^{-1}\left|y_{0}\right|_{L^{2}(\Omega)} .
$$

Additionally from Proposition 5.1 we find that

$$
\begin{aligned}
& \left\|y_{z}\right\|_{L^{2}(Q)} \leqslant C\left(\left|y_{0}\right|_{L^{2}(\Omega)}+\left\|v_{z}\right\|_{L^{2}(Q)}\right) \leqslant C^{\prime}\left|y_{0}\right|_{L^{2}(\Omega)}, \\
& \left\|\partial_{t} y_{z}\right\|_{L^{2}(Q)} \leqslant C\left(\left|D y_{0}\right|_{L^{2}(\Omega)}+\left\|v_{z}\right\|_{L^{2}(Q)}\right) \leqslant C\left(h^{-1}+1\right)\left|y_{0}\right|_{L^{2}(\Omega)} .
\end{aligned}
$$

As $H^{1}(0, T)$ injects compactly in $L^{2}(0, T)$ and $\mathbb{R}^{\mathfrak{M}}$ is finite dimensional we obtain that $\Lambda(B)$ is relatively compact in $Z$.

All the previous properties allow us to apply the Schauder topological fixed-point theorem: there exists $y \in Z$ such that $\Lambda(y)=y$. Setting $v=L_{T, a_{y}}\left(y_{0}\right)$ we obtain

$$
\left(\partial_{t}+\mathcal{A}^{\mathfrak{M}}\right) y+y g(y)=\mathbf{1}_{\omega} v, \quad y_{\mid \partial \Omega}=0, \quad y(0)=y_{0},
$$

which concludes the proof as we have found a control $v$ that drives the solution of the semi-linear semi-discrete parabolic system to a final state $y(T)$ with the estimates of (5.6).

Proof of Lemma 5.3. With the continuity and the boundedness of $g$ we have that the map $z \mapsto a_{z}=g(z)$ is continuous on $Z$ with values in the space $\tilde{Z}=\left\{a \in Z,\|a\|_{\infty} \leqslant\|g\|_{\infty}\right\}$.

Let us consider the following controlled parabolic problems

$$
\left\{\begin{array} { l } 
{ ( \partial _ { t } + \mathcal { A } ^ { \mathfrak { M } } ) y _ { 1 } + a _ { 1 } y _ { 1 } = \mathbf { 1 } _ { \omega } v _ { a _ { 1 } } , } \\
{ y _ { 1 | t = 0 } = y _ { 0 } , }
\end{array} \quad \left\{\begin{array}{l}
\left(\partial_{t}+\mathcal{A}^{\mathfrak{M}}\right) y_{2}+a_{2} y_{2}=\mathbf{1}_{\omega} v_{a_{2}}, \\
y_{2 \mid t=0}=y_{0},
\end{array}\right.\right.
$$

with $a_{1}, a_{2} \in \tilde{Z}$. The controls $v_{a_{2}}$ and $v_{a_{1}}$ are obtained through Proposition 4.3. Setting $Y=y_{2}-y_{1}$ we write

$$
\left(\partial_{t}+\mathcal{A}^{\mathfrak{M}}\right) Y+a_{1} Y=\mathbf{1}_{\omega}\left(v_{a_{2}}-v_{a_{1}}\right)+\left(a_{1}-a_{2}\right) y_{2}, \quad Y_{\mid t=0}=0
$$

From Proposition 5.1 we obtain

$$
\|Y\|_{L^{\infty}\left(0, T, L^{2}(\Omega)\right)} \leqslant C\left(\left\|v_{a_{2}}-v_{a_{1}}\right\|_{L^{2}(Q)}+\left\|y_{2}\right\|_{L^{\infty}(Q)}\left\|a_{2}-a_{1}\right\|_{L^{2}(Q)}\right) .
$$

As we have

$$
\left\|y_{2}\right\|_{L^{\infty}(Q)} \leqslant C_{h}\left\|y_{2}\right\|_{L^{\infty}\left(0, T, L^{2}(\Omega)\right)} \leqslant C_{h}^{\prime}\left(\left|y_{0}\right|_{L^{2}(\Omega)}+\left\|v_{a_{2}}\right\|_{L^{2}(Q)}\right)
$$

and $\left\|v_{a_{2}}\right\|_{L^{2}(Q)} \leqslant C\left|y_{0}\right|_{L^{2}(\Omega)}$ we obtain

$$
\left\|y_{2}-y_{1}\right\|_{L^{\infty}\left(0, T, L^{2}(\Omega)\right)} \leqslant C_{h}\left(\left\|v_{a_{2}}-v_{a_{1}}\right\|_{L^{2}(Q)}+\left|y_{0}\right|_{L^{2}(\Omega)}\left\|a_{2}-a_{1}\right\|_{L^{2}(Q)}\right) .
$$

To prove the result of the lemma it thus suffices to prove that the map $a \mapsto v_{a}$ is continuous on $\tilde{Z}$.

As in the proof of 4.3 we consider the adjoint parabolic equation

$$
\left(-\partial_{t}+\mathcal{A}^{\mathfrak{M}}\right) q+a q=0, \quad q(T)=q_{T},
$$

and we denote by $Q\left(a, q_{T}\right)$ its solution. The control $v_{a}$ of the parabolic system

$$
\left(\partial_{t}+\mathcal{A}^{\mathfrak{M}}\right) y+a y=\mathbf{1}_{\omega} v_{a}, \quad y_{\mid t=0}=y_{0},
$$

is then given by $v_{a}=\mathbf{1}_{\omega} Q\left(a, q_{T}^{\text {opt,a}}\right)$, with $q_{T}^{\text {opt }, a}$ minimizer of the functional (4.13). We shall thus study the continuity of the map $a \in \tilde{Z} \mapsto \mathbf{1}_{\omega} Q\left(a, q_{T}^{o p t, a}\right) \in Z$. 
For the two potentials $a_{2}$ and $a_{1}$ we can write the associated Euler-Lagrange equations for the two associated minimizers

$$
\begin{aligned}
& \int_{0}^{T}\left\langle Q\left(a_{1}, q_{T}^{o p t, a_{1}}\right), Q\left(a_{1}, \tilde{q}_{T}\right)\right\rangle_{L^{2}(\omega)} d t+\varepsilon\left\langle q_{T}^{o p t, a_{1}}, \tilde{q}_{T}\right\rangle_{L^{2}(\Omega)}+\left\langle Q\left(a_{1}, \tilde{q}_{T}\right)(0), y_{0}\right\rangle_{L^{2}(\Omega)}=0, \\
& \int_{0}^{T}\left\langle Q\left(a_{2}, q_{T}^{o p t, a_{2}}\right), Q\left(a_{2}, \tilde{q}_{T}\right)\right\rangle_{L^{2}(\omega)} d t+\varepsilon\left\langle q_{T}^{o p t, a_{2}}, \tilde{q}_{T}\right\rangle_{L^{2}(\Omega)}+\left\langle Q\left(a_{2}, \tilde{q}_{T}\right)(0), y_{0}\right\rangle_{L^{2}(\Omega)}=0,
\end{aligned}
$$

for any $\tilde{q}_{T} \in L^{2}(\Omega)$. Choosing $\tilde{q}_{T}=q_{T}^{\text {opt }, a_{1}}-q_{T}^{\text {opt, } a_{2}}$ and subtracting these two equations yields

$$
\begin{aligned}
&\left\|Q\left(a_{1}, q_{T}^{o p t, a_{1}}\right)-Q\left(a_{2}, q_{T}^{o p t, a_{2}}\right)\right\|_{L^{2}\left(0, T, L^{2}(\omega)\right)}^{2}+\varepsilon\left\|q_{T}^{o p t, a_{1}}-q_{T}^{o p t, a_{2}}\right\|_{L^{2}(\Omega)}^{2} \\
&=-\left\langle Q\left(a_{1}, q_{T}^{o p t, a_{1}}-q_{T}^{o p t, a_{2}}\right)(0)-Q\left(a_{2}, q_{T}^{o p t, a_{1}}-q_{T}^{o p t, a_{2}}\right)(0), y_{0}\right\rangle_{L^{2}(\Omega)} \\
&+\int_{0}^{T}\left\langle Q\left(a_{1}, q_{T}^{o p t, a_{1}}\right), Q\left(a_{1}, q_{T}^{o p t, a_{2}}\right)-Q\left(a_{2}, q_{T}^{o p t, a_{2}}\right)\right\rangle_{L^{2}(\omega)} d t \\
&-\int_{0}^{T}\left\langle Q\left(a_{2}, q_{T}^{o p t, a_{2}}\right), Q\left(a_{1}, q_{T}^{o p t, a_{1}}\right)-Q\left(a_{2}, q_{T}^{o p t, a_{1}}\right)\right\rangle_{L^{2}(\omega)} d t .
\end{aligned}
$$

Applying Proposition 5.1 to the adjoint system (5.8) with $q_{T}=q_{T}^{\text {opt, } a}$ and using that $a \in \tilde{Z}$, we have

$$
\left\|Q\left(a, q_{T}^{o p t, a}\right)\right\|_{L^{\infty}(Q)} \leqslant C\left\|Q\left(a, q_{T}^{o p t, a}\right)\right\|_{L^{\infty}\left(0, T, L^{2}(\Omega)\right)} \leqslant C^{\prime}\left|q_{T}^{o p t, a}\right|_{L^{2}(\Omega)} \leqslant C^{\prime \prime} \varepsilon^{-\frac{1}{2}}\left|y_{0}\right|_{L^{2}(\Omega)},
$$

by (4.16). We thus find

$$
\begin{aligned}
& \left\|Q\left(a_{1}, q_{T}^{o p t, a_{1}}\right)-Q\left(a_{2}, q_{T}^{o p t, a_{2}}\right)\right\|_{L^{2}\left(0, T, L^{2}(\omega)\right)}^{2}+\varepsilon\left|q_{T}^{o p t, a_{1}}-q_{T}^{o p t, a_{2}}\right|_{L^{2}(\Omega)}^{2} \\
& \leqslant C_{\varepsilon, T}\left|y_{0}\right|_{L^{2}(\Omega)}\left(\left|Q\left(a_{1}, q_{T}^{o p t, a_{1}}-q_{T}^{o p t, a_{2}}\right)(0)-Q\left(a_{2}, q_{T}^{o p t, a_{1}}-q_{T}^{o p t, a_{2}}\right)(0)\right|_{L^{2}(\Omega)}\right. \\
& \quad+\left\|Q\left(a_{1}, q_{T}^{o p t, a_{2}}\right)-Q\left(a_{2}, q_{T}^{o p t, a_{2}}\right)\right\|_{L^{2}(Q)} \\
& \left.\quad+\left\|Q\left(a_{1}, q_{T}^{o p t, a_{1}}\right)-Q\left(a_{2}, q_{T}^{o p t, a_{1}}\right)\right\|_{L^{2}(Q)}\right) .
\end{aligned}
$$

Using now (5.7) for the adjoint system and again (4.16) we obtain

$$
\begin{aligned}
& \left\|Q\left(a_{1}, q_{T}^{o p t, a_{1}}\right)-Q\left(a_{2}, q_{T}^{o p t, a_{2}}\right)\right\|_{L^{2}\left(0, T, L^{2}(\omega)\right)}^{2}+\varepsilon\left|q_{T}^{o p t, a_{1}}-q_{T}^{o p t, a_{2}}\right|_{L^{2}(\Omega)}^{2} \\
& \quad \leqslant C_{\varepsilon, T}^{\prime}\left|y_{0}\right|_{L^{2}(\Omega)}^{2}\left\|a_{1}-a_{2}\right\|_{L^{2}} .
\end{aligned}
$$

This gives the continuity of the map $a \mapsto \mathbf{1}_{\omega} Q\left(a, q_{T}^{\text {opt,a}}\right)$ on $\tilde{Z}$ and thus of the map $a \mapsto v_{a}$ on $\tilde{Z}$. This concludes the proof.

\subsection{The super-linear case}

In this section we consider also the semi-linear semi-discrete control problem (5.3). The function $\mathcal{G}$ is still assumed of the form (5.4) but, in agreement with the controllability result of [11] in the continuous case, $g$ is now assumed to satisfy

$$
|g(y)| \leqslant K \ln ^{r}(e+|y|), \quad y \in \mathbb{R}, \text { with } 0 \leqslant r<\frac{3}{2} .
$$

To ease the notation we set

$$
\zeta(s)=K \ln ^{r}(e+s) \text { for } s \geqslant 0 .
$$




\subsubsection{Preliminary observations}

If $T>0$, for a vanishing r.h.s. $f$, starting from a sufficiently small initial data ensures the existence of the solution of (5.2) in the time interval $[0, T]$. Moreover the size of the solution at time $t=T$ remains small.

Proposition 5.4. Let $T>0$. There exists $M_{0}>0$ and $K_{0}>0$ such that the maximal solution to

$$
\left(\partial_{t}+\mathcal{A}^{\mathfrak{M}}\right) y+\mathcal{G}(y)=0, \quad y_{\mid \partial \Omega}=0, \quad y(0)=y_{0}
$$

satisfies

$$
|y(t)|_{L^{2}(\Omega)} \leqslant\left|y_{0}\right|_{L^{2}(\Omega)} e^{K_{0} t}, \quad 0<t<T,
$$

if we choose $h^{-d / 2}\left|y_{0}\right|_{L^{2}(\Omega)} \leqslant M_{0}$.

This result will be useful for the construction of the control function in the proofs below: if a sufficiently small state is achieved for a time $0<t_{1}<T$ it suffices to set the control function to 0 for time interval $\left(t_{1}, T\right)$ and one still obtains a small solution at the final time $T$.

Proof. The maximal solution to (5.11) can cease to exist if there is a blow-up at some time $t_{0} \in(0, T]$. We first prove that this does not occur if either $r \leqslant 1$ or if the initial condition is chosen sufficiently small.

Taking the $L^{2}$-inner product of the equation with $y(t)$ we have, after a discrete integration by parts,

$$
\frac{1}{2} \partial_{t}|y(t)|_{L^{2}(\Omega)}^{2}+\sum_{i \in \llbracket 1, d \rrbracket}\left\langle\gamma_{i} D_{i} y(t),\left.D_{i} y(t)\right|_{L^{2}(\Omega)}+\left\langle\mathcal{G}(y(t)),\left.y(t)\right|_{L^{2}(\Omega)}=0,\right.\right.
$$

for $0 \leqslant t<t_{0}$, which gives with (5.9)-(5.10),

$$
\begin{aligned}
\frac{1}{2} \partial_{t}|y(t)|_{L^{2}(\Omega)}^{2} & \leqslant|g(y(t))|_{L^{\infty}(\Omega)}|y(t)|_{L^{2}(\Omega)}^{2} \\
& \leqslant \zeta\left(|y(t)|_{L^{\infty}(\Omega)}\right)|y(t)|_{L^{2}(\Omega)}^{2} \\
& \leqslant \zeta\left(c_{0} h^{-d / 2}|y(t)|_{L^{2}(\Omega)}\right)|y(t)|_{L^{2}(\Omega)}^{2}
\end{aligned}
$$

using that if $u \in \mathbb{R}^{\mathfrak{M}},|u|_{L^{\infty}} \leqslant c_{0} h^{-d / 2}|u|_{L^{2}}$, for some $c_{0}>0$. Setting $z(t)=h^{-d}|y(t)|_{L^{2}(\Omega)}^{2}$ we obtain

$$
z^{\prime} \leqslant 2 z \zeta\left(c_{0} z^{\frac{1}{2}}\right)
$$

We have $z(t) \geqslant 0$ and if $z\left(t_{1}\right)=0$ for some $t_{1}$ then $z$ vanishes identically. We may thus assume that $z>0$ on $\left[0, t_{0}\right)$.

We set $\rho(s)=\left(2 s \zeta\left(c_{0} s^{\frac{1}{2}}\right)\right)^{-1}$ for $s \in(0,+\infty)$ and $\mu(s)=\int_{1}^{s} \rho(\sigma) d \sigma$. Recall that $\zeta$ is defined in (5.10). We have $\frac{d}{d t} \mu(z(t)) \leqslant 1$, which gives

$$
\mu(z(t))-\mu(z(0)) \leqslant t, \quad \forall 0 \leqslant t<t_{0} .
$$

Notice that $\rho$ is not integrable at $0^{+}$and that $\mu(1)=0$. Therefore, there exists a unique $M_{0}>0$ such that $\mu\left(M_{0}^{2}\right)=-T$.

We now consider two cases:

Case $r \leqslant 1$. We have $\lim _{z \rightarrow+\infty} \mu(s)=+\infty$. Assuming that $\lim _{t \rightarrow t_{0}^{-}} z(t)=+\infty$, with inequality (5.12) we reach a contradiction. Hence the solution does not blow up in finite time.

Case $r>1$. In this case the function $\rho$ is integrable at infinity. Assuming that $\lim _{t \rightarrow t_{0}^{-}} z(t)=+\infty$, with (5.12) we find

$$
\lim _{s \rightarrow \infty} \mu(s)-\mu(z(0)) \leqslant t_{0} .
$$

If $z(0) \leqslant M_{0}^{2}$ then $\mu(z(0)) \leqslant \mu\left(M_{0}^{2}\right)=-T \leqslant-t_{0}$ and therefore we obtain $\lim _{s \rightarrow \infty} \mu(s) \leqslant 0$. This prevents a possible blow-up at time $t_{0}$ and thus the solution exists for $t \in[0, T]$. 
In both cases, if $z(0)^{\frac{1}{2}}=h^{-d / 2}\left|y_{0}\right|_{L^{2}} \leqslant M_{0}$, then the solution exists on $[0, T]$, and moreover we have $\mu(z(t)) \leqslant$ $T+\mu(z(0)) \leqslant 0$ which implies that $z(t) \leqslant 1$ for any $t \in[0, T]$, uniformly w.r.t. $h$.

There exists $K_{0}>0$ such that $s^{-1} \leqslant 2 K_{0} \rho(s)$, for any $s \in(0,1]$. This yields by integration

$$
\ln \left(\frac{z(t)}{z(0)}\right) \leqslant 2 K_{0}(\mu(z(t))-\mu(z(0))) \leqslant 2 K_{0} t
$$

Hence we have $z(t) \leqslant z(0) e^{2 K_{0} t}$ which gives the result.

\subsubsection{Controllability result}

We shall prove the following theorem.

Theorem 5.5. Let $\mathcal{G}$ satisfy (5.4) and (5.9). There exists $C_{0}>0$ such that for $T>0$ and $M>0$ there exist positive constants $C, h_{2}>0$ and $\alpha_{0}$, such that for $0<h \leqslant h_{2}$ and all initial data $y_{0} \in \mathbb{R}^{\mathfrak{M}}$, with $\left|y_{0}\right|_{L^{2}(\Omega)} \leqslant M$, there exists a semi-discrete control function $v$ such that the solution to the semi-linear parabolic equation (5.3) satisfies

$$
|y(T)|_{L^{2}(\Omega)} \leqslant C e^{-C_{0} / h}\left|y_{0}\right|_{L^{2}(\Omega)}, \quad \text { and } \quad\|v\|_{L^{2}(Q)} \leqslant C_{h}\left|y_{0}\right|_{L^{2}(\Omega)},
$$

where $C_{h}=C h^{-\alpha_{0}}$ with $C=C(T, M)$.

Remark 5.6. Note that the constant $C_{0}$ that yields the exponential decay of the final state when the discretization is refined is independant of $T$ and $M$, i.e., the size of the initial condition.

Observe that the constant $C_{h}$ in the estimation of the control norm is not uniform with respect to $h$. Here we cannot bound the norm of the control if the discretization is refined, i.e., if $h$ decreases to 0 . To achieve a proper estimate one should make use of a control $v$ in $L^{\infty}\left(0, T ; \mathbb{R}^{\mathfrak{M}}\right)$. This approach was central in the proof of the the controllability of semi-linear parabolic equations in [11]. To that purpose one needs to refine the observability inequality of Proposition 4.1. This is the subject of future work based on the analysis of the semi-discrete heat kernel. Such an estimation will also naturally yield a local controllability result. In dimension $d>1$ with such an estimation we can replace $h^{-d / 2}$ by a constant in (5.23).

Yet, only using an $L^{2}$ control, the result of Theorem 5.5 can be improved if we consider the case of one dimension in space. This is presented in Theorem 5.11 in Section 5.2.3 below. In fact in this case the heat kernel estimation can be replaced by a (discrete) Sobolev inequality.

Remark 5.7. Note that the largest discretization step $h$ allowed by the previous theorem is function of the norm of the initial condition of the control problem.

Proof of Theorem 5.5. We use some of the arguments given by [11], yet with some particularities due to the discrete case.

Let $R_{0}>0$ be such that $\zeta\left(R_{0}\right) \geqslant 1$. For $R>R_{0}$ we introduce

$$
S_{R}(s)= \begin{cases}s & \text { if }-R \leqslant s \leqslant R \\ \operatorname{sgn}(s) R & \text { otherwise. }\end{cases}
$$

Adapting [11] we introduce the following control time

$$
T_{R}=\min \left(T, \zeta(R)^{-2 / 3}\right) .
$$

We set $Z_{R}=L^{\infty}\left(0, T_{R} ; \mathbb{R}^{\mathfrak{M}}\right)$ and $Q_{R}=\left(0, T_{R}\right) \times \mathbb{R}^{\mathfrak{M}}$. We shall denote by $\|\cdot\|_{L^{p}\left(Q_{R}\right)}$ the natural norm on $L^{p}\left(0, T_{R} ; \mathbb{R}^{\mathfrak{M}}\right), p=2$ or $p=\infty$ (see the end of Section 1.1.4).

For $z \in Z_{R}$ we set $a_{z}=g\left(S_{R}(z)\right)$. Observe that we have

$$
\frac{1}{T_{R}} \leqslant \frac{1}{T}+\zeta(R)^{2 / 3}, \quad\left(T_{R}^{\frac{1}{2}}+T_{R}\right)\left\|a_{z}\right\|_{\infty} \leqslant 2 \zeta(R)^{2 / 3},
$$


since

$$
\left|a_{z}(t, \boldsymbol{k})\right|=\left|g\left(S_{R}(z(t, \boldsymbol{k}))\right)\right| \leqslant \zeta\left(S_{R}(z(t, \boldsymbol{k}))\right) \leqslant \zeta(R) \quad \forall t, \forall \boldsymbol{k},
$$

and $\zeta(R) \geqslant \zeta\left(R_{0}\right) \geqslant 1$. We shall choose $R$ in the form $R=R(h)=C h^{-\alpha} \geqslant R_{0}$, with $\alpha$ and $C$ to be made precise below (Lemma 5.9).

For $z \in Z_{R}$ we consider the linear control problem on $\left[0, T_{R}\right]$ :

$$
\left(\partial_{t}+\mathcal{A}^{\mathfrak{M}}\right) y+y a_{z}=\mathbf{1}_{\omega} v, \quad y_{\mid \partial \Omega}=0, \quad y(0)=y_{0} .
$$

If we apply Proposition 4.3 to the control system (5.14), we set

$$
v_{R, z}=L_{T_{R}, a_{z}}\left(y_{0}\right) \quad 0<t \leqslant T_{R},
$$

as the associated control function and we denote by $y_{R, z}$ the controlled solution.

We have

$$
\begin{aligned}
& \left|y_{R, z}\left(T_{R}\right)\right|_{L^{2}(\Omega)} \leqslant K_{2} e^{-\tilde{C}_{0} / h}\left|y_{0}\right|_{L^{2}(\Omega)}, \\
& \left\|v_{R, z}\right\|_{L^{2}\left(0, T_{R}, L^{2}(\Omega)\right)} \leqslant K_{2}\left|y_{0}\right|_{L^{2}(\Omega)},
\end{aligned}
$$

with

$$
K_{2}=e^{C\left(1+\frac{1}{T_{R}}+T_{R}\left\|a_{z}\right\|_{\infty}+\left\|a_{z}\right\|_{\infty}^{\frac{2}{3}}\right)} \leqslant e^{C^{\prime}\left(1+\frac{1}{T}+\zeta(R)^{2 / 3}\right)},
$$

by (5.13), for $C>0$ and $C^{\prime}>0$, uniform with respect to $z$ and the discretization parameter $h$.

To apply Proposition 4.3 we require $h \leqslant C\left(1+\frac{1}{T_{R}}+\left\|a_{z}\right\|_{\infty}^{2 / 3}\right)^{-1}$. As $\left\|a_{z}\right\|_{\infty} \leqslant \zeta(R)$ and using (5.13) we see that it suffices to have

$$
h \leqslant C^{\prime}\left(1+\frac{1}{T}+\zeta(R)^{2 / 3}\right)^{-1} .
$$

As here $R=R(h)$, checking that (5.18) holds will be a key step. This is done in Lemma 5.9 below.

As $y_{R, z} \in L^{\infty}\left(0, T_{R} ; \mathbb{R}^{\mathfrak{M}}\right)$ by Proposition 5.1 (using that $\mathbb{R}^{\mathfrak{M}}$ is finite dimensional) the following map is well defined

$$
\begin{aligned}
\Lambda_{R}: Z_{R} & \rightarrow Z_{R}, \\
z & \mapsto y_{R, z} .
\end{aligned}
$$

Lemma 5.8. The map $\Lambda_{R}$ is continuous on $Z_{R}=L^{\infty}\left(0, T_{R} ; \mathbb{R}^{\mathfrak{M}}\right)$.

The proof of Lemma 5.8 is given below.

We denote by $B_{R, h}$ the ball centered at 0 and of radius $R=R(h)$ in $Z_{R}$. Proposition 5.1 and (5.16) give

$$
\left\|y_{R, z}\right\|_{L^{\infty}\left(Q_{R}\right)} \leqslant C_{h}\left\|y_{R, z}\right\|_{L^{\infty}\left(0, T ; L^{2}(\Omega)\right)} \leqslant C_{h}^{\prime}\left(\left|y_{0}\right|_{L^{2}(\Omega)}+\left\|v_{R, z}\right\|_{L^{2}(Q)}\right) \leqslant C_{h}^{\prime \prime}\left|y_{0}\right|_{L^{2}(\Omega)},
$$

and

$$
\left\|\partial_{t} y_{R, z}\right\|_{L^{2}\left(0, T_{R} ; L^{\infty}(\Omega)\right)} \leqslant C_{h}\left\|\partial_{t} y_{R, z}\right\|_{L^{2}\left(Q_{R}\right)} \leqslant C_{h}^{\prime}\left(\left|D y_{0}\right|_{L^{2}(\Omega)}+\left\|v_{R, z}\right\|_{L^{2}(Q)}\right) \leqslant C_{h}^{\prime \prime}\left|y_{0}\right|_{L^{2}(\Omega)} .
$$

As $H^{1}\left(0, T_{R}\right)$ injects compactly in $L^{\infty}\left(0, T_{R}\right)$ and $\mathbb{R}^{\mathfrak{M}}$ is finite dimensional we find that $\Lambda\left(B_{R, h}\right)$ is relatively compact in $L^{\infty}\left(0, T_{R} ; \mathbb{R}^{\mathfrak{M}}\right)$.

Lemma 5.9. Let $\alpha>d / 2$. For any $M>0$, there exists $C=C(M, \alpha)>0$ and $\tilde{h}_{2}=\tilde{h}_{2}(T, M, \alpha)$, such that for

$$
R=C h^{-\alpha},
$$

condition (5.18) is fulfilled and the map $\Lambda_{R}$ maps $B_{R, h}$ into itself if $0<h \leqslant \tilde{h}_{2}$ and if $\left|y_{0}\right|_{L^{2}(\Omega)} \leqslant M$. 
The proof of Lemma 5.9 is given below.

All the previous properties allow us to apply the Schauder topological fixed-point theorem if $0<h \leqslant \tilde{h}_{2}$ and $\left|y_{0}\right|_{L^{2}(\Omega)} \leqslant M$ and $R$ is chosen according to Lemma 5.9: there exists $y \in B_{R, h}$ such that $\Lambda_{R}(y)=y$. Setting $v_{R}=$ $L_{T_{R}, a_{y}}\left(y_{0}\right)$ we obtain

$$
\left(\partial_{t}+\mathcal{A}^{\mathfrak{M}}\right) y+y a_{y}=\mathbf{1}_{\omega} v_{R}, \quad 0<t \leqslant T_{R}, \quad y_{\mid \partial \Omega}=0, \quad y(0)=y_{0} .
$$

Since $y \in B_{R, h}$ we have $\|y\|_{L^{\infty}(Q)} \leqslant R$. Then we have $a_{y}=g\left(S_{R}(y)\right)=g(y)$, which yields

$$
\left(\partial_{t}+\mathcal{A}^{\mathfrak{M}}\right) y+\mathcal{G}(y)=\mathbf{1}_{\omega} v_{R}, \quad 0<t \leqslant T_{R}, \quad y_{\mid \partial \Omega}=0, \quad y(0)=y_{0} .
$$

With the value of $R=R(h)$ given by Lemma 5.9 we go back to the estimations (5.15)-(5.16) and find

$$
\left|y\left(T_{R}\right)\right|_{L^{2}(\Omega)} \leqslant K_{2} e^{-\tilde{C}_{0} / h}\left|y_{0}\right|_{L^{2}(\Omega)}, \quad\left\|v_{R}\right\|_{L^{2}\left(Q_{R}\right)} \leqslant K_{2}\left|y_{0}\right|_{L^{2}(\Omega)},
$$

with (use (5.24) in the proof of Lemma 5.9 and that $2 r / 3 \leqslant 1$ )

$$
K_{2} \leqslant e^{C\left(1+\frac{1}{T}+\zeta(R)^{2 / 3}\right)} \leqslant e^{2 C \zeta(R)^{2 / 3}} \leqslant e^{C^{\prime} \ln (e+R)^{2 r / 3}} \leqslant(e+R)^{C^{\prime \prime}} \leqslant C^{\prime \prime}(M) h^{-\alpha_{0}},
$$

with $\alpha_{0}>0$. This yields

$$
\left|y\left(T_{R}\right)\right|_{L^{2}(\Omega)} \leqslant C(M) e^{-C_{0} / h}\left|y_{0}\right|_{L^{2}(\Omega)}, \quad\left\|v_{R}\right\|_{L^{2}\left(Q_{R}\right)} \leqslant C(M) h^{-\alpha_{0}}\left|y_{0}\right|_{L^{2}(\Omega)},
$$

for any $0<C_{0}<\tilde{C}_{0}$.

We now define $v$ on $[0, T]$ by

$$
v= \begin{cases}v_{R} & \text { if } 0<t \leqslant T_{R}, \\ 0 & \text { if } T_{R}<t \leqslant T,\end{cases}
$$

We naturally have $\|v\|_{L^{2}(Q)} \leqslant C h^{-\alpha_{0}}\left|y_{0}\right|_{L^{2}(\Omega)}$.

If we have

$$
h^{-d / 2}\left|y\left(T_{R}\right)\right|_{L^{2}(\Omega)} \leqslant M_{0},
$$

we can apply Proposition 5.4 on the time interval ${ }^{2}\left[T_{R}, T\right]$, which yields

$$
|y(T)|_{L^{2}(\Omega)} \leqslant\left|y\left(T_{R}\right)\right|_{L^{2}(\Omega)} e^{K_{0}\left(T-T_{R}\right)} \leqslant C(M, T) e^{-C_{0} / h}\left|y_{0}\right|_{L^{2}(\Omega)} .
$$

With (5.20), choosing $h_{2} \leqslant \min \left(h_{0}, \tilde{h}_{2}\right)$ sufficiently small, condition (5.21) can be fulfilled if $0<h \leqslant h_{2}$, which concludes the proof.

Proof of Lemma 5.8. (Continuity of the map $\Lambda_{R}$ on $L^{\infty}\left(0, T_{R} ; \mathbb{R}^{\mathfrak{M}}\right)$.) In this proof the values of $h$ and $R$ are kept fixed.

Observe that $z \mapsto S_{R}(z)$ is continuous on $Z_{R}$ as $S_{R}$ is Lipschitz continuous. As $g$ is also Lipschitz continuous we have that the map $z \mapsto a_{z}$ is continuous on $Z_{R}$ as well.

Let us consider the following controlled parabolic problems

$$
\left\{\begin{array} { l } 
{ ( \partial _ { t } + \mathcal { A } ^ { \mathfrak { M } } ) y _ { 1 } + a _ { 1 } y _ { 1 } = \mathbf { 1 } _ { \omega } v _ { a _ { 1 } } , } \\
{ y _ { 1 | t = 0 } = y _ { 0 } , }
\end{array} \quad \left\{\begin{array}{l}
\left(\partial_{t}+\mathcal{A}^{\mathfrak{M}}\right) y_{2}+a_{2} y_{2}=\mathbf{1}_{\omega} v_{a_{2}}, \\
y_{2 \mid t=0}=y_{0},
\end{array}\right.\right.
$$

with $\max \left(\left\|a_{1}\right\|_{\infty},\left\|a_{2}\right\|_{\infty}\right) \leqslant \zeta(R)$. The controls $v_{a_{2}}$ and $v_{a_{1}}$ are obtained by Proposition 4.3. Setting $Y=y_{2}-y_{1}$ we write

$$
\left(\partial_{t}+\mathcal{A}^{\mathfrak{M}}\right) Y+a_{1} Y=\mathbf{1}_{\omega}\left(v_{a_{2}}-v_{a_{1}}\right)+\left(a_{1}-a_{2}\right) y_{2}, \quad Y_{\mid t=0}=0
$$

\footnotetext{
${ }^{2}$ Here $R=R(h)$. Yet, Proposition 5.4 applies in fact on the interval $\left[T_{R}, T_{R}+T\right]$ by translation in time.
} 
From Proposition 5.1 we obtain

$$
\begin{aligned}
\|Y\|_{L^{\infty}\left(Q_{R}\right)} & \leqslant C_{h}\|Y\|_{L^{\infty}\left(0, T_{R}, L^{2}(\Omega)\right)} \\
& \leqslant C_{h}^{\prime}\left(\left\|v_{a_{2}}-v_{a_{1}}\right\|_{L^{2}\left(Q_{R}\right)}+\left\|y_{2}\right\|_{L^{2}\left(Q_{R}\right)}\left\|a_{2}-a_{1}\right\|_{L^{\infty}\left(Q_{R}\right)}\right) .
\end{aligned}
$$

As we have

$$
\left\|y_{2}\right\|_{L^{2}\left(Q_{R}\right)} \leqslant C\left(\left|y_{0}\right|_{L^{2}(\Omega)}+\left\|v_{a_{2}}\right\|_{L^{2}\left(Q_{R}\right)}\right)
$$

and $\left\|v_{a_{2}}\right\|_{L^{2}\left(Q_{R}\right)} \leqslant C_{R}\left|y_{0}\right|_{L^{2}(\Omega)}$ we obtain

$$
\left\|y_{2}-y_{1}\right\|_{L^{\infty}\left(Q_{R}\right)} \leqslant C_{h, R}\left(\left\|v_{a_{2}}-v_{a_{1}}\right\|_{L^{2}\left(Q_{R}\right)}+\left|y_{0}\right|_{L^{2}(\Omega)}\left\|a_{2}-a_{1}\right\|_{L^{\infty}\left(Q_{R}\right)}\right) .
$$

To prove the result of the lemma it thus suffices to prove that the map $a \mapsto v_{a}$ is continuous from $L^{\infty}\left(0, T_{R} ; \mathbb{R}^{\mathfrak{M}}\right)$ to $L^{2}\left(0, T_{R} ; \mathbb{R}^{\mathfrak{M}}\right)$. This is contained in the proof of Lemma 5.3.

Proof of Lemma 5.9. From Proposition 5.1 and (5.13), (5.16)-(5.17) we have

$$
\begin{aligned}
\left\|y_{R, z}\right\|_{L^{\infty}\left(Q_{R}\right)} & \leqslant h^{-\frac{d}{2}}\left\|y_{R, z}\right\|_{L^{\infty}\left(0, T_{R}, L^{2}(\Omega)\right)} \\
& \leqslant h^{-\frac{d}{2}} e^{C_{1}\left(1+T+\frac{1}{T}+\zeta(R)^{2 / 3}\right)}\left|y_{0}\right|_{L^{2}(\Omega)} .
\end{aligned}
$$

We hence find

$$
R^{-1}\left\|y_{R, z}\right\|_{L^{\infty}\left(Q_{R}\right)} \leqslant h^{-\frac{d}{2}} e^{C_{1}\left(1+T+\frac{1}{T}+\ln ^{\frac{2 r}{3}}(e+R)\right)-\ln (R)}\left|y_{0}\right|_{L^{2}(\Omega)} .
$$

Let $0<\varepsilon<1$ be such that $\alpha=\frac{d}{2(1-\varepsilon)}$. As $r<3 / 2$, there exists $R_{1}=R_{1}(T)>R_{0}$ such that

$$
\zeta(R)^{2 / 3}=K^{2 / 3} \ln ^{\frac{2 r}{3}}(e+R) \geqslant 1+\frac{1}{T},
$$

and

$$
C_{1}\left(1+T+\frac{1}{T}+\ln ^{\frac{2 r}{3}}(e+R)\right)-\ln (R) \leqslant-(1-\varepsilon) \ln (R),
$$

if $R \geqslant R_{1}(T)$, which gives

$$
R^{-1}\left\|y_{R, z}\right\|_{L^{\infty}\left(Q_{R}\right)} \leqslant \frac{h^{-\frac{d}{2}}}{R^{1-\varepsilon}}\left|y_{0}\right|_{L^{2}(\Omega)} .
$$

We set

$$
R=h^{-\alpha} M^{\frac{1}{1-\varepsilon}}=h^{-\frac{d}{2(1-\varepsilon)}} M^{\frac{1}{1-\varepsilon}},
$$

and we have $R \geqslant R_{1}(T)$ by taking $0<h \leqslant \tilde{\tilde{h}}_{2}$ with $\tilde{\tilde{h}}_{2}$ sufficiently small and function of $T$ and $M$. With this choice for $R$ we then obtain

$$
R^{-1}\left\|y_{R, z}\right\|_{L^{\infty}\left(Q_{R}\right)} \leqslant \frac{h^{-\frac{d}{2}}}{R^{1-\varepsilon}}\left|y_{0}\right|_{L^{2}(\Omega)} \leqslant \frac{h^{-\frac{d}{2}}}{R^{1-\varepsilon}} M=1 .
$$

We now recall the sufficient condition (5.18) that connects $R$ and $h$ :

$$
h \leqslant C^{\prime}\left(1+\frac{1}{T}+\zeta(R)^{2 / 3}\right)^{-1} .
$$

By (5.24) as $\zeta(R)^{2 / 3} \geqslant 1+\frac{1}{T}$ if $R \geqslant R_{1}(T)$ it suffices to have

$$
h \leqslant C\left(2 \zeta(R)^{2 / 3}\right)^{-1}, \quad \text { i.e. } R \leqslant e^{\frac{C^{\prime}}{h^{3 / 2 r}}}-e .
$$

Observe that this last condition is satisfied by $R$ as defined in (5.26) for $0<h \leqslant \tilde{h}_{2} \leqslant \tilde{\tilde{h}}_{2}$ with $\tilde{h}_{2}$ taken sufficiently small and function of $T$ and $M$. 


\subsubsection{The one-dimensional case}

Finally, we study the one-dimensional case for which the result of Theorem 5.5 can be sharpened to yield a control function uniformly bounded with respect to the discretization parameter $h$. This require a more regular intial condition which can be achieved by simply setting the control function to zero for a arbitrary small time interval according to the following lemma in the case of an inital condition $y_{0} \in \mathbb{R}^{\mathfrak{M}}$ that lays in a bounded set for the $L^{\infty}$-norm.

Lemma 5.10. Let $y_{0} \in \mathbb{R}^{\mathfrak{M}}$. Consider the homogeneous semi-linear equation

$$
\left(\partial_{t}+\mathcal{A}^{\mathfrak{M}}\right) y+\mathcal{G}(y)=0, \quad t>0, \quad y_{\mid \partial \Omega}=0, \quad y(0)=y_{0} .
$$

There exists $t_{1}>0$, depending on $\left|y_{0}\right|_{L^{\infty}(\Omega)}$, such that the solution exists on $\left[0, t_{1}\right]$ and we have

$$
|y(t)|_{H^{1}(\Omega)} \leqslant C\left(t^{-\frac{1}{2}}\left|y_{0}\right|_{L^{2}(\Omega)}+t^{\frac{1}{2}} \beta\left(\Omega,\left|y_{0}\right|_{L^{\infty}(\Omega)}\right)\right), \quad 0<t \leqslant t_{1},
$$

for some continuous function $\beta$ and some $C>0$ independent of the discretization parameter $h$.

Here, we have introduced the following discrete $H^{1}$-norm:

$$
|u|_{H^{1}(\Omega)}:=|u|_{L^{2}(\Omega)}+\sum_{i \in \llbracket 1, d \rrbracket}\left|D_{i} u\right|_{L^{2}(\Omega)} .
$$

Observe below that the proof of Lemma 5.10 holds in arbitrary dimension.

Proof. For any $h>0$ there exists a unique solution to (5.27) by the Cauchy-Lipschitz theorem and we have the Duhamel formula:

$$
y(t)=S(t) y_{0}+\int_{0}^{t} S(t-s) \mathcal{G}(y(s)) d s,
$$

where $S(t)=e^{-t \mathcal{A}^{\mathfrak{M}}}$. For $s \geqslant 0$, we set $G(s)=\sup _{[-s, s]}|\mathcal{G}|$, which yields a Lipschitz function. By standard properties of the finite-difference scheme, we have $|S(t) u|_{L^{\infty}} \leqslant|u|_{L^{\infty}}$ which gives

$$
\begin{aligned}
|y(t)|_{L^{\infty}} & \leqslant\left|y_{0}\right|_{L^{\infty}}+\int_{0}^{t}|\mathcal{G}(y(s))|_{L^{\infty}} d s \\
& \leqslant\left|y_{0}\right|_{L^{\infty}}+\int_{0}^{t} G\left(|y(s)|_{L^{\infty}}\right) d s .
\end{aligned}
$$

Take $u_{0}>0$ and define

$$
\phi(u)=\int_{u_{0}}^{u} \frac{d v}{G(v)}, \quad u>0 .
$$

The function $\phi$ is increasing and so is its inverse $\phi^{-1}$. The Bihari inequality [5] then yields

$$
|y(t)|_{L^{\infty}} \leqslant \phi^{-1}\left(\phi\left(\left|y_{0}\right|_{L^{\infty}}\right)+t\right), \quad t \in\left[0, t_{1}\right],
$$

with $t_{1}$ chosen sufficiently small and function of $\left|y_{0}\right|_{L^{\infty}}$. This insures the existence of the solution on $\left[0, t_{1}\right]$. Note that $t_{1}$ is chosen independently of $h$. We write

$$
|y(t)|_{L^{\infty}} \leqslant H\left(t,\left|y_{0}\right|_{L^{\infty}}\right) \leqslant H\left(t_{1},\left|y_{0}\right|_{L^{\infty}}\right)=: \mathcal{H}\left(\left|y_{0}\right|_{L^{\infty}}\right), \quad t \in\left[0, t_{1}\right],
$$

as $H$ increases with respect to $t$. 
We now consider the regularization effect. We have $|S(t) u|_{H^{1}} \leqslant C t^{-\frac{1}{2}}|u|_{L^{2}}$ as can be derived using an eigenfunction decomposition. From the Duhamel formula we thus obtain

$$
|y(t)|_{H^{1}} \leqslant C t^{-\frac{1}{2}}\left|y_{0}\right|_{L^{2}}+\int_{0}^{t}(t-s)^{-\frac{1}{2}}|\mathcal{G}(y(s))|_{L^{2}} d s .
$$

As we have $|u|_{L^{2}} \leqslant|\Omega|^{\frac{1}{2}}|u|_{L^{\infty}}$ we obtain

$$
\begin{aligned}
|\mathcal{G}(y(s))|_{L^{2}} & \leqslant|\Omega|^{\frac{1}{2}}|\mathcal{G}(y(s))|_{L^{\infty}} \leqslant|\Omega|^{\frac{1}{2}} G\left(|y(s)|_{L^{\infty}}\right) \\
& \leqslant|\Omega|^{\frac{1}{2}} G\left(\mathcal{H}\left(\left|y_{0}\right|_{L^{\infty}}\right)\right)=: \beta\left(\Omega,\left|y_{0}\right|_{L^{\infty}}\right),
\end{aligned}
$$

for $0<s \leqslant t_{1}$, which gives

$$
\begin{aligned}
|y(t)|_{H^{1}} & \leqslant C t^{-\frac{1}{2}}\left|y_{0}\right|_{L^{2}}+\beta\left(\Omega,\left|y_{0}\right|_{L^{\infty}}\right) \int_{0}^{t}(t-s)^{-\frac{1}{2}} d s \\
& \leqslant C^{\prime}\left(t^{-\frac{1}{2}}\left|y_{0}\right|_{L^{2}}+t^{\frac{1}{2}} \beta\left(\Omega,\left|y_{0}\right|_{L^{\infty}}\right)\right) .
\end{aligned}
$$

The constants are independent of $h$.

We can now state the control result.

Theorem 5.11. Let $d=1$ and $\Omega=(0,1)$ and $\Gamma$ satisfy (1.2). There exists $C_{0}$ such that, for $T>0$ and $M>0$, there exist positive constants $C, h_{3}>0$ such that for $0<h \leqslant h_{3}$ and for all initial data $y_{0} \in \mathbb{R}^{\mathfrak{M}}$ satisfying $\left|y_{0}\right|_{H^{1}(\Omega)} \leqslant M$, there exists a semi-discrete control function $v$ such that the solution to the semi-linear parabolic equation

$$
\left(\partial_{t}+\mathcal{A}^{\mathfrak{M}}\right) y+\mathcal{G}(y)=\mathbf{1}_{\omega} v, \quad y_{\mid \partial \Omega}=0, \quad y(0)=y_{0}
$$

satisfies

$$
|y(T)|_{L^{2}(\Omega)} \leqslant C e^{-C_{0} / h}\left|y_{0}\right|_{L^{2}(\Omega)}, \quad \text { and } \quad\|v\|_{L^{2}(Q)} \leqslant C\left|y_{0}\right|_{L^{2}(\Omega)} .
$$

Here $C=C(T, M)$.

Proof. The proof follows that of Theorem 5.5. We set $Z_{R}=L^{\infty}\left(0, T_{R} ; \mathbb{R}^{\mathfrak{M}}\right)$. Denoting by $B_{R}$ the ball centered at 0 and of radius $R$ in $Z_{R}$, the following lemma replaces Lemma 5.9.

Lemma 5.12. There exists $R_{0}=R_{0}(T, M)$ such that the map $\Lambda_{R}$ maps $B_{R}$ into itself if $R \geqslant R_{0}$ and if $\left|y_{0}\right|_{H^{1}(\Omega)} \leqslant M$.

Remark 5.13. Lemma 5.12 is the result leading to the improvement of the controllability result as compared to higher dimensions. Its proof below is the only occurrence of the assumption that $d=1$.

Here $R_{0}$ is not connected to $h$. We choose $R=R_{0}(T, M)$. If we take $h$ sufficiently small, $0<h \leqslant \tilde{h}_{3}$ with $\tilde{h}_{3}=$ $\tilde{h}_{3}(T, M)=\min \left(h_{0}, C\left(1+1 / T+\zeta(R)^{2 / 3}\right)^{-1}\right)$, then (5.18) is fulfilled.

As $\Lambda_{R}$ is also continuous and $\Lambda_{R}\left(B_{R}\right)$ is relatively compact this yields the existence of $y \in B_{R}$ such that $\Lambda_{R}(y)=y$. Setting $v_{R}=L_{T_{R}, a_{y}}\left(y_{0}\right)$ we obtain

$$
\left(\partial_{t}+\mathcal{A}^{\mathfrak{M}}\right) y+y a_{y}=\mathbf{1}_{\omega} v_{R}, \quad t \in\left(0, T_{R}\right], \quad y_{\mid \partial \Omega}=0, \quad y(0)=y_{0} .
$$

Since $y \in B_{R}$ we have $\|y\|_{L^{\infty}(Q)} \leqslant R$. Then we have $a_{y}=g\left(T_{R}(y)\right)=g(y)$, which yields

$$
\left(\partial_{t}+\mathcal{A}^{\mathfrak{M}}\right) y+\mathcal{G}(y)=\mathbf{1}_{\omega} v_{R}, \quad t \in\left(0, T_{R}\right], \quad y_{\mid \partial \Omega}=0, \quad y(0)=y_{0} .
$$


With the estimations (5.15)-(5.16) we have

$$
\left|y\left(T_{R}\right)\right|_{L^{2}(\Omega)} \leqslant C(T, M) e^{-C_{0} / h}\left|y_{0}\right|_{L^{2}(\Omega)}, \quad\left\|v_{R}\right\|_{L^{2}\left(Q_{R}\right)} \leqslant C(T, M)\left|y_{0}\right|_{L^{2}(\Omega)},
$$

as $R=R_{0}(T, M)$ is chosen independently of $h$ here.

We now define $v$ on $[0, T]$ by

$$
v= \begin{cases}v_{R} & \text { if } 0<t \leqslant T_{R}, \\ 0 & \text { if } T_{R}<t \leqslant T .\end{cases}
$$

We naturally have $\|v\|_{L^{2}(Q)} \leqslant C(T, M)\left|y_{0}\right|_{L^{2}(\Omega)}$.

If we have

$$
h^{-1 / 2}\left|y\left(T_{R}\right)\right|_{L^{2}(\Omega)} \leqslant M_{0},
$$

we can apply Proposition 5.4 on the time interval $\left[T_{R}, T\right]$, which yields

$$
|y(T)|_{L^{2}(\Omega)} \leqslant\left|y\left(T_{R}\right)\right|_{L^{2}(\Omega)} e^{K_{0}\left(T-T_{R}\right)} \leqslant C(T, M) e^{-C_{0} / h}\left|y_{0}\right|_{L^{2}(\Omega)} .
$$

With (5.29), choosing $h_{3} \leqslant \min \left(h_{0}, \tilde{h}_{3}\right)$ sufficiently small, condition (5.30) can be fulfilled if $0<h \leqslant h_{3}$, which concludes the proof.

Proof of Lemma 5.12. From Proposition 5.1 and (5.13) we have

$$
\begin{aligned}
\left\|D y_{R, z}\right\|_{L^{\infty}\left(0, T_{R}, L^{2}(\Omega)\right)} & \leqslant e^{C\left(1+T_{R}+\left(T_{R}^{\frac{1}{2}}+T_{R}\right)\left\|a_{z}\right\|_{\infty}\right)}\left(\left|D y_{0}\right|_{L^{2}(\Omega)}+\|v\|_{L^{2}(Q)}\right) \\
& \leqslant e^{C\left(1+T_{R}+\frac{1}{T_{R}}+\left(T_{R}^{\frac{1}{2}}+T_{R}\right)\left\|a_{z}\right\|_{\infty}+\left\|a_{z}\right\|_{\infty}^{2 / 3}\right)}\left|y_{0}\right|_{H^{1}(\Omega)} \\
& \leqslant e^{C^{\prime}\left(1+T+\frac{1}{T}+\zeta(R)^{2 / 3}\right)}\left|y_{0}\right|_{H^{1}(\Omega)} .
\end{aligned}
$$

In the one-dimensional case if $f \in \mathbb{R}^{\mathfrak{M}}$ with $f_{\mid \partial \Omega}=0$ we have

$$
|f|_{L^{\infty}} \leqslant|D f|_{L^{1}(\Omega)} \leqslant C_{\Omega}|D f|_{L^{2}(\Omega)} .
$$

We emphasize here that this classical Sobolev embedding is the only place where the assumption $d=1$ is used.

We thus obtain

$$
\left\|y_{R, z}\right\|_{L^{\infty}\left(Q_{R}\right)} \leqslant e^{C\left(1+T+\frac{1}{T}+\zeta(R)^{2 / 3}\right)}\left|y_{0}\right|_{H^{1}(\Omega)} .
$$

We hence find

$$
R^{-1}\left\|y_{R, z}\right\|_{L^{\infty}\left(Q_{R}\right)} \leqslant e^{C\left(1+T+\frac{1}{T}+\ln ^{2 r / 3}(e+R)\right)-\ln (R)}\left|y_{0}\right|_{H^{1}(\Omega)} .
$$

As $r<3 / 2$, if $\left|y_{0}\right|_{H^{1}(\Omega)} \leqslant M$ there exists $R_{0}>0$, depending on $T$ and $M$ such that

$$
\left\|y_{R, z}\right\|_{L^{\infty}\left(Q_{R}\right)} \leqslant R, \quad \text { if } R \geqslant R_{0} .
$$

Hence, for $R \geqslant R_{0}$ the map $\Lambda_{R}$ maps $B_{R}$ into itself.

Remark 5.14 (Local controllability in one space dimension). Estimate (5.31) is used above to prove controllability thanks to the form of the non-linearity. For an arbitrary non-linearity one can also use (5.31) and impose a sufficiently small initial condition $y_{0}$ in $H^{1}$-norm, which yields

$$
\left\|y_{R, z}\right\|_{L^{\infty}\left(Q_{R}\right)} \leqslant R .
$$

The rest of the proof remains unchanged and this yields the following local controllability result. 
Theorem 5.15. Let $d=1$ and $\Omega=(0,1), \Gamma$ satisfy (1.2), and the function $\mathcal{G}$ of the form (5.4). There exists $C_{0}$ such that, for $T>0$ there exist positive constants $C, h_{3}>0$ and $\varepsilon>0$, such that for $0<h \leqslant h_{3}$ and for all initial data $y_{0} \in \mathbb{R}^{\mathfrak{M}}$ satisfying $\left|y_{0}\right|_{H^{1}(\Omega)} \leqslant \varepsilon$, there exists a semi-discrete control function $v$ such that the solution to the semi-linear parabolic equation

$$
\left(\partial_{t}+\mathcal{A}^{\mathfrak{M}}\right) y+\mathcal{G}(y)=\mathbf{1}_{\omega} v, \quad y_{\mid \partial \Omega}=0, \quad y(0)=y_{0} .
$$

satisfies

$$
|y(T)|_{L^{2}(\Omega)} \leqslant C e^{-C_{0} / h}\left|y_{0}\right|_{L^{2}(\Omega)}, \quad \text { and } \quad\|v\|_{L^{2}(Q)} \leqslant C\left|y_{0}\right|_{L^{2}(\Omega)} .
$$

Remark 5.16. Smallness of the initial condition in $H^{1}$-norm can be obtained by setting the control function to zero for a short initial time and starting from a small initial condition in $L^{2}$-norm that also lays in a bounded set of $L^{\infty}$-norm (see Lemma 5.10).

\section{Acknowledgements}

The authors wish to thank an anonymous referee for a very detailed reading of the article and valuable comments and suggestions.

\section{Appendix A. Proofs of intermediate results in Section 3}

\section{A.1. Proof of Lemma 3.4}

We have

$$
I_{13}=\sum_{i \in \llbracket 1, d \rrbracket} \iint_{Q} r \overline{\tilde{\rho}}^{i} \bar{D}_{i}\left(\gamma_{i} D_{i} v\right) \partial_{t} v d t .
$$

As $\left.v\right|_{\partial \Omega}=0$, with a discrete integration by parts, we have

$$
I_{13}=-\sum_{i \in \llbracket 1, d \rrbracket} \iint_{Q} D_{i}\left(r \overline{\tilde{\rho}}^{i} \partial_{t} v\right) \gamma_{i} D_{i} v d t=Q_{1}+Q_{2},
$$

with

$$
\begin{aligned}
& Q_{1}=-\sum_{i \in \llbracket 1, d \rrbracket} \iint_{Q} D_{i}\left(r \overline{\tilde{\rho}}^{i}\right) \partial_{t} \tilde{v}^{i} \gamma_{i} D_{i} v d t, \\
& Q_{2}=-\sum_{i \in \llbracket 1, d \rrbracket} \iint_{Q} r{\widetilde{\tilde{\rho}^{i}}}^{i}\left(\partial_{t} D_{i} v\right) \gamma_{i} D_{i} v d t .
\end{aligned}
$$

Proposition 2.13 shows that $D_{i}\left(r \overline{\tilde{\rho}}^{i}\right)=\mathcal{O}_{\lambda, \mathfrak{K}}(s h)$, it then follows that

$$
\begin{aligned}
\left|Q_{1}\right| & \leqslant \sum_{i \in \llbracket 1, d \rrbracket} \iint_{Q} s^{-1} \mathcal{O}_{\lambda, \mathfrak{K}}(s h)\left(\partial_{t} \tilde{v}^{i}\right)^{2} d t+\sum_{i \in \llbracket 1, d \rrbracket} \iint_{Q} s \mathcal{O}_{\lambda, \mathfrak{K}}(s h)\left(D_{i} v\right)^{2} d t \\
& \leqslant \sum_{i \in \llbracket 1, d \rrbracket} \iint_{Q} s^{-1} \mathcal{O}_{\lambda, \mathfrak{K}}(s h) \widetilde{\left(\partial_{t} v\right)^{2}} d t+\sum_{i \in \llbracket 1, d \rrbracket} \iint_{Q} s \mathcal{O}_{\lambda, \mathfrak{K}}(s h)\left(D_{i} v\right)^{2} d t \\
& =\iint_{Q} s^{-1} \mathcal{O}_{\lambda, \mathfrak{K}}(s h)\left(\partial_{t} v\right)^{2} d t+\sum_{i \in \llbracket 1, d \rrbracket} \iint_{Q} s \mathcal{O}_{\lambda, \mathfrak{K}}(s h)\left(D_{i} v\right)^{2} d t
\end{aligned}
$$

as $\left(\partial_{t} \tilde{v}^{i}\right)^{2} \leqslant{\widetilde{\left(\partial_{t} v\right)^{2}}}^{i}$, by convexity and as $\left.v\right|_{\partial \Omega}=0$. 
We write, using that $\gamma_{i}$ do not depend on time, that

$$
\begin{aligned}
& Q_{2}=-\frac{1}{2} \sum_{i \in \llbracket 1, d \rrbracket} \iint_{Q} r{\widetilde{\tilde{\rho}^{i}}}^{i} \gamma_{i} \partial_{t}\left(D_{i} v\right)^{2} d t \\
& =\frac{1}{2} \sum_{i \in \llbracket 1, d \rrbracket} \iint_{Q} \partial_{t}\left(r \widetilde{\tilde{\tilde{\rho}}}^{i}{ }^{i}\right) \gamma_{i}\left(D_{i} v\right)^{2} d t-\left.\frac{1}{2} \sum_{i \in \llbracket 1, d \rrbracket} \int_{\Omega} r{\widetilde{\tilde{\rho}^{i}}}^{i} \gamma_{i}\left(D_{i} v\right)^{2}\right|_{t=0} ^{t=T} .
\end{aligned}
$$

We observe that for $0<s h<\varepsilon_{1}(\lambda)$ with $\varepsilon_{1}(\lambda)$ sufficiently small we have $r \overline{\tilde{\rho}}^{i}>0$ by Proposition 2.13. The signs of the terms at $t=T$ and $t=0$ are thus prescribed. Moreover, by Proposition 2.14, we know that $\partial_{t}\left(r \overline{\tilde{\rho}}^{i}\right)=$ $T(s h)^{2} \theta \mathcal{O}_{\lambda, \mathfrak{K}}(1)$ so that, for $s h \leqslant \mathfrak{K}$, we obtained the result.

\section{A.2. Proof of Lemma 3.7}

We have

$$
I_{23}=\sum_{i \in \llbracket 1, d \rrbracket} \iint_{Q} \gamma_{i} r\left(\bar{D}_{i} D_{i} \rho\right) \overline{\tilde{v}}^{i} \partial_{t} v d t
$$

As $\left.v\right|_{\partial \Omega}=0$ we write

$$
I_{23}=\sum_{i \in \llbracket 1, d \rrbracket} \iint_{Q}{\widehat{\gamma_{i} r\left(\bar{D}_{i} D_{i} \rho\right) \partial_{t} v}}^{i} \tilde{v}^{i} d t=Q_{1}+Q_{2},
$$

by Lemma 2.2 with

$$
\begin{aligned}
Q_{1} & =\sum_{i \in \llbracket 1, d \rrbracket} \iint_{Q}{\overline{\gamma_{i} r\left(\bar{D}_{i} D_{i} \rho\right)}}^{i} \partial_{t} \tilde{v}^{i} \tilde{v}^{i} d t, \\
Q_{2} & =\sum_{i \in \llbracket 1, d \rrbracket} \frac{h_{i}^{2}}{4} \iint_{Q} D_{i}\left(\gamma_{i} r\left(\bar{D}_{i} D_{i} \rho\right)\right)\left(D_{i} \partial_{t} v\right) \tilde{v}^{i} d t .
\end{aligned}
$$

We have

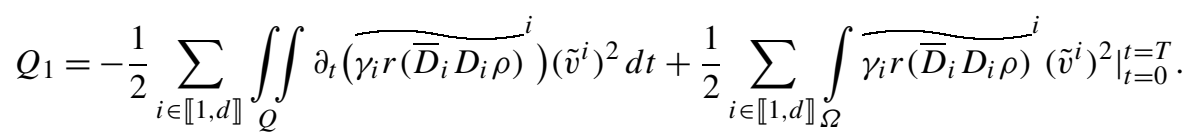

By Proposition 2.13 and Lemma 2.7 we have

$$
{\overline{\gamma_{i} r\left(\bar{D}_{i} D_{i} \rho\right)}}^{i}=s^{2} \mathcal{O}_{\lambda, \mathfrak{K}}(1)
$$

Lemma A.1. We have

$$
\partial_{t}\left({\overline{\gamma_{i} r\left(\bar{D}_{i} D_{i} \rho\right)}}^{i}\right)=T s^{2} \theta \mathcal{O}_{\lambda, \mathfrak{K}}(1) .
$$

Proof. Since $\gamma_{i}$ do not depend on time, we have

$$
\partial_{t}\left(\gamma_{i} r\left(\bar{D}_{i} D_{i} \rho\right)\right)=\gamma_{i} \partial_{t}\left(r\left(\bar{D}_{i} D_{i} \rho\right)\right)
$$

which is bounded by $T s^{2} \theta \mathcal{O}_{\lambda, \mathfrak{K}}(1)$ thanks to Proposition 2.14. The action of the avering operator ${ }^{-i}$ does not affect the form of this estimate.

As $\left(\tilde{v}^{i}\right)^{2} \leqslant{\widetilde{(v)^{2}}}^{i}$ and $\left.v\right|_{\partial \Omega}=0$, we thus have 


$$
Q_{1}=\iint_{Q} T s^{2} \theta \mathcal{O}_{\lambda, \mathfrak{K}}(1) v^{2} d t+\int_{\Omega} s^{2}\left(\mathcal{O}_{\lambda, \mathfrak{K}}(1) v_{\mid t=T}^{2}+\mathcal{O}_{\lambda, \mathfrak{K}}(1) v_{\mid t=0}^{2}\right) .
$$

With an integration by parts in time and Lemma 2.1 we obtain $Q_{2}=Q_{a}+Q_{b}$, with

$$
\begin{aligned}
Q_{a} & =-\sum_{i \in \llbracket 1, d \rrbracket} \frac{h_{i}^{2}}{4} \iint_{Q} \partial_{t}\left(D_{i}\left(\gamma_{i} r\left(\bar{D}_{i} D_{i} \rho\right)\right) \tilde{v}^{i}\right)\left(D_{i} v\right) d t \\
Q_{b} & =\left.\sum_{i \in \llbracket 1, d \rrbracket} \frac{h_{i}^{2}}{8} \int_{\Omega} D_{i}\left(\gamma_{i} r\left(\bar{D}_{i} D_{i} \rho\right)\right) D_{i}\left(v^{2}\right)\right|_{t=0} ^{t=T} .
\end{aligned}
$$

With Lemma 2.1 and as $\left.v\right|_{\partial \Omega}=0$ with a discrete integration by parts in space (Proposition 2.4) we have

$$
\begin{aligned}
Q_{a}= & \sum_{i \in \llbracket 1, d \rrbracket} \frac{h_{i}^{2}}{8} \iint_{Q}\left(\partial_{t} \bar{D}_{i} D_{i}\left(\gamma_{i} r\left(\bar{D}_{i} D_{i} \rho\right)\right)\right) v^{2} d t \\
& -\sum_{i \in \llbracket 1, d \rrbracket} \frac{h_{i}^{2}}{4} \iint_{Q}\left(D_{i}\left(\gamma_{i} r\left(\bar{D}_{i} D_{i} \rho\right)\right)\right)\left(\partial_{t} \tilde{v}^{i}\right)\left(D_{i} v\right) d t .
\end{aligned}
$$

To estimate $Q_{b}$ we perform a discrete integration by parts using that $\left.v\right|_{\partial \Omega}=0$,

$$
Q_{b}=-\left.\sum_{i \in \llbracket 1, d \rrbracket} \frac{h_{i}^{2}}{8} \int_{\Omega} \bar{D}_{i} D_{i}\left(\gamma_{i} r\left(\bar{D}_{i} D_{i} \rho\right)\right) v^{2}\right|_{t=0} ^{t=T} .
$$

Lemma A.2. We have

$$
\begin{aligned}
& D_{i}\left(\gamma_{i} r\left(\bar{D}_{i} D_{i} \rho\right)\right)=s^{2} \mathcal{O}_{\lambda, \mathfrak{K}}(1), \quad h_{i}^{2} \bar{D}_{i} D_{i}\left(\gamma_{i} r\left(\bar{D}_{i} D_{i} \rho\right)\right)=s(s h) \mathcal{O}_{\lambda, \mathfrak{K}}(1), \\
& h_{i}^{2} \partial_{t} \bar{D}_{i} D_{i}\left(\gamma_{i} r\left(\bar{D}_{i} D_{i} \rho\right)\right)=T s^{2} \theta \mathcal{O}_{\lambda, \mathfrak{K}}(1) .
\end{aligned}
$$

Proof. We use Lemma 2.1 to obtain

$$
D_{i}\left(\gamma_{i} r\left(\bar{D}_{i} D_{i} \rho\right)\right)=\left(D_{i} \gamma_{i}\right){\overline{r\left(\bar{D}_{i} D_{i} \rho\right)}}^{i}+\tilde{\gamma}_{i}^{i} D_{i}\left(r\left(\bar{D}_{i} D_{i} \rho\right)\right)
$$

and the required estimate follows from the Lipschitz regularity of $\gamma_{i}$ and Proposition 2.13. The second estimate is deduced from the first one by observing that $h_{i} D_{i}=\tau_{i}^{+}-\tau_{i}^{-}$. For the third estimate, since $\gamma_{i}$ do not depend on the time, we can write

$$
h_{i}^{2} \partial_{t} \bar{D}_{i} D_{i}\left(\gamma_{i} r\left(\bar{D}_{i} D_{i} \rho\right)\right)=\left(\bar{\tau}_{i}^{+}-\bar{\tau}_{i}^{-}\right)\left(\tau_{i}^{+}-\tau_{i}^{-}\right)\left(\gamma_{i} \partial_{t}\left(r\left(\bar{D}_{i} D_{i} \rho\right)\right)\right) .
$$

The conclusion comes from Proposition 2.14.

With the Cauchy-Schwarz inequality, using that $\left(\partial_{t} \tilde{v}^{i}\right)^{2} \leqslant{\widetilde{\left(\partial_{t} v\right)^{2}}}^{i}$ and $\left.v\right|_{\partial \Omega}=0$ we obtain

$$
\begin{aligned}
Q_{a} \geqslant & \iint_{Q} T s^{2} \theta \mathcal{O}_{\lambda, \mathfrak{K}}(1)(v)^{2} d t+\iint_{Q}(s h)^{2} s^{-1} \mathcal{O}_{\lambda, \mathfrak{K}}(1)\left(\partial_{t} v\right)^{2} d t \\
& +\sum_{i \in \llbracket 1, d \rrbracket} \iint_{Q}(s h)^{2} s \mathcal{O}_{\lambda, \mathfrak{K}}(1)\left(D_{i} v\right)^{2} d t .
\end{aligned}
$$

and

$$
Q_{b}=\left.\int_{\Omega} \mathcal{O}_{\lambda, \mathfrak{K}}(1)\left(s(s h) v^{2}\right)\right|_{t=T}+\left.\int_{\Omega} \mathcal{O}_{\lambda, \mathfrak{K}}(1)\left(s(s h) v^{2}\right)\right|_{t=0} .
$$

In fine, collecting (A.2), (A.3), (A.4), we obtain the result. 


\section{A.3. Proof of Lemma 3.8}

We have

$$
I_{31}=-2 \tau \sum_{i \in \llbracket 1, d \rrbracket} \iint_{Q}\left(\partial_{t} \theta\right) \varphi \gamma_{i} r{\overline{D_{i} \rho}}^{i} v{\overline{D_{i} v}}^{i} d t .
$$

As $\left.v\right|_{\partial \Omega}=0$, we write

$$
I_{31}=-2 \tau \sum_{i \in \llbracket 1, d \rrbracket} \iint_{Q}\left(\partial_{t} \theta\right){\overline{\varphi \gamma_{i} r \bar{D}_{i} \rho}}^{i} v D_{i} v d t
$$

We have

$$
{\overline{\varphi \gamma_{i} r{\overline{D_{i} \rho}}^{i} v}}^{i}={\overline{\varphi \gamma_{i} r{\overline{D_{i} \rho}}^{i}}}^{i} \tilde{v}^{i}+\frac{h_{i}^{2}}{4} D_{i}\left(\varphi \gamma_{i} r{\overline{D_{i} \rho}}^{i}\right) D_{i} v
$$

We obtain

$$
\begin{aligned}
I_{31} & =-\tau \sum_{i \in \llbracket 1, d \rrbracket} \iint_{Q}\left(\partial_{t} \theta\right) \overline{\varphi \gamma_{i} r{\overline{D_{i} \rho}}^{i}} D_{i} D_{i}\left(v^{2}\right) d t-\frac{h_{i}^{2}}{2} \tau \sum_{i \in \llbracket 1, d \rrbracket} \iint_{Q}\left(\partial_{t} \theta\right) D_{i}\left(\varphi \gamma_{i} r{\overline{D_{i} \rho}}^{i}\right)\left(D_{i} v\right)^{2} d t \\
& \left.=\tau \sum_{i \in \llbracket 1, d \rrbracket} \iint_{Q}\left(\partial_{t} \theta\right){\overline{D_{i}\left(\varphi \gamma_{i} r{\overline{D_{i}}}^{i}\right.}}^{i}\right)^{i} v^{2} d t-\frac{h_{i}^{2}}{2} \tau \sum_{i \in \llbracket 1, d \rrbracket} \iint_{Q}\left(\partial_{t} \theta\right) D_{i}\left(\varphi \gamma_{i} r{\overline{D_{i} \rho}}^{i}\right)\left(D_{i} v\right)^{2} d t,
\end{aligned}
$$

with a discrete integration by parts.

By using the Lipschitz continuity of $\varphi \gamma_{i}$ and Proposition 2.13 we obtain

$$
D_{i}\left(\varphi \gamma_{i} r{\overline{D_{i} \rho}}^{i}\right)=s \mathcal{O}_{\lambda, \mathfrak{K}}(1), \quad{\overline{D_{i}\left(\varphi \gamma_{i} r{\overline{D_{i} \rho}}^{i}\right.}}^{i}=s \mathcal{O}_{\lambda, \mathfrak{K}}(1) .
$$

With (3.1), the result follows.

\section{A.4. Proof of Lemma 3.9}

We have

$$
\begin{aligned}
I_{33} & =-\tau \iint_{Q}\left(\partial_{t} \theta\right) \varphi v \partial_{t} v d t=-\frac{1}{2} \tau \iint_{Q}\left(\partial_{t} \theta\right) \varphi \partial_{t}\left(v^{2}\right) d t \\
& =\frac{1}{2} \tau \iint_{Q}\left(\partial_{t}^{2} \theta\right) \varphi v^{2} d t-\left.\frac{1}{2} \tau \int_{\Omega}\left(\partial_{t} \theta\right) \varphi v^{2}\right|_{t=0} ^{t=T} .
\end{aligned}
$$

With (3.1) we have

$$
-\partial_{t} \theta(0)=\partial_{t} \theta(T)=T \theta^{2}(T)>0 .
$$

As $\varphi<0$ the result follows.

\section{A.5. Proof of Lemma 3.11}

We choose $i, j \in \llbracket 1, d \rrbracket$ with $i \neq j$. We have

$$
\iint_{Q} s \phi \gamma_{i}\left(D_{i} v\right)^{2} d t
$$




$$
\begin{aligned}
& \geqslant C \iint_{Q} s \phi\left(D_{i} v\right)^{2} d t=C \iint_{Q} s{\widehat{\phi\left(D_{i} v\right)^{2}}}^{j} d t \\
& =C \iint_{Q} s \tilde{\phi}^{j}{\widetilde{\left(D_{i} v\right)^{2}}}^{j} d t+C \frac{h^{2}}{4} \iint_{Q} s\left(D_{j} \phi\right) D_{j}\left(D_{i} v\right)^{2} d t \\
& =C \iint_{Q} s \tilde{\phi}^{j}\left({\widetilde{D_{i}} v^{j}}^{j} d t+C \frac{h^{2}}{4} \iint_{Q} s \tilde{\phi}^{j}\left(D_{j} D_{i} v\right)^{2} d t-C \frac{h^{2}}{4} \iint_{Q} s\left(\bar{D}_{j} D_{j} \phi\right)\left(D_{i} v\right)^{2} d t,\right.
\end{aligned}
$$

by Proposition 2.4 as $\left.D_{i} v\right|_{(0, T) \times \partial_{j} \Omega}=0$ and by Lemma 2.2. We thus have

$$
\iint_{Q} s \phi \gamma_{i}\left(D_{i} v\right)^{2} d t \geqslant C \frac{h^{2}}{4} \iint_{Q} s \tilde{\phi}^{j}\left(D_{j} D_{i} v\right)^{2} d t-C \frac{h^{2}}{4} \iint_{Q} s\left(\bar{D}_{j} D_{j} \phi\right)\left(D_{i} v\right)^{2} d t .
$$

With Lemma 2.6 we note that

$$
\tilde{\phi}^{j}=\phi+h^{2} \mathcal{O}_{\lambda}(1), \quad \bar{D}_{j} D_{j} \phi=\partial_{j}^{2} \phi+h^{2} \mathcal{O}_{\lambda}(1)=\mathcal{O}_{\lambda}(1),
$$

which justifies the last term in $H$, and contributes to the first term in $\tilde{X}$ and the first term in $\tilde{W}$.

Similarly for $i \in \llbracket 1, d \rrbracket$, we also write

$$
\begin{aligned}
\iint_{Q} \gamma_{i} \phi\left(D_{i} v\right)^{2} d t & \geqslant C \iint_{Q} \phi\left(D_{i} v\right)^{2} d t \\
& =\underbrace{\iint_{Q} \overline{\phi\left(D_{i} v\right)^{2}}}_{=\mathscr{Q}_{i}}{ }^{i} d t+\frac{h_{i}}{2} \iint_{Q_{i}}\left(\left(\phi\left(D_{i} v\right)^{2}\right)_{\frac{1}{2}}+\left(\phi\left(D_{i} v\right)^{2}\right)_{N_{i}+\frac{1}{2}}\right) d t,
\end{aligned}
$$

by Proposition 2.4, and Lemma 2.2 yields

$$
\begin{aligned}
\mathscr{Q}_{i}= & \iint_{Q} \bar{\phi}^{i}{\overline{\left(D_{i} v\right)^{2}}}^{i} d t+\frac{h_{i}^{2}}{4} \iint_{Q}\left(\bar{D}_{i} \phi\right) \bar{D}_{i}\left(D_{i} v\right)^{2} d t \\
= & \iint_{Q} \bar{\phi}^{i}\left({\overline{D_{i}} v}^{i}\right)^{2} d t+\frac{h_{i}^{2}}{4} \iint_{Q} \bar{\phi}^{i}\left(\bar{D}_{i} D_{i} v\right)^{2} d t-\frac{h_{i}^{2}}{4} \iint_{Q}\left(D_{i} \bar{D}_{i} \phi\right)\left(D_{i} v\right)^{2} d t \\
& +\frac{h_{i}^{2}}{4} \iint_{Q_{i}}\left(\left(\bar{D}_{i} \phi\right)_{N_{i}+1}\left(D_{i} v\right)_{N_{i}+\frac{1}{2}}^{2}-\left(\bar{D}_{i} \phi\right)_{0}\left(D_{i} v\right)_{\frac{1}{2}}^{2}\right) d t
\end{aligned}
$$

We observe that

$$
\frac{h_{i}}{2} \iint_{Q_{i}}\left(\left(\phi\left(D_{i} v\right)^{2}\right)_{\frac{1}{2}}+\left(\phi\left(D_{i} v\right)^{2}\right)_{N_{i}+\frac{1}{2}}\right) d t+\frac{h_{i}^{2}}{4} \iint_{Q_{i}}\left(\left(\bar{D}_{i} \phi\right)_{N_{i}+1}\left(D_{i} v\right)_{N_{i}+\frac{1}{2}}^{2}-\left(\bar{D}_{i} \phi\right)_{0}\left(D_{i} v\right)_{\frac{1}{2}}^{2}\right) d t
$$

can be made non-negative for $h$ sufficiently small once $\lambda$ is fixed, as $\bar{D}_{i} \phi=\mathcal{O}_{\lambda}(1)$. With Lemma 2.6 we note that

$$
\tilde{\phi}^{i}=\phi+h \mathcal{O}_{\lambda}(1), \quad D_{i} \bar{D}_{i} \phi=\partial_{i}^{2} \phi+h^{2} \mathcal{O}_{\lambda}(1)=\mathcal{O}_{\lambda}(1),
$$

which justifies the first and second term in $H$, and contributes to the two terms in $\tilde{X}$ and the second term in $\tilde{W}$. 


\section{A.6. Proof of Lemma 3.12}

We adapt the procedure followed in the continuous case (see e.g. $[12,9,17])$. We multiply the equation satisfied by $u$, i.e. $P^{\mathfrak{M}} u=f$, by $s r^{2} \chi u$, where $\chi \in \mathscr{C}_{c}^{\infty}(\omega)$ is such that $\chi \geqslant 0$ and $\chi=1$ in a neighborhood of $\omega_{1}$. We then integrate over $Q$ :

$$
\iint_{Q} s r^{2} \chi u \partial_{t} u d t-\sum_{i \in \llbracket 1, d \rrbracket} \iint_{Q} s r^{2} \chi u \bar{D}_{i}\left(\gamma_{i} D_{i} u\right) d t=\iint_{Q} s r^{2} \chi u f d t .
$$

We first note that the r.h.s. of (A.6) can be estimated by

$$
\left|\iint_{Q} s r^{2} \chi u f d t\right| \leqslant C\|r f\|_{L^{2}(Q)}^{2}+C\|s r u\|_{L^{2}((0, T) \times \omega)}^{2} .
$$

For the first term in the 1.h.s. of (A.6) we write

$$
\begin{aligned}
\iint_{Q} s r^{2} \chi u \partial_{t} u d t & =\frac{1}{2} \iint_{Q} s r^{2} \chi \partial_{t}\left(u^{2}\right) d t \\
& =-\frac{1}{2} \tau \iint_{Q} \partial_{t}\left(\theta r^{2}\right) \chi u^{2} d t+\left.\frac{1}{2} \int s r_{\Omega}^{2} \chi u^{2}\right|_{t=0} ^{t=T},
\end{aligned}
$$

with an integration by parts w.r.t. $t$. Writing $\partial_{t}\left(\theta r^{2}\right)=\theta^{\prime}(1+2 s \varphi) r^{2}$ and using (3.1) we find, for $s$ large,

$$
\left|\partial_{t}\left(\theta r^{2}\right)\right| \leqslant C T \theta^{2} s r^{2} \leqslant C^{\prime} \theta s^{2} r^{2}
$$

as $\tau \geqslant \tau_{1} T$. We thus find

$$
\left|\iint_{Q} s r^{2} \chi u \partial_{t} u d t\right| \leqslant C\left(\left\|s^{\frac{3}{2}} r u\right\|_{L^{2}((0, T) \times \omega)}^{2}+h^{-1}\left|r u_{\mid t=0}\right|_{L^{2}(\Omega)}^{2}+h^{-1}\left|r u_{\mid t=T}\right|_{L^{2}(\Omega)}^{2}\right),
$$

as $s h \leqslant \varepsilon_{0}$.

In the second term in the 1.h.s. of (A.6) we perform a discrete integration by parts to yield

$$
\begin{aligned}
-\sum_{i \in \llbracket 1, d \rrbracket} \iint_{Q} s r^{2} \chi u \bar{D}_{i}\left(\gamma_{i} D_{i} u\right) & =\sum_{i \in \llbracket 1, d \rrbracket} \iint_{Q} s D_{i}\left(r^{2} \chi u\right) \gamma_{i} D_{i} u \\
& =\sum_{i \in \llbracket 1, d \rrbracket} \iint_{Q} s \gamma_{i} \widetilde{r}^{2} \chi^{i}\left(D_{i} u\right)^{2}+\sum_{i \in \llbracket 1, d \rrbracket} \iint_{Q} s \gamma_{i} D_{i}\left(r^{2} \chi\right) \tilde{u}^{i} D_{i} u .
\end{aligned}
$$

For the first term in the r.h.s. of (A.8), for $s h$ sufficiently small, by Proposition 2.10, we have

$$
\sum_{i \in \llbracket 1, d \rrbracket} \iint_{Q} s \gamma_{i} \widetilde{r}^{2} \chi^{i}\left(D_{i} u\right)^{2} \geqslant C \sum_{i \in \llbracket 1, d \rrbracket}\left\|s^{\frac{1}{2}} r D_{i} u\right\|_{L^{2}\left((0, T) \times \omega_{1}\right)}^{2}
$$

For the second term in the r.h.s. of (A.8) we write

$$
\begin{aligned}
& \sum_{i \in \llbracket 1, d \rrbracket} \iint_{Q} s \gamma_{i} D_{i}\left(r^{2} \chi\right) \tilde{u}^{i} D_{i} u \\
& \quad=\sum_{i \in \llbracket 1, d \rrbracket} \iint_{Q} s \gamma_{i}{\widetilde{r^{2}}}^{i} D_{i}(\chi) \tilde{u}^{i} D_{i} u+\frac{1}{2} \sum_{i \in \llbracket 1, d \rrbracket} \iint_{Q} s \gamma_{i} D_{i}\left(r^{2}\right) \tilde{\chi}^{i} D_{i}\left(u^{2}\right) .
\end{aligned}
$$

The first term in the r.h.s. of (A.10) can be estimated by

$$
\left|\iint_{Q} s \gamma_{i}{\widetilde{r^{2}}}^{i} D_{i}(\chi) \tilde{u}^{i} D_{i} u\right| \leqslant C\left\|r D_{i} u\right\|_{L^{2}(Q)}^{2}+C\|s r u\|_{L^{2}((0, T) \times \omega)}^{2},
$$


for $h$ and $s h$ sufficiently small, as $\operatorname{supp}(\chi) \Subset \omega$. For the second term in the r.h.s. of (A.10) a discrete integration by parts yields

$$
\frac{1}{2} \sum_{i \in \llbracket 1, d \rrbracket} \iint_{Q} s \gamma_{i} D_{i}\left(r^{2}\right) \tilde{\chi}^{i} D_{i}\left(u^{2}\right)=-\frac{1}{2} \sum_{i \in \llbracket 1, d \rrbracket} \iint_{Q} s D_{i}\left(\gamma_{i} D_{i}\left(r^{2}\right) \tilde{\chi}^{i}\right) u^{2} .
$$

With the results of Section 2.2, using that $D_{i} \gamma_{i}=\mathcal{O}(1)$ we find

$$
\left|\frac{1}{2} \sum_{i \in \llbracket 1, d \rrbracket} \iint_{Q} s \gamma_{i} D_{i}\left(r^{2}\right) \tilde{\chi}^{i} D_{i}\left(u^{2}\right)\right| \leqslant C\left\|s^{\frac{3}{2}} r u\right\|_{L^{2}(\omega)}^{2},
$$

for $h$ and $s h$ sufficiently small.

With (A.6)-(A.12) we finally obtain

$$
\begin{aligned}
& \sum_{i \in \llbracket 1, d \rrbracket}\left\|s^{\frac{1}{2}} r D_{i} u\right\|_{L^{2}\left((0, T) \times \omega_{0}\right)}^{2} \\
& \quad \leqslant C\left(\|r f\|_{L^{2}(Q)}^{2}+\left\|s^{\frac{3}{2}} r u\right\|_{L^{2}((0, T) \times \omega)}^{2}+\sum_{i \in \llbracket 1, d \rrbracket}\left\|r D_{i} u\right\|_{L^{2}(Q)}^{2}+h^{-1}\left|r u_{\mid t=0}\right|_{L^{2}(\Omega)}^{2}+h^{-1}\left|r u_{\mid t=T}\right|_{L^{2}(\Omega)}^{2}\right)
\end{aligned}
$$

which concludes the proof of Lemma 3.12 .

\section{References}

[1] V. Barbu, Exact controllability of the superlinear heat equation, Appl. Math. Optim. 42 (2000) 73-89.

[2] F. Boyer, F. Hubert, J. Le Rousseau, Discrete Carleman estimates and uniform controllability of semi-discrete parabolic equations, J. Math. Pures Appl. 93 (2010) 240-276.

[3] F. Boyer, F. Hubert, J. Le Rousseau, Discrete Carleman estimates for elliptic operators in arbitrary dimension and applications, SIAM J. Control Optim. 48 (2010) 5357-5397.

[4] F. Boyer, F. Hubert, J. Le Rousseau, Uniform null-controllability properties for space/time-discretized parabolic equations, Numer. Math. 118 (2011) 601-661.

[5] I. Bihari, A generalization of a lemma of bellman and its application to uniqueness problems of differential equations, Acta Math. Hung. 7 (1956) 81-94.

[6] F. Boyer, On the penalised HUM approach and its applications to the numerical approximation of null-controls for parabolic problems, preprint, http://hal.archives-ouvertes.fr/hal-00812964.

[7] T. Carleman, Sur une problème d'unicité pour les systèmes d'équations aux dérivées partielles à deux variables indépendantes, Ark. Mat. Astron. Fys. 26B (17) (1939) 1-9.

[8] T. Duyckaerts, X. Zhang, E. Zuazua, On the optimality of the observability inequalities for parabolic and hyperbolic systems with potentials, Ann. Inst. Henri Poincaré, Anal. Non Linéaire 25 (2008) 1-41.

[9] E. Fernández-Cara, S. Guerrero, Global Carleman inequalities for parabolic systems and application to controllability, SIAM J. Control Optim. 45 (4) (2006) 1395-1446.

[10] E. Fernández-Cara, A. Münch, Numerical null controllability of semi-linear 1D heat equations: Fixed point, least squares and Newton methods, Math. Control Relat. Fields 2 (3) (2012) 217-246.

[11] E. Fernández-Cara, E. Zuazua, Null and approximate controllability for weakly blowing up semilinear heat equations, Ann. Inst. Henri Poincaré, Anal. Non Linéaire 17 (2000) 583-616.

[12] A. Fursikov, O.Yu. Imanuvilov, Controllability of Evolution Equations, Lecture Notes, vol. 34, Seoul National University, Korea, 1996.

[13] L. Hörmander, On the uniqueness of the Cauchy problem, Math. Scand. 6 (1958) 213-225.

[14] L. Hörmander, Linear Partial Differential Operators, Springer-Verlag, Berlin, 1963.

[15] L. Hörmander, The Analysis of Linear Partial Differential Operators, vol. IV, Springer-Verlag, 1985.

[16] O.Yu. Imanuvilov, Controllability of parabolic equations, Mat. Sb. (N.S.) 186 (1995) 109-132 (in Russian).

[17] J. Le Rousseau, G. Lebeau, On Carleman estimates for elliptic and parabolic operators. Applications to unique continuation and control of parabolic equations, ESAIM Control Optim. Calc. Var. 18 (2012) 712-747.

[18] G. Lebeau, L. Robbiano, Contrôle exact de l'équation de la chaleur, Commun. Partial Differ. Equ. 20 (1995) 335-356.

[19] S. Labbé, E. Trélat, Uniform controllability of semidiscrete approximations of parabolic control systems, Syst. Control Lett. 55 (2006) 597-609.

[20] M. Yamamoto, Carleman estimates for parabolic equations and applications, Inverse Probl. 25 (2009) 123013.

[21] E. Zuazua, Control and numerical approximation of the wave and heat equations, in: International Congress of Mathematicians, III, Madrid, Spain, 2006, pp. 1389-1417.

[22] C. Zuily, Uniqueness and Non Uniqueness in the Cauchy Problem, Prog. Math., Birkhäuser, 1983. 